\title{
Modelling and Control of Auxiliary Loads in Heavy Vehicles
}

\author{
Niklas Pettersson
}

TRITA-S3-REG-0402

ISSN $1404-2150$

ISBN 91-7283-878-7

Automatic Control

Department of Signals, Sensors and Systems

Royal Institute of Technology (KTH)

Stockholm, Sweden, 2004

\footnotetext{
Submitted to the School of Electrical Engineering, Royal Institute of Technology, in partial fulfillment of the requirements for the degree of Technical Licentiate.
} 
Copyright (c) 2004 by Niklas Pettersson

Modelling and Control of Auxiliary Loads in Heavy Vehicles

Automatic Control

Department of Signals, Sensors and Systems

Royal Institute of Technology (KTH)

SE-100 44 Stockholm, Sweden

Tel. +4687906000

Fax. +4687907324

http://www.s3.kth.se 


\section{Abstract}

A possible method to save fuel in heavy vehicles is improved control of the auxiliary units. Today, the auxiliaries are often mechanically driven by the engine, and are thus constrained to revolute with a fixed ratio to the engine speed. This mechanical constraint results in energy losses. In the thesis, the benefits of driving the auxiliaries with electricity are evaluated. The output of an electrically driven auxiliary can at every time instant be controlled to match the actual need. Considered auxiliaries are electrical generator, water pump, cooling fan, air compressor, air conditioning compressor, oil pump and power steering pump.

The first part of the thesis presents an upper limit on what fuel saving that can be achieved if the auxiliaries are redesigned. The total energy consumption of the auxiliary units as they are designed today is estimated through computer simulations. The simulations indicate that the fuel consumption caused by the auxiliary units is in the range of $4.7 \%$ to $7.3 \%$ of the total consumption.

A Modelica library for simulation of the energy consumption of the auxiliary units is then presented. The library contains a mixture of models developed from physical principles and models fitted to collected data. Modelling of the cooling system is described in detail. Simulations of the cooling system show good agreement with measurements from wind tunnel tests.

A case study of optimal control of the cooling system is finally performed. Control actuators are the electrical generator, and the cooling fan and the water pump, which both are supposed to be electrically driven. The problem is posed as a constrained optimal control problem with feedforward from measurable external variables. The design is based on a simplified model derived from physical principles. It is evaluated through simulations with external variables collected from experiments. The results show that significant energy savings can be obtained. 



\section{Acknowledgments}

This thesis is conducted as a part of an industrial $\mathrm{PhD}$ project. I want to express my gratitude especially to Nils-Gunnar Vågstedt for getting opportunity to do this kind of studies being an employee at a company like Scania CV AB with the support it involves. You took a big initiative in starting the project and were my encouraging supervisor at Scania the first one and a half years.

Along all the times has been my supervisor at KTH, Dr. Karl Henrik Johansson. Great thanks for all guiding and encouraging in the research. When time came to work with papers and the thesis you gave priceless advice on writing clear and direct.

I want to express a great thank to my current supervisor at Scania, Michael Blackenfeldt. You took over the role as supervisor at Scania in a period when I felt that things were not going particular well. Michael showed understanding and support from the start and changed from being a colleague to become the boss and supervisor in an elegant way.

Many thanks to Prof. Bo Wahlberg for giving the opportunity to be a member in your department and for contributing with great experience in the steering committee of the project.

I want to give a special thank to Johan Lindström, Christer Ramden and Erik Söderberg at Scania who assisted with a lot of advice, contributed with measurements and took part in many productive discussions. Many thanks also to all other colleagues at Scania for fruitful discussions on issues not necessarily related to the project.

Great thanks to all people at S3-KTH! I always felt welcome; regardless if it went weeks between the times I showed up at the institute, or if at least I felt that I should change my address to Osquldasväg.

This work was partially sponsored by Programrådet för fordonsforskning $(\mathrm{PFF})$ which is gratefully acknowledged.

Finally, big thanks to all others, making the world a joyful place to be. 



\section{Contents}

1 Introduction 1

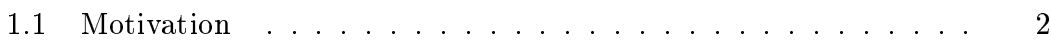

1.2 Related Work . . . . . . . . . . . . . . . . . 5

1.3 Outline and Contributions . . . . . . . . . . . . . 7

2 Energy Consumption of Auxiliary Units 9

2.1 Measures of Energy Consumption . . . . . . . . . . . . . . 10

2.2 Simulation Principle . . . . . . . . . . . . . . . . . . . . 12

2.3 Vehicle Specification . . . . . . . . . . . . . . . . . . 14

2.4 Drive Cycle . . . . . . . . . . . . . . . . . . . . . . 15

2.5 Simulation Models of Auxiliary Units . . . . . . . . . . . . 17

2.6 Simulation Results . . . . . . . . . . . . . . . . . 27

3 Modelling of Energy Consumption 33

3.1 Selection of Model Structure . . . . . . . . . . . . . . . . . . 34

3.2 Model Library . . . . . . . . . . . . . . . . . . . . . 37

3.3 Cooling System Module . . . . . . . . . . . . . . . . . . . . 38

3.4 Assembling the Total Model . . . . . . . . . . . . . . . . 50

3.5 Summary ....................... 54

4 Energy Optimal Control of the Cooling System 57

4.1 System Configuration .................. 59

4.2 Problem Formulation . . . . . . . . . . . . . . . . . . 63

4.3 Simulation Results . . . . . . . . . . . . . . . . . 70

4.4 Conclusions . . . . . . . . . . . . . . . . . . . 76

5 Summary and Future Work $\quad \mathbf{7 7}$

5.1 Summary . . . . . . . . . . . . . . . . . . 77

5.2 Future Work . . . . . . . . . . . . . . . 78

$\begin{array}{lr}\text { Bibliography } & 81\end{array}$ 



\section{Chapter 1}

\section{Introduction}

The fuel economy is one of the most important properties of heavy vehicles. This is especially true for long haulage trucks. In Western Europe, and regions with a similar economical situation, the cost of fuels represent approximately one third of the total cost for the owner of long haulage trucks. The truck owner is running a business where the success is depending on low cost, and is well aware of this fact. When times come to replace the old truck, brands with good fuel economy will be very competitive. The truck manufacturing companies of course respond to this demand and put efforts into lowering the fuel consumption wherever it is possible when developing new models and components. Naturally a good fuel economy must have its origin in a well tuned combustion process in the intrinsic engine. Substantial progress has been done over the years to increase the efficiency of the diesel engine used in heavy vehicles, and more remain to be achieved. Simultaneously all other losses in the vehicle have to be reduced. Examples of potential improvements are reduction of aerodynamic drag, rolling resistance and, the topic of this thesis, better utilisation of the energy consumed by auxiliary units such as pumps and fans driven by the engine.

The importance of auxiliary units with high efficiency is often stressed and a fair number of proposals on various novel designs are found in the literature, but very little of this can be seen in the vehicles on the market today. Well proven old designs are reused in new generations of vehicles. The auxiliaries are in general mechanically driven and effective means to control their output to the actual need are often lacking. Up to now, the energy saving possible to obtain with more advanced solutions could not 
make up for the increasing cost and complexity. However, as the price of fuel tends to increase, alternatives become more competitive. In the thesis the potential benefits of driving the auxiliary units electrically are analysed. Studied auxiliary units are

- Electrical generator.

- Cooling fan.

- Water pump.

- Air compressor.

- Air condition (AC) compressor.

- Oil pump.

- Power steering pump.

With electrical drives the output of the auxiliaries can be continuously adjusted to the desired level. Thereby the energy utilisation hopefully can be improved compare to a mechanical design with less control capability. However, the use of a common source of power also introduces additional interactions between the subsystems. In order to utilise the possibilities offered with the electrical drive and to handle the interactions in a sound way, the problem is analysed using systems and control theory. The thesis centres on this aspect while the actual design of components is not directly addressed.

\subsection{Motivation}

The application considered in the thesis is heavy vehicles used for long haulage transports. A typical vehicle combination for this category is depicted in figure 1.1. The towing vehicle is a tractor with totally four wheels of which two are driven, (so-called $4 \times 2$ ). The tractor in figure 1.1 is equipped with a 16 litre V8 diesel engine although the predominant choice of engines in this category is a 12 litre 6-cylinder. The three-axle box semitrailer is typical for general cargo transports in Europe where generally the gross weight of the combination is limited to 40 tons and the total length is restricted to 18 metres.

Long-haulage vehicles are more sensitive to fuel cost of then other types of heavy vehicles. Thus, spending efforts in developing more efficient systems will be most motivated for this type of vehicles. Even if 


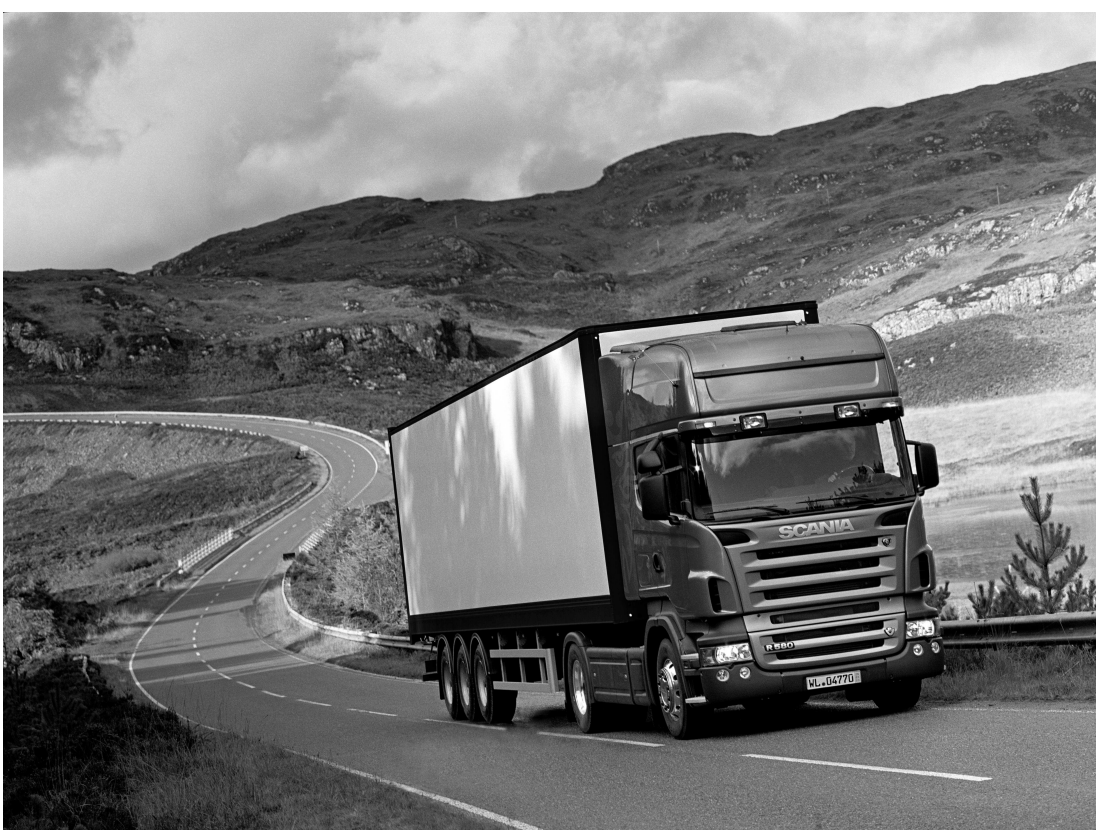

Figure 1.1: Scania R $5804 \times 2$ tractor with a three axle semitrailer driving a country road in Great Britain.

the development may lead to more complex and expensive solutions, it might be motivated if the fuel saving is big enough. Furthermore, many of the truck manufacturers have their bulk of the production volume in the long-haulage category. The performance of the product line of the manufacturer is often optimised for the long-haulage trucks. That design is then reused with minor modification for other types of heavy vehicles like for instance construction trucks. Thus, efforts spent on improving the performance of the long-haulage trucks will affect also other categories of heavy vehicles.

A key factor that makes it interesting to consider electrification of previously mechanically driven devices and redesigns of the electrical system in heavy vehicles is the recent development of hybrid electrical passenger cars. The hybrid technology can be an enabler for passenger cars, city busses and light-weight trucks with less environmental impact and increased performance. However, for heavy trucks and especially in long 
haulage transports, however, the technology seems to be less suitable. Compared with other vehicles, the engine in long-haulage trucks typically operates in a narrower speed range and at a power output closer the maximum capacity. Therefore the average efficiency of the engine over the drive cycle comes closer to the maximum than in other vehicle applications, and thus, the potential improvement with hybridisation is lower. Nevertheless, the technology development can be of use even here, since power electronic components with specifications and pricing adjusted for automotive use are likely to be available on the market in a few years. Components intended for propulsion of passenger cars may with smaller modification very well be suitable for other tasks in heavy vehicles. The same reasoning is valid also for the design methodology of the hybrid vehicles: for instance much of the considerations about control principles for hybrid vehicles are applicable in the control of electrical power in heavy vehicles.

Some prototypes of electrically driven auxiliaries intended for heavy duty vehicles can be found already today. For example, the company Engineered Machined Products (EMP), has designed electrical water and oil pumps. Products from EMP are presented in Petterssons case study on controllable cool-water flow [Pet02], in the overview of parasitic losses by Hnatczuk et al. [HLBG00], and in the case study on an electrical oil pump presented by Lasecki and Cousineau [LC03].

While the demand on lowering the fuel consumption gives a willingness to employ new technologies, the even more stressed requirement on dependability for heavy vehicles acts in the other direction and cause a resistance against new components. If a significant change of a design is done, possibly years of development and thorough tests have to be performed before introducing the novelty on the market. Before making decisions to take such a step, a broad evaluation must be conducted where the potential benefits with the new technology are assessed. The thesis tries to play the role of being this kind of feasibility study. The evaluation relies on theoretical reasoning and simulations where the objective is to prepare for a decision on if more practical and thereby more costly development activities should be started. In order to give the theoretical work a practical value, as much knowledge as possible of real-world operating conditions is put into the study. 


\subsection{Related Work}

Research related to modelling and control of auxiliary units in heavy vehicles is presented both in publications specified on automotive applications and in more general publications in the field of automatic control. An ambitious plan for reducing fuel consumption and emission for trucks in general on the North American market is presented in the Technology Roadmap for the 21st Century Truck Program [DOE00]. This is a declaration of a joint governmental and industrial research program which covers trucks from approximately four tons up to heavy long-haulage trucks (class 8 ). Within the program, increasing the efficiency of the auxiliary units in long haulage trucks is pointed out as one of the prioritised areas.

In chapter 2, an overview of the auxiliary units is given and their individual energy consumption is assessed through simulations. Similar work is conducted by Hendricks and O'Keefy in [HO02]. The result presented in [HO02] however refer to trucks in North America. The conditions in North America differ rather substantial from the conditions in Europe. For instance different regulations on weight and size of heavy vehicles result in a quite dissimilar design of the vehicle itself, while different regulations on speed limits and working conditions for the drivers result in a dissimilar usage. The corresponding result presented in chapter 2 is derived with the objective to give a picture of the situation in Western Europe in general and applies in particular to Scania vehicles. Models of auxiliary loads in heavy vehicles intended for the Simulink-based simulation program ADVISOR $\left[\mathrm{BHH}^{+} 02\right]$ are derived in the work by O'Keefy et al. [OHLB02]. The level of detail of these models spans from simple look-up tables representing the power consumption of the air compressor up to a complex simulation model of the climate system. Yet again, this work refers to conditions in North America and is not possible to directly convert to European conditions although similarities undoubtedly exist.

The model library presented in chapter 3 fits into a long term activity of developing complete vehicle models at Scania. It takes its starting point in the work by Sandberg [San01], who developed models which can be used to evaluate powertrain configurations. The latest activity in this direction at Scania is the work by Bengtsson [Ben04], where the need for a general model library is surveyed and an initiative to further structure the current model library is presented. Obviously other automotive companies have the same need for developing proprietary model libraries to be used in various tasks like for instance assessing impacts on fuel consumption. In [TBD03] Tiller gives a presentation of 
a Modelica library developed at Ford Motor Company. Similar academic attempts include the framework for evaluating hybrid electrical vehicles presented by Laine and Andreasson [LA03]. The objective in [LA03]is to build a general-vehicle library in Modelica which can be used in studies of different aspects of hybrid configurations.

In chapter 4, a case study on energy optimal control of the cooling system is presented. Deriving an energy efficient control of auxiliary units with the possibility to buffer energy in for instance a battery, clearly relates to the task of controlling hybrid electrical vehicles. This type of problem naturally fits into the framework of optimal control theory. Minimising fuel consumption is a logical choice of optimisation criterion and constraints on input and state variables can be incorporated in the theory. The framework of optimal control theory has been developed since the 1950's. A textbooks in the area is [BH75]. In chapter 4, prediction of future external influences to the system is utilised to facilitate the control. Similar approaches are often discussed in the control of hybrid electrical cars. For instance, a cluster consisting of researcher from Daimler Chrysler and the University of Karlsruhe has contributed with several studies in this area, e.g., [BTK04]. The prediction of the future influences is considered to be realised with aid of an onboard GPS receiver and digitalised maps. Interesting result on the implementation of such schemes is presented in [FB04]. A well known challenge is to implement schemes based on optimal control theory in the cheap hardware control units available in vehicles. The computational power of the control units increases rather rapidly though and possibly this concern might just be of historical interest in a few years. On the other hand, complexity of the algorithms is always an important issue regardless of the computational power available. It is desirable to implement control schemes in a compact and straight forward manner in order to increase understanding of the controller behaviour, and facilitate portability and maintenance of software components. Therefore it is advantageous to reduce the complexity of algorithms regardless of the computational power available and consequently approximate solution methods are of great interest. The thesis do not deal with the possibility of actual implementation of the algorithms developed in chapter 4 . Some discussion on possible ways to move towards a realisation is discussed in the outline of future work in chapter 5 . The Equivalent Consumption Minimisation Control (ECMS) is an interesting method to derive controllers for parallel hybrid electrical vehicles, see e.g. [SBG04]. The control strategy presented in [SBG04] approaches the optimal performance and is feasible for real-time execution on currently 
available hardware. Another pathway to follow is to somehow approximate the model of the system dynamics. The system model can for instance be approximated with a time discrete and piecewise affine system, see e.g. [BABH00]. Then techniques derived for predictive control of linear system can be applied. A survey of predictive control is given in [Mac02]. Further, it has been shown that for linear and piecewise affine systems it is possible to find an explicit control law which can be computed in advance off-line [BMDP02]. Thus there is several optional ways to derive control schemes with performance close to optimal which can be executed in real-time. Yet it is valuable to find the true optimal solution even if it is not implemented. Firstly the optimal solution gives a quantification of what the achievable performance is. Secondly the process of finding the optimal solution gives insight in the nature of the problem which can be used when designing a good-enough controller that can be realised.

\subsection{Outline and Contributions}

The thesis starts with an introductory survey in chapter 2 over the auxiliary units as they are designed today and how they influence the overall fuel economy. The consumption of the individual subsystems is derived through simulations. In chapter 3 , work with models for simulation of the energy consumption of the auxiliary units are presented. Particularly the development of a Modelica library and modelling of the cooling system are discussed. The work in chapter 3 has been presented in:

N. Pettersson and K. H. Johannsson. Simulating energy consumption of auxiliary units in heavy vehicles. In Proceedings of 13th IFAC Symposium on System Identification, 2003.

and

N. Pettersson and K. H. Johannsson. Modelica library for simulating energy consumption of auxiliary units in heavy vehicles. In Proceedings of the 3rd International Modelica Conference, 2003. 
Chapter 4 presents a case study on energy optimal control of the cooling and the electrical system. Optimal control theory is employed to derive the control of an electrically driven water pump and cooling fan, and the control of the generator. The work in chapter 4 has presented in:

N. Pettersson and K. H. Johannsson. Optimal control of the cooling system in heavy vehicles. In Proceedings of 1 st IFAC Symposium on Advances in Automotive Control, 2004.

and

N. Pettersson and K. H. Johannsson. Optimal control of the cooling system in heavy vehicles. In Reglermötet, 2004.

Finally in the last chapter the results are summarised and proposals for future work are given. 


\section{Chapter 2}

\section{Energy Consumption of Auxiliary Units}

This chapter aims at giving an overview of the auxiliary units and there influence on the overall fuel consumption for long haulage vehicles. Further, the ambition is to give an apprehension of the relative significance of the individual auxiliaries in this sense. Hopefully the results can work as a guideline on where to put effort on new designs in order to decrease the fuel consumption and give estimates of what the potential of such efforts is. Figure 2.1 summarises the maximum and the minimum power consumption of the auxiliaries in a modern Scania truck at $1400 \mathrm{rpm}$ engine speed. The power consumptions shown in figure 2.1 take into account the full range in which the operation can vary (at a fixed engine speed). For the water pump and the oil pump, the power consumption is constant at a constant engine speed. For the other auxiliaries, the consumption depends on several other quantities. In this chapter we try to estimate through computer simulations what their influence are on the fuel consumption during a typical drive cycle. In order to do this, measures of the fuel consumption of the auxiliary units is defined in section 2.1. Thereafter the employed simulation principle is described in section 2.2. The specification of the vehicle and the drive cycles applied in the evaluation are presented in section 2.3 and section 2.4 , respectively. In section 2.5 , the simulation models of the auxiliary units are presented. Finally in section 2.6 the result of the simulation presented and conclusions from the evaluation are discussed. 


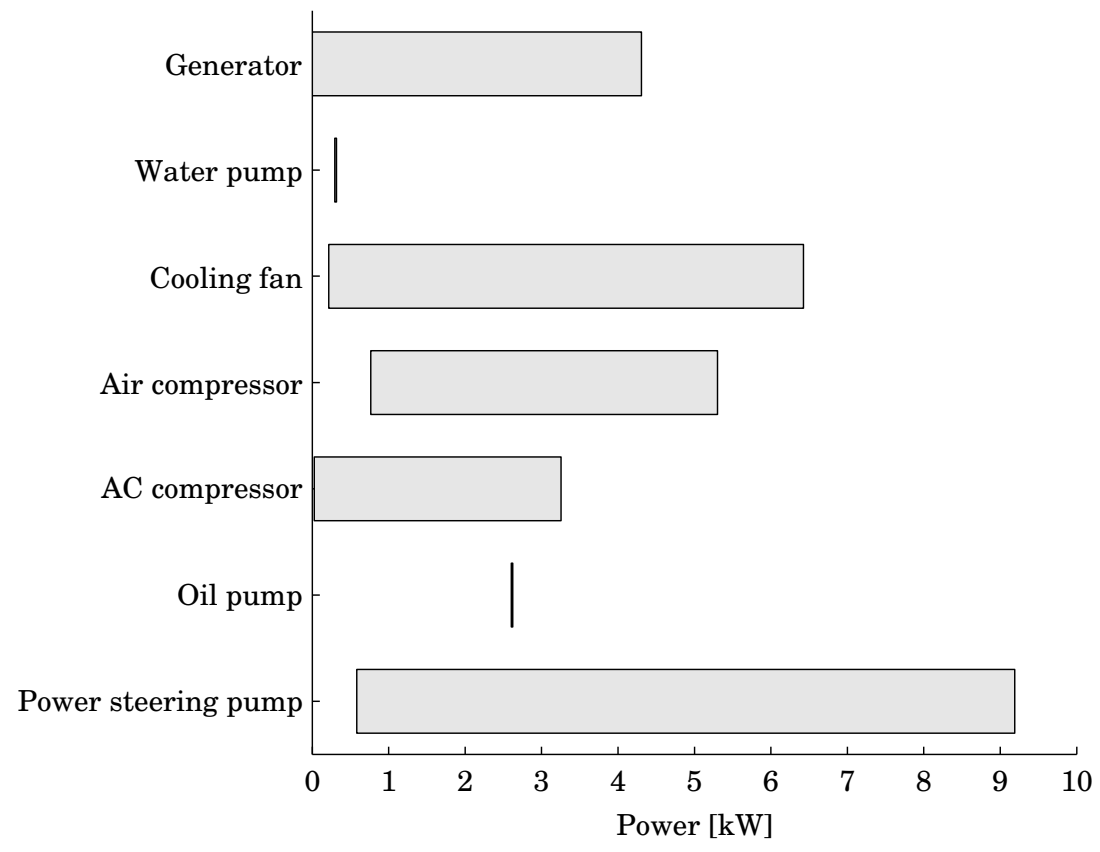

Figure 2.1: Summary of the auxiliary units with their minimum and maximum power consumption when operated at an engine speed equal to $1400 \mathrm{rpm}$.

\subsection{Measures of Energy Consumption}

The overview of the auxiliaries shown in figure 2.1 illustrates the range of possible power consumptions but does not tell what the consumptions are under normal operation. A more specific measure is the mean power consumption for a given drive cycle. If $T$ is the duration of the drive cycle and $P_{\text {aux }}(t)$ is the instant power taken from the engine to drive a certain auxiliary, the mean power consumption is

$$
\bar{P}_{\mathrm{aux}} \triangleq \frac{1}{T} \int_{0}^{T} P_{\mathrm{aux}}(t) d t
$$

This measure gives a good view of the actual consumption but cannot be directly translated to how much the auxiliaries influence the overall 
fuel consumption. The correlation in time between the auxiliary and the engine load will influence how much the drive of the auxiliary units cost in terms of fuel consumption. Even if two auxiliary units have equal mean power consumptions they do not necessarily influence the overall fuel consumption equally. For instance, assume that the first auxiliary unit have constant power consumption while the second have higher consumption when the vehicle is braking and lower when the vehicle is cruising in constant speed. Then the operation of the first auxiliary will result in higher fuel consumption than the operation of the second. The reason for this is that second auxiliary utilise more of the free energy that is available when the vehicle is braking. In order to asses how the overall fuel economy is influenced, the fuel consumption of a vehicle equipped with the specific auxiliary unit when driving a certain route may be compared with the fuel used to drive the same route with the auxiliary unit removed. The difference in consumption between the case with and without the auxiliary unit can be expressed in fuel consumption per travelled distance or time. Here the mean fuel consumption per travelled distance is chosen as measure. Let $F_{\text {aux }}$ and $F_{0}$ denote the amount of fuel spent when driving with and without the auxiliary, respectively, and $d$ the total distance travelled in the drive cycle. Then the mean fuel consumption for the auxiliary per travelled distance is:

$$
\bar{f}_{\text {aux }} \triangleq \frac{F_{a u x}-F_{0}}{d}
$$

For heavy vehicles, more than for passenger cars, the absolute fuel consumption differs rather much from one driving situation to another. This means that it might be hard to value the average fuel consumption in (2.2) expressed in for instance litres per $100 \mathrm{~km}$. A measure relating the consumption of the auxiliaries to the total fuel consumption is easier to apprehend. The following relative measure for the auxiliary part of the fuel consumption is therefore proposed:

$$
r_{\mathrm{aux}} \triangleq \frac{F_{\mathrm{aux}}-F_{0}}{F_{0}}
$$

In this chapter the mean power consumption, $\bar{P}_{\text {aux }}$, the mean fuel consumption, $\bar{f}_{\text {aux }}$ and the relative part of the fuel consumption, $r_{\text {aux }}$, are derived for the auxiliary units summarised in figure 2.1. The measures are dependent of the vehicle specification and the driving situation. In order to answer the right questions, concern has to taken when selecting these. In this study, the most common type of heavy vehicle used for long 


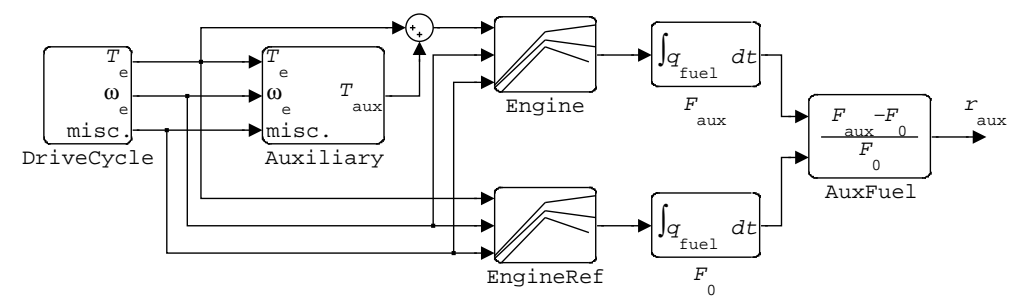

Figure 2.2: Block diagram of the principle used to calculate the fuel consumption of the auxiliary units.

haulage transportation of goods in Western Europe is analysed through simulations. The driving situation is chosen to be representative for this class of vehicles.

\subsection{Simulation Principle}

The simulation principal used for deriving the power consumption of the auxiliary units is illustrated in the block diagram in figure 2.2. Models of the auxiliary units are developed in Simulink. In figure 2.2 the block labelled Auxiliary represents one instance of an auxiliary model. Inputs to the auxiliary block are time trajectories describing the operation of the vehicle. These are contained in the block labelled DriveCycle and are obtained from simulations of a complete vehicle model performed in advance. The primary output from the models of the auxiliary units is the additional torque on the driveline, denoted $T_{a u x}$, which is needed to run the auxiliaries. The sum of the torque for the auxiliary unit and the original engine torque is fed to the inverse model of the engine labelled Engine. Here the amount of fuel flow needed to create the total torque is calculated. Further, the original engine torque is fed to a reference engine labelled EngineRef in order to calculate the amount of fuel that would be consumed without the auxiliary load. The outputs of the engine models are integrated with respect to time and fed to the block labelled AuxFuel where the auxiliary part of the fuel consumption is calculated according to equation (2.3).

The technique to calculate the input that would yield a certain predefined state trajectory is sometimes referred to as an inverse or backward 
simulation in contrast to standard forward simulations where the state trajectory is regarded as the output. Here forward and backward simulations are combined. Trajectories of the engine speed and torque are used to obtain the required input fuel flow in a backward manner while the internal states in the auxiliary model are simulated in a forward manner. A similar simulation principle is used in Advisor [WCB99].

In the simulation, the time trajectory of the vehicle speed, engine speed, the total torque on the drive wheels etc. is regarded as independent of the auxiliary units. The input trajectories are obtained by initially running a simulation with a Modelica model used at Scania to evaluate the fuel consumption for complete vehicles [San01]. In the initial simulation, the vehicle is set to drive a road with varying topology and speed limit that have been obtained from recordings of real roads. The vehicle is assumed to run on cruise control and with computer-controlled gear shifting (automated manual transmission). When going downhill, automated braking is performed with the secondary hydraulic brake called the retarder. Algorithms from the production version of these control schemes are incorporated in the simulation model. The vehicle model has been validated with respect to the energy consumption of the combustion engine and losses such as rolling resistance and air drag. In the initial simulation, influences from the auxiliary units are included as a lumped net power added to the driveline torque. Before the output of the complete vehicle model is used as input to the simulations presented here, this lumped auxiliary power is removed.

The assumption that the auxiliary units do not influence the overall vehicle trajectories is a simplification. If the auxiliary load is large enough, it might cause a change in the gear shifting strategy. Further, when the vehicle is cruising downhill with no torque provided from neither the engine nor the retarder, the additional load from the auxiliaries will decrease the speed. These effects are assumed to be small since the auxiliary power is small in comparison to the total driveline power.

The models of the individual auxiliary units are described in section 2.5. The inverse models of the engine, which are used to calculate the amount of fuel needed to create a certain torque, are based on tables obtained from measurements in an engine test cell. The tables describe the operation of the engine in steady state and do not include transient behaviour such as for instance time lag in the turbo. However, in high speed driving these transient effects have minor effect on the overall fuel consumption and neglecting them in this study will likely have no significant influence. The total torque is calculated as the sum of the drive 


\begin{tabular}{|c|c|c|}
\hline Component & Characteristic & Type designation \\
\hline Vehicle & $\begin{array}{l}\text { Tractor-semitrailer. } \\
40 \text { tons G.W. } \\
4 \times 2 \text { tractor. } \\
\text { Three-axle semitrailer. } \\
\text { Frontal area } 10.36 \mathrm{~m}^{2} . \\
\text { Coefficient of air drag } 0.56 \text {. }\end{array}$ & Scania $124 \mathrm{~L}$ \\
\hline Engine & $\begin{array}{l}12 \text { litre inline } 6 . \\
420 \text { Hp. } \\
\text { Euro IIIb emission standard. }\end{array}$ & DC1202RB \\
\hline Gear box & $\begin{array}{l}\text { Automated manual } \\
\text { with retarder. } \\
16 \text { speeds }\end{array}$ & GRS900R \\
\hline Final gear & Ratio 3.08 & R780 \\
\hline Front tiers & $\begin{array}{l}\text { Rolling resistance } 5.3 \mathrm{~kg} / \text { ton. } \\
\text { Rolling radius } 523.6 \mathrm{~mm} .\end{array}$ & $315 / 80$ R22.5 XZA1 \\
\hline Rear tiers & $\begin{array}{l}\text { Rolling resistance } 7.0 \mathrm{~kg} / \text { ton. } \\
\text { Rolling radius } 528.4 \mathrm{~mm}\end{array}$ & $315 / 80$ R22.5 XDA \\
\hline Trailer tiers & $\begin{array}{l}\text { Rolling resistance } 5.9 \mathrm{~kg} / \text { ton. } \\
\text { Rolling radius } 523.6 \mathrm{~mm} .\end{array}$ & $315 / 80$ R22.5 XZE2 \\
\hline $\begin{array}{l}\text { Ambient } \\
\text { conditions }\end{array}$ & $\begin{array}{l}\text { Air temperature } 20^{\circ} \mathrm{C} \text {. } \\
\text { Air pressure } 101.3 \mathrm{kPa} \text {. }\end{array}$ & \\
\hline
\end{tabular}

Table 2.1: Summary of vehicle characteristics used in the analysis.

line torque and the torque needed for the auxiliaries. In situations when the engine is operated at its maximum capacity, the required total torque might exceed the engine capacity when adding the auxiliary load. Then the tables are extrapolated so that the amount of fuel required for the total torque corresponds to what would have been consumed by a slightly larger engine.

\subsection{Vehicle Specification}

In table 2.1 the specification of the chosen vehicle configuration is summarised. The vehicle chosen in this survey is a tractor semi-trailer combination with a 12 litre $420 \mathrm{hp}$ engine and a gross weight of 40 tons. This combination is the most common for long haulage transports in Europe and is often used in benchmarking tests. 

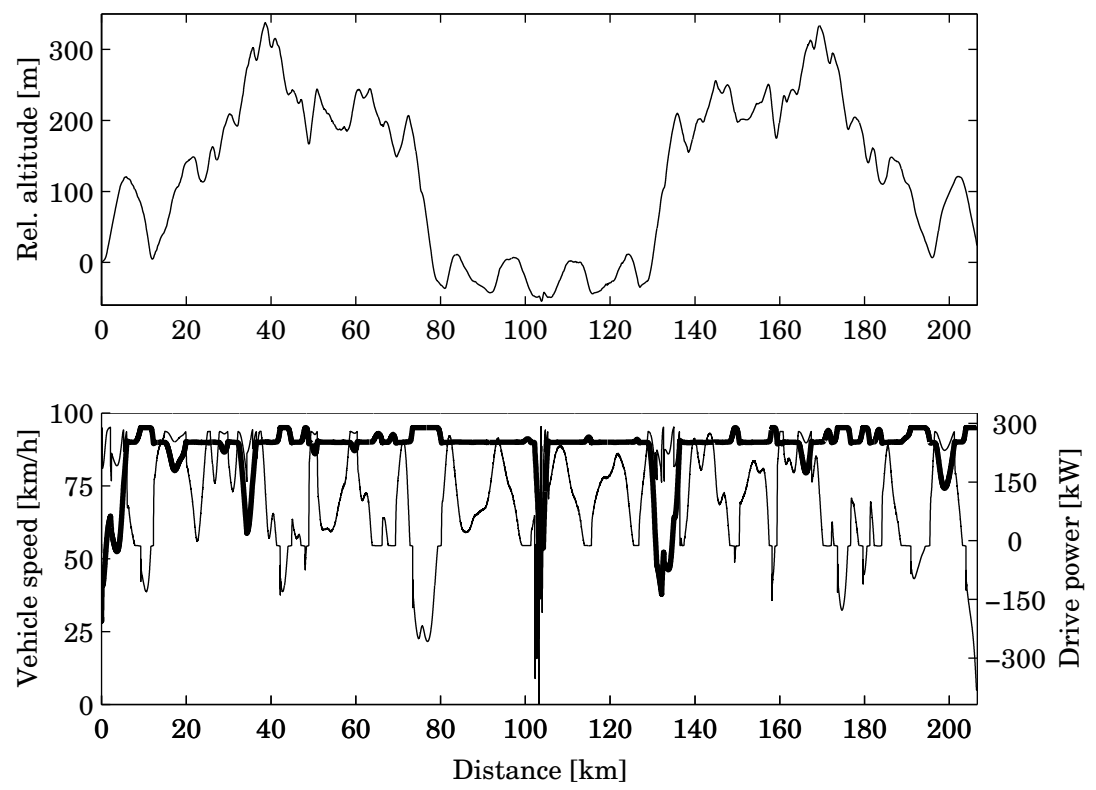

Figure 2.3: Road altitude (upper), and vehicle speed (thick lower) and drive power (thin lower) on the Koblenz-Trier route.

\subsection{Drive Cycle}

The drive cycles used in the analysis correspond to two specific roads, which are representative for long haulage traffic in Europe. Consequently they primarily consist of highway driving. The reference vehicle speed is at most parts of the cycles restricted by the set point of the electronic speed limiter which is compulsory for this category of heavy vehicles within the European Union. The technical speed limit of the electronic device is defined to not exceed $89 \mathrm{~km} / \mathrm{h}$. It is assumed that the vehicle hold this speed on road sections where the legal limit for passenger cars is above the technical limit $89 \mathrm{~km} / \mathrm{h}$, even though the maximum allowed speed for articulated heavy vehicles is $80 \mathrm{~km} / \mathrm{h}$. In some sections of the roads, the legal limit is lower or it is impossible to hold the maximum allowed speed with the vehicle combination due to for instance the inclination or the curvature of the road. When going downhill and the retarder 

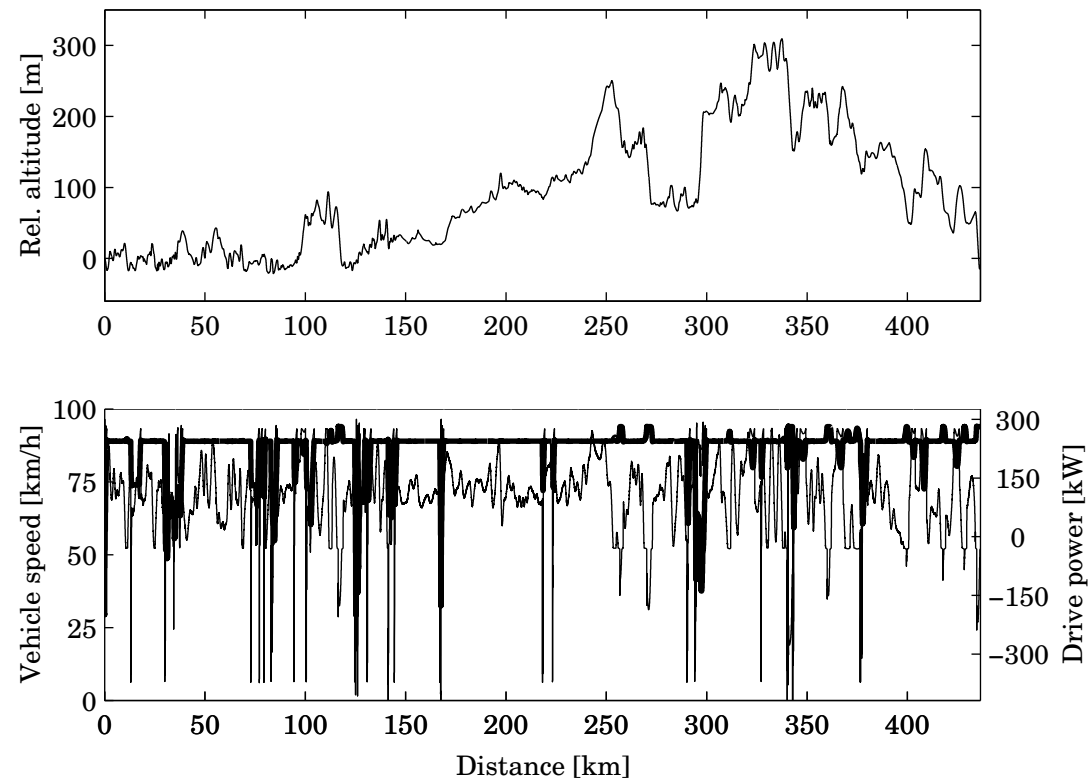

Figure 2.4: Road altitude (upper), and vehicle speed (thick lower) and drive power (thin lower) on the Södertälje-Gothenburg route.

is used to control the speed, the reference speed is set to $6 \mathrm{~km} / \mathrm{h}$ higher than the active limit when the speed is controlled with the cruise control acting on the engine. The speed limitations and the road inclinations used in the simulation are obtained from a data base of recorded road profiles available at Scania.

The first road section goes between the cities Koblenz and Trier in Germany. The trip starts in Koblenz and turn back to the starting point when reached Trier. The second road runs between the cities Södertälje and Gothenburg in Sweden. It starts in Södertälje passes Jönköping and ends in Gothenburg.

The selected routes are often used within the Scania development for reference driving. They differ from each other in the variation of road inclination. The first route contains some rather hilly sections mixed with more flat sections. The second route is more even in the distribution of road inclinations. As a result of the difference in the character of the 

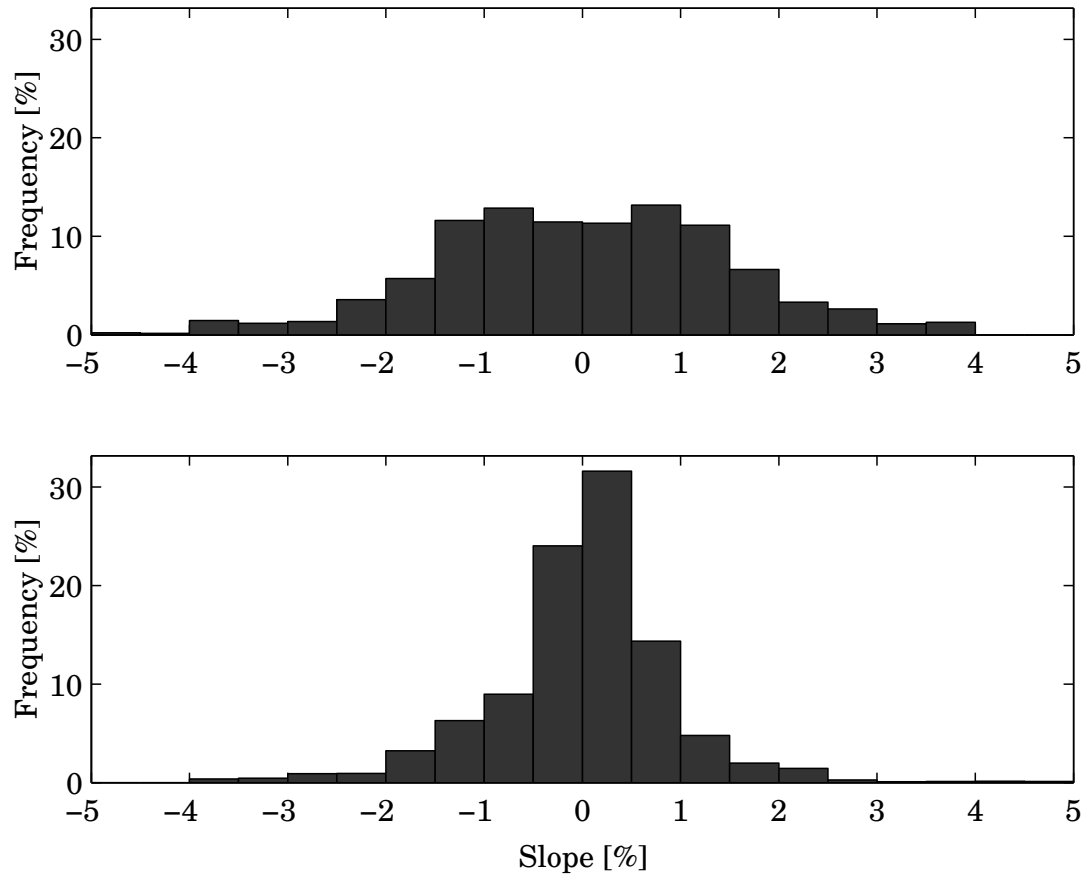

Figure 2.5: Distribution of road slopes on the Koblenz-Trier route (upper), and on the Södertälje-Gothenburg route (lower).

road topology, the total fuel consumption is higher on the German road than on the Swedish one. In figures 2.3 and 2.4 the vehicle speed, the road altitude and the required drive power of the drive cycles are shown. Figure 2.5 shows the statistical distribution of the road inclination on the two roads.

\subsection{Simulation Models of Auxiliary Units}

Individual simulation models of the auxiliary units are developed in order to study their influence on the overall fuel consumption. The prime objective is to accurately model the energy consumption. To do so, more or less involved models may be needed. The required level of detail depends 
on the possibility to control the output of the auxiliaries. With small or no possibility to control a certain auxiliary, a simple model may suffice since the operation could directly be described by the drive cycle of the vehicle itself. For instance, this is the case with the oil pump, where the operation is directly given from the operation of the engine. If more control can be applied to a specific auxiliary, a more detailed model may be required in order to simulate all the inputs the control depend on. The latter case applies to the cooling system, where the control depends on several variables which thus have to be included in the model. Generally the auxiliary units may exist in several versions. Where it is justified here, these variants are simulated and compared.

\section{Electrical generator}

Electricity in heavy vehicles is used for instance for illumination, for various comfort equipments, such as radio and coffee makers, and for powering electronic control units. The electrical power is produced by a beltdriven generator. The generator is a so called claw pole machine, which is the same type of generator as in passenger cars. When the engine is not running, or when the capacity of the generator is insufficient, electrical power is provided by the battery. The voltage level in the electrical system of heavy vehicles is $24 \mathrm{~V}$ in most regions, with North America as the main exception where a $12 \mathrm{~V}$ system is used. The generator is regulated to achieve a constant voltage level of approximately $28 \mathrm{~V}$ which is the charging voltage of the lead acid batteries.

In the simulation model, the power output of the generator is assumed to be exactly equal to the power consumption. Thus, no dynamics of the battery is considered. The generator is modelled as an energy transformation from mechanical to electrical energy with a constant efficiency factor. Further, the electrical output is assumed to be constant throughout the drive cycle. In reality the electrical output varies substantially between different vehicles, and with time on a specific vehicle. The variations depend on the amount of electrical devices installed and on operating conditions such as the ambient light and temperature. The constant output level in the simulation is set to $1120 \mathrm{~W}$ (corresponding to $40 \mathrm{~A}$ at $28 \mathrm{~V}$ ). This level is considered to be a reasonable average for modern trucks operating in Western Europe. 
According to the above assumptions, the complete model of the electrical system becomes

$$
P_{\mathrm{el}}=\eta_{g} P_{\mathrm{mech}}
$$

where $P_{\mathrm{el}}$ is the constant electrical output, $P_{\mathrm{mech}}$ is the resulting mechanical load on the engine from the generator, and $\eta_{g}$ is the constant efficiency of the generator. The efficiency of the generator is assumed to be $65 \%$, i.e., $\eta_{g}=0.65$.

\section{Cooling fan and water pump}

The principal layout of the cooling system in a Scania truck is illustrated in figure 2.6. The pump drives the flow of coolant fluid through the engine and the retarder. The combustion process in the engine generates heat, which is transmitted to the coolant. The retarder is a hydraulic brake mounted on the secondary side of the gearbox. When used, it releases heat into the cooling system. The heat transferred to the coolant is emitted in the air-cooled radiator. The air enters at the air intake at the front of the truck cab and exits at the air outlet at the rear. The airflow is partly driven by the fan and partly by the pressure build up caused by the wind speed at the intake and outlet. The air is used to cool both the radiator and the turbo charged intake air to the engine. The charge air cooler and the radiator are connected in series, see figure 2.6.

The model of the cooling system contains seven dynamic states. Five states represent the temperature in different parts of the cooling system: the temperatures of the coolant in the engine, in the retarder and in the radiator, and the temperatures of the engine block and the retarder oil. The remaining two states represent the dynamics of the thermostat and the fan clutch. In chapter 3, a Modelica model containing a fairly detailed description of the cooling system is presented. The model used in this study is a simplification of the Modelica model. Figure 2.7 shows that the output of the simplified model agrees well with the Modelica model and measurements from a wind tunnel.

The auxiliary units in the cooling system that consume energy are the water pump and the fan. The pump is belt driven so the speed of the pump is proportional to the engine speed. The total coolant flow from the pump is roughly proportional to the speed, while the flow through the radiator is regulated by the thermostat. The fan is connected to the engine crankshaft via a viscous clutch, which enables a passive speed control. The speed of the fan can be controlled up to a speed close to the engine speed. When the clutch is completely filled with oil, the speed of 


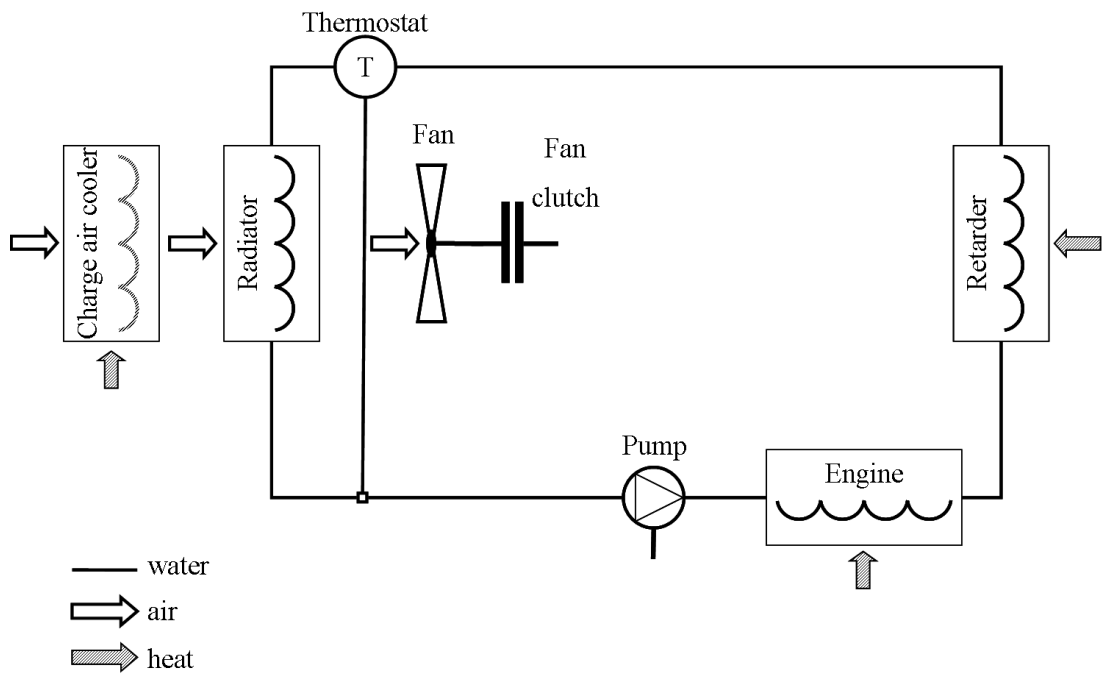

Figure 2.6: Principle layout of the cooling system.

the fan will approach the engine speed although there always will be a remaining slip speed of about $3 \%$ of the engine speed. The lowest possible speed of the fan, the idle speed, is restricted by the minimum torque that is transferred when the oil is evacuated in the clutch. For Scania vehicles, two variants of the fan clutch exist. In the older version, the operation of the clutch is controlled via a bimetal valve inside the clutch, while in the newer version, the clutch is electronically controlled. Besides the different control principles, the variants differ in the idle speed. The idle speed in the older version is in the range 600 to $700 \mathrm{rpm}$, while the idle speed in the never version typically is in the range 200 to $300 \mathrm{rpm}$.

The bimetal in the old version reacts on the temperature of the surrounding air and is thus acting as a feedback control of the overall cooling temperature. In the newer version, the clutch operation is controlled from the engine control unit. A block diagram of the electronic fan control is shown in figure 2.8. The control of the fan clutch is based on feedback from coolant temperature, charge air temperature, engine speed and vehicle speed. In addition to this, the fan clutch is controlled based on external request from other control units in the vehicle. In the simulation model, external request from the retarder control unit is incorporated. 


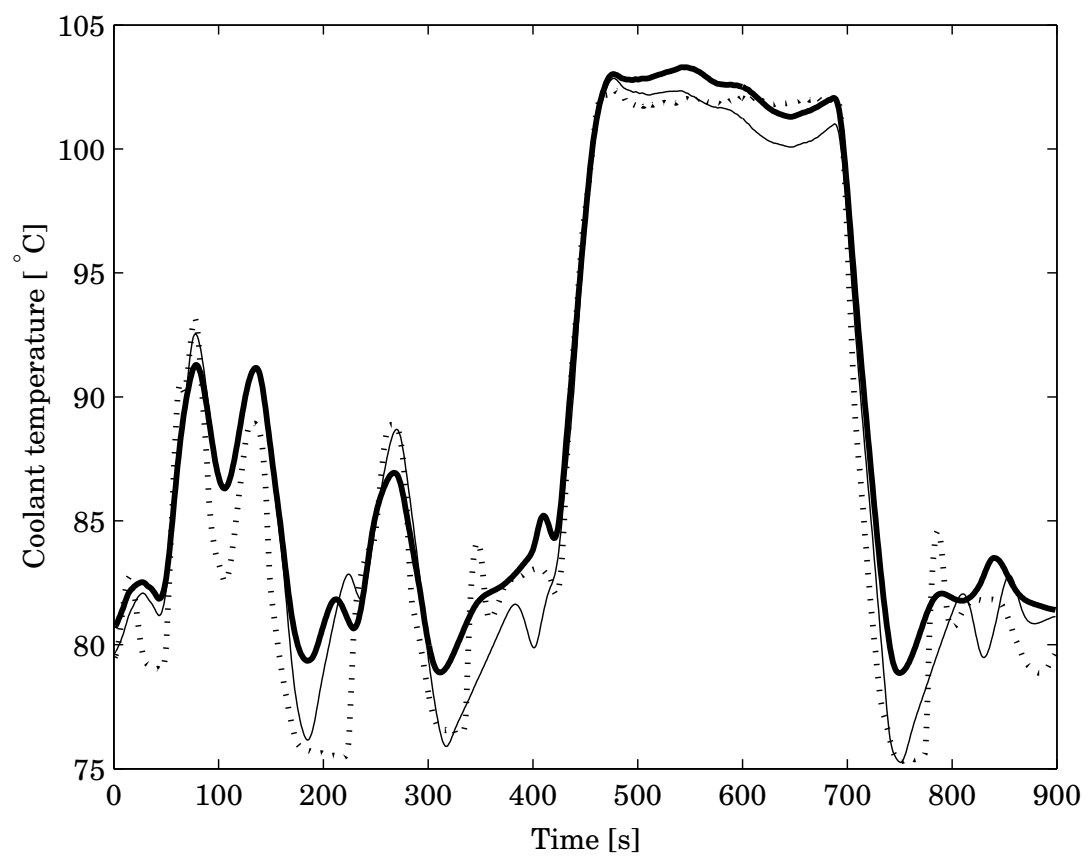

Figure 2.7: Coolant temperature obtained with the model used for the evaluation here (thick solid) compared with temperature obtained with the Modelica model (thin solid) and with measurements (dotted).

The retarder control unit apply control of the fan based on feedback from the coolant temperature, retarder torque, propeller shaft speed and ambient temperature. The source code of these algorithms is included in the model.

The operation of the fan with the old version of the clutch depends strongly on the dynamics of the oil and the bimetal inside the clutch. No detailed modelling of that dynamics is done. Instead, the electronic control is applied also in the simulation of the old clutch. In order to mimic the behaviour of the old clutch, the idle speed is set to $650 \mathrm{rpm}$ instead of $250 \mathrm{rpm}$ which is used when simulating the new clutch. 


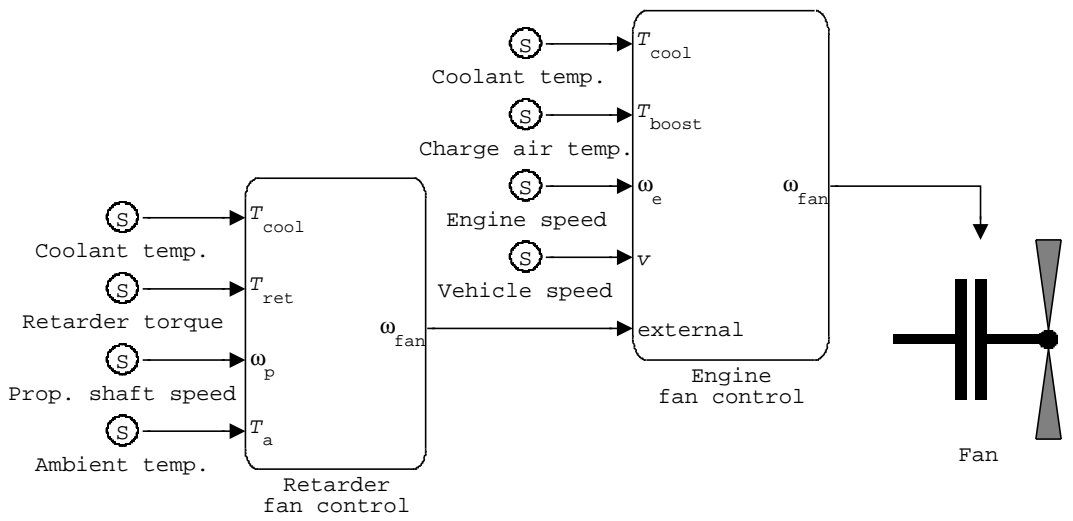

Figure 2.8: Structure of the fan control.

\section{Air compressor}

In heavy vehicles, compressed air is used to actuate the pneumatic brakes and various pneumatic valves. The air is compressed with a piston compressor which is driven by the engine via a gear transmission. The compressed air is stored in tanks mounted on the vehicle chassis. When filled, the pressure inside the tanks is approximately 8-10 bars.

In the simulation model, the compressed air system is modelled as a single volume representing the tanks with compressed air. It is assumed that the volume exhibit isothermal conditions. The motivation for this assumption is that the air tanks on the truck are purely thermally insulated so that the compressed air will cool down to a constant level relative to the ambient temperature. Thus, most of the energy that is converted to heat when the pressure is increased by the compressor is dissipated to the ambient. Under this assumption the remaining dynamic state of the compressed air system will be the pressure inside the air volume. The state equation for the pressure derived from the ideal gas law assuming constant temperature becomes

$$
\dot{p}=\frac{p\left(q_{\text {in }}-q_{\text {out }}\right)}{V}
$$

Here $q_{\text {in }}$ and $q_{\text {out }}$ denote the volume flow of delivered air from the compressor and the volume flow of consumed air, respectively. The volume, $V$, 
in equation (2.5) is estimated from the time it takes to fill the air tanks on the truck. The pressure in the air system is controlled inside a hysteresis band by switching the compressor between the active state and the inactive state. In the active state, air is compressed and delivered to the air system while in the inactive state no compressed air is produced. When the compressor is in the active state, the power consumption and the delivered air depend on the compressor speed and the pressure in the air tanks. In the inactive state, the power consumption solely depends on the speed. In the simulation model the power consumption and delivered air is calculated from look-up tables. The relation between the duration of the active state, $t_{\text {active }}$, and the duration of the inactive state, $t_{\text {inactive }}$, is referred to as the duty cycle and is defined as

$$
\Delta \triangleq \frac{t_{\text {active }}}{t_{\text {active }}+t_{\text {inactive }}}
$$

The duty cycle will be a function of the compressor capacity and the air consumption. In the simulations, the air consumption is adjusted to give a duty cycle of $20 \%$ in case the compressor is operated at a constant engine speed equal to $1400 \mathrm{rpm}$. The actual air consumption can vary quite much from one vehicle to another depending on its specification and the condition of the air system. With age, leakage in the air system, and thereby the overall air consumption, tends to increase. Further, the lifetime of the trailers is longer than for the trucks so the variation of the air system in the trailers can be even greater than for the trucks. Few measurements on the air consumption in vehicles running in actual operation were available when this work started. Therefore, in the summer 2003, two vehicle combinations in duty for a Swedish fleet where equipped with logging system that registered the pressure after the compressor. The first vehicle was a Scania R144 truck from 1998 with trailer that was going from Södertälje to Gothenburg. The second vehicle in the test was a Scania R124 tractor from 2001 with a semitrailer plus an additional second trailer, (summing up to a so called 25.25 metre combination), going from Helsingborg to Södertälje. In figure 2.9 statistics is shown on the use of the air compressor of these vehicles during the measurement period. The utilisation of the air compressor differs significantly between the vehicles although the compressors in the vehicles had the same capacity. This indicates a large difference in air consumption. It is plausible that the air system in the vehicle from 1998 was leaking to some extent.

There exist two versions of the compressor control: the traditional version with a fixed hysteresis band and the novel version with a variable 

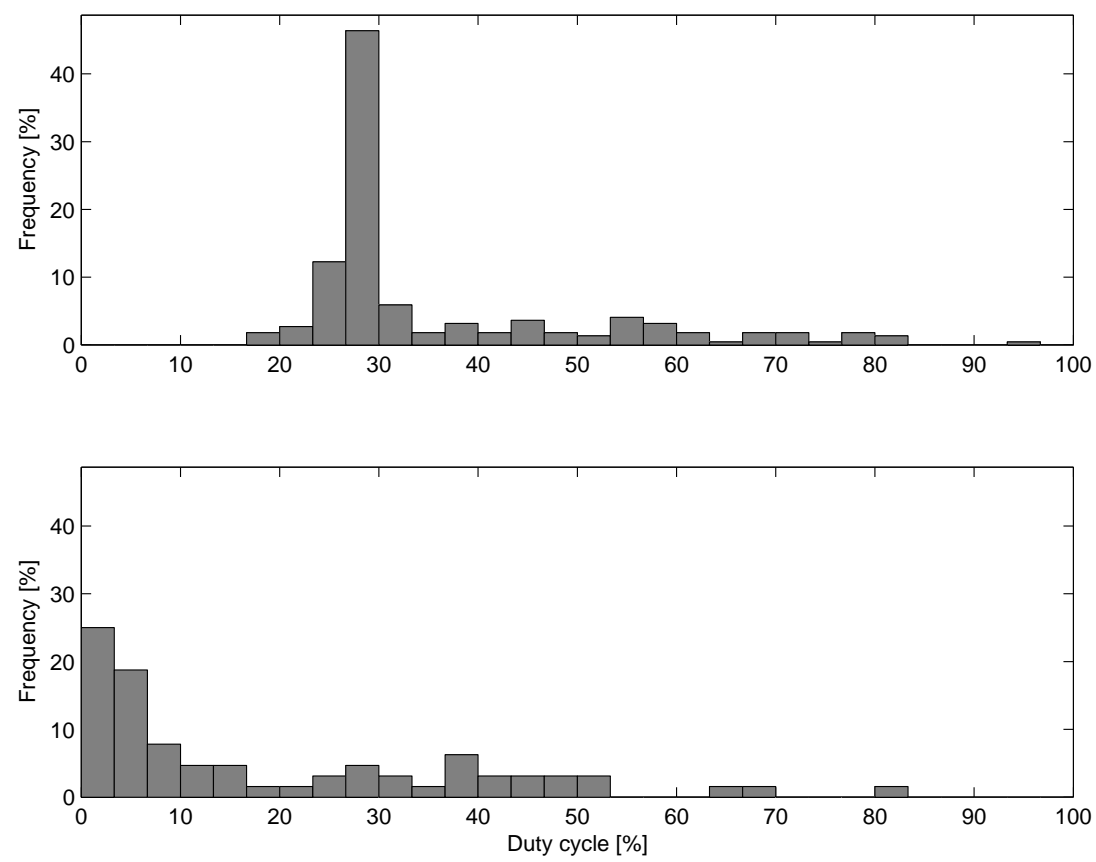

Figure 2.9: Duty cycles of the air compressor registered on a five year old truck (upper), and a two year old truck (lower).

hysteresis band. In the novel version, the hysteresis band is shifted up when the control system detects that the vehicle is running with negative torque in the driveline. Thus, when no power from the engine is required to keep the desired speed, the control system increases the reference pressure in the air tanks and additional air can be stored. When the torque in the driveline becomes positive, the hysterisis band is switched back to the original setting and the extra stored air is used up. Hence, the variable hysteresis band allows for some fuel saving since the compressor can work less often in periods when fuel is needed to drive the vehicle forward. Since the source code of the algorithm for the novel version of compressor control where not available, the described control scheme is mimicked in the simulation model to achieve an authentic behaviour. 


\section{AC compressor}

The temperature and humidity inside the cab is controlled by the climate system. The main auxiliary energy consumer in the climate system is the $\mathrm{AC}$ compressor. The $\mathrm{AC}$ compressor is used to increase the pressure of the refrigerant media. The refrigerant runs in a closed system including two heat exchangers: the condenser and the evaporator. In the condenser, the compressed gaseous refrigerant is cooled down and liquefied. Before entering the evaporator, the refrigerant passes an expansion valve where the pressure and the temperature decrease and the refrigerant is transformed back into gas. In the evaporator, heat from the ventilation air going to the cab is transferred to the refrigerant and the temperature is increased again. In the same time, the temperature and humidity of the intake air to the cab is decreased when it passes the cooled evaporator.

The interior climate in the cab is modelled as an integration of the difference between the cooling output from AC system and the heat transferred to the cab from the ambient. The magnitude of the heat transfer to the ambient is set to give a specified duty cycle of the AC compressor when the engine runs at $1400 \mathrm{rpm}$. The temperature in the cab is controlled inside a hysteresis band by switching the compressor between the active and the inactive state. In the active state the $\mathrm{AC}$ compressor is connected to the crank shaft via the belt transmission. In the inactive state an electromagnetic clutch releases the belt pulley of the compressor and the compressor goes to standstill. The power consumption of the AC compressor depends primarily on the state of the clutch and the engine speed. In the simulation, the power consumption is calculated with two look-up tables, one for the active state and one for the inactive state.

In Scania vehicles, it exists two versions of the control of the climate system: the old version is controlled via a thermostat while the new version involves an electronic control unit. The old version is tuned to always give a satisfactory dehumidification. If this would yield a too low temperature, air heated with the cooling system has to be mixed with the air blown into the cab. The new system normally works in an auto mode where primarily the temperature is controlled. This operation often yields a satisfactory dehumidification. If not, the system can be set to run in a dry mode where the operation is similar to the old version. The difference in control principle results in a rather significant difference in duty cycle. Tests performed at Scania indicates that with the new control principle, the duty cycle will go down from approximately $50 \%$ to $20 \%$ at a temperature about $25^{\circ} \mathrm{C}$ and normal humidity. Accordingly, in the simulation 
the duty cycle of the AC compressor is set to $50 \%$ for the old version and $20 \%$ for the new version.

\section{Oil pump}

The oil pump primarily provides lubrication of moving parts in the engine. Secondarily, the flow of oil is used for cooling specially exposed components inside the engine. Therefore, it is required that the pump can provide both a certain pressure and a certain flow in order to maintain a safe operation of the engine. The pump is directly connected to the engine crankshaft via a fixed gear ratio and the only active control is a pressure regulated bypass of oil internally in the pump which is active at high engine speeds. Hence, with oil which has a certain viscosity, the power consumption of the pump will be a function only of the engine speed. In the simulation model, the viscosity of the lubricant oil is assumed to be constant and correspond to the oil in the engine at working temperature. Consequently, the power consumption is calculated through a speed-dependent look-up table.

\section{Power steering pump}

The power steering provides a steering assistance which is essential especially at low vehicle speeds. The steering assistance is accomplished through a hydraulic pressure created with the power steering pump. The power consumption in the pump depends on the speed of the pump and the steering pressure. The speed of the pump is proportional to the engine speed, since the pump is belt driven. The steering pressure depends on the steering activity, the load on the steered axles and the vehicle speed.

In the model, the steering pressure is simulated as a stochastic process. The definition of the probability density function of the stochastic process originates from measurements on trucks performed at Scania during the 1990's. In the measurement the steering pressure on a $4 \times 2$ tractorsemitrailer combination was registered during driving of roads with different characters. In figure 2.10 the histogram of the steering pressure during highway driving is shown (note the logarithmic scaling of the zaxis). When driving on the highway, small steering pressures are used during a vast majority of the time. In the figure, it is also noticeable that higher steering pressures are used when the speed of the engine, and thus

likely the vehicle speed, is lower. For the simulation, a realisation of the conditional probability density function is implemented. 


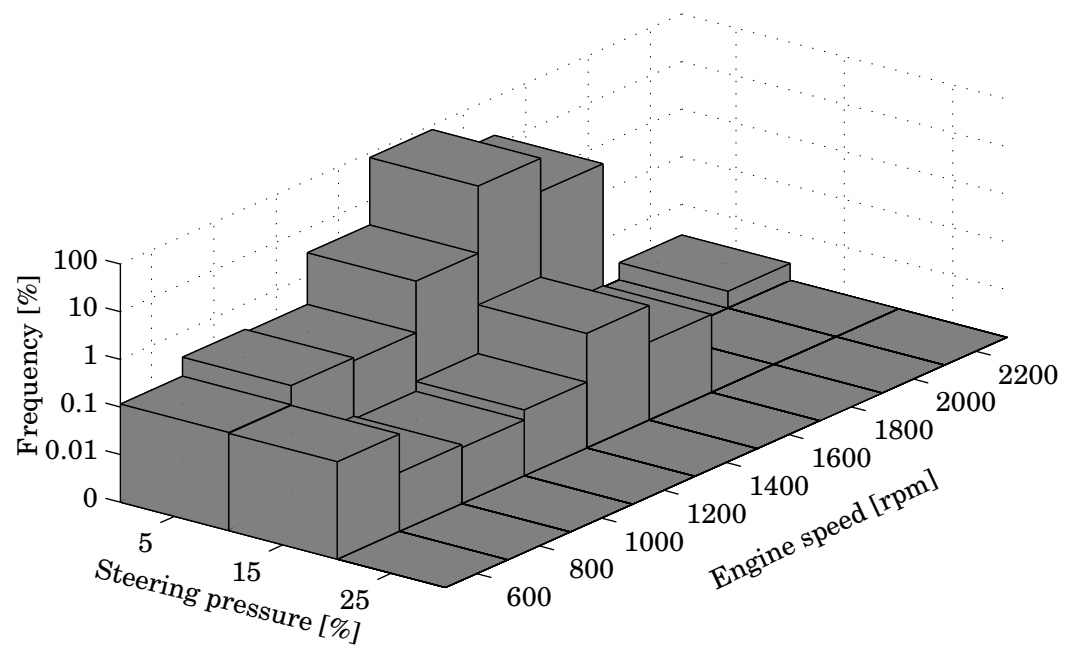

Figure 2.10: Power steering pressure registered on a $4 \times 2$ tractor with semitrailer during highway driving.

\subsection{Simulation Results}

The energy consumption of the auxiliary units is evaluated using the simulation principle and models described above. The contribution from each of the auxiliaries considered here is simulated in the two specified drive cycles. The simulation indicates that the total part of the fuel consumption that can be derived from the considered auxiliary units is in the range $4.7 \%$ to $7.3 \%$. Distributions of the relative fuel consumption of the auxiliaries, $r_{\text {aux }}$ defined in (2.3), is shown in figure 2.11 and figure 2.12 . The consumption of the individual auxiliaries ranges from $1.4 \%$ to $0.4 \%$ in the Koblenz-Trier route and from $1.7 \%$ to $0.45 \%$ in the SödertäljeGothenburg route. In the German drive cycle the auxiliaries represent a smaller part of the total fuel consumption than in the Swedish cycle. The reason for this is that the average load on the engine is higher in the German cycle, due to the greater road inclinations. Therefore the auxiliary power represents a smaller fraction of the total driveline power. 


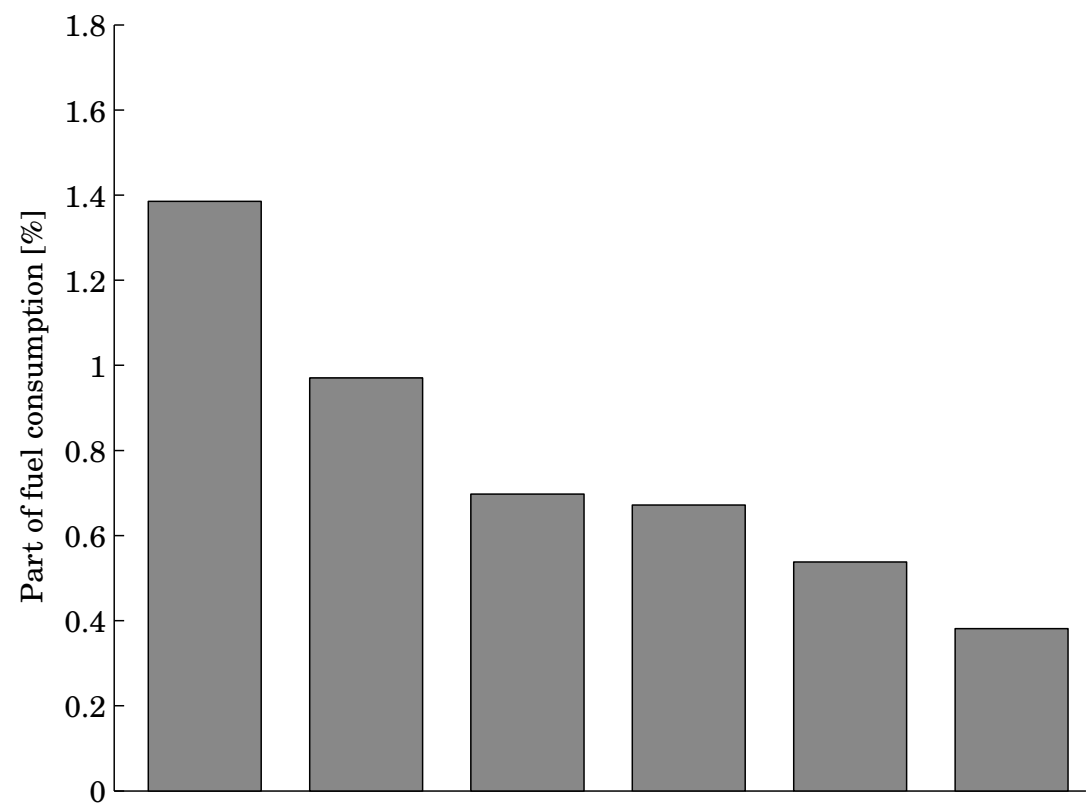

Figure 2.11: Distribution of the auxiliaries part of the fuel consumption, $r_{\text {aux }}$, simulated on the Koblenz -Trier route. The bars represent the most recent versions of the auxiliaries.

Yet, the relation between the studied auxiliaries does not change very much between the driving cases. The relation between the systems give an insight in there relative influence on the overall fuel economy.

The water pump plus the cooling fan, and the air compressor show the largest variation in absolute consumption between the drive cycles. The consumption of the cooling system is higher in the German cycle where the engine and the retarder output more power and thereby more heat than in the Swedish cycle. The consumption of the air compressor with the novel control is lower on the German cycle compared to the Swedish one. The reason for this is that the control scheme which utilise free energy when running with negative torque in the driveline is more effective in the more hilly route.

In table 2.2 , the mean power consumption, $\bar{P}_{\text {aux }}$, the mean fuel consumption, $\bar{f}_{\text {aux }}$, and the relative part of the fuel consumption $r_{\text {aux }}$ are 


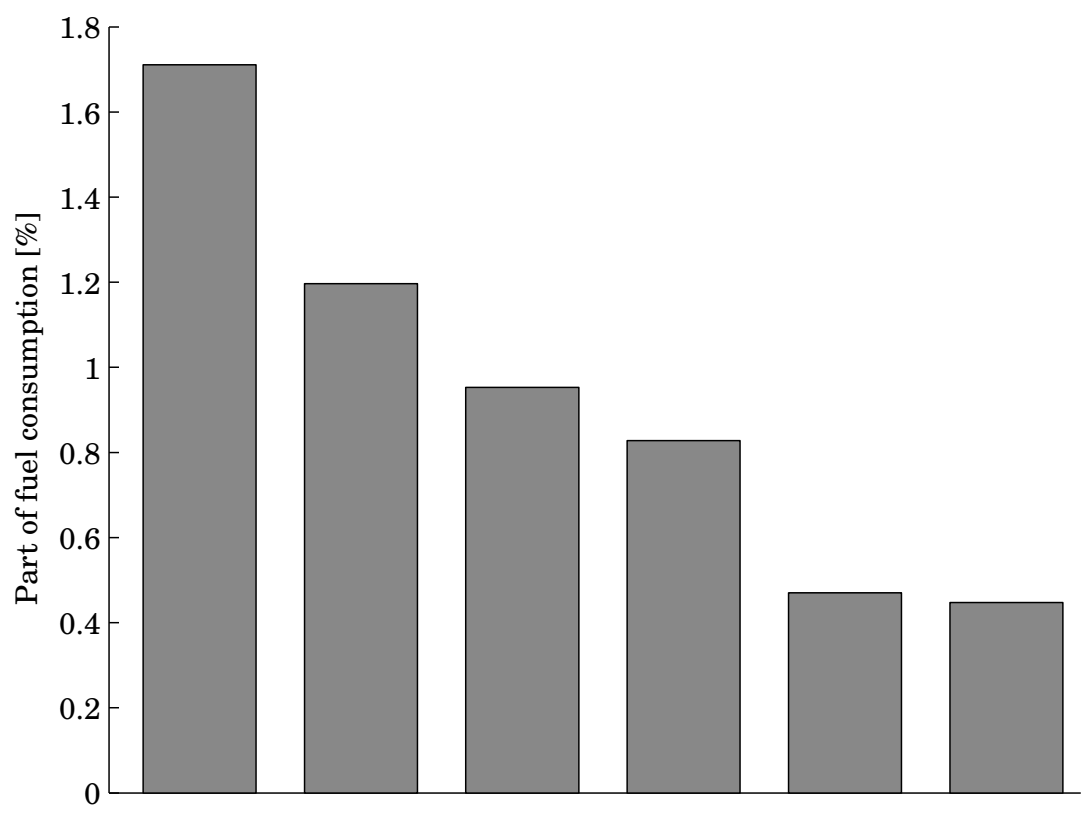

Figure 2.12: Distribution of the auxiliaries part of the fuel consumption, $r_{\text {aux }}$, simulated on the Södertälje - Gothenburg route. The bars represent the most recent versions of the auxiliaries.

summarised. The table shows the total consumptions of the auxiliaries in the studied drive cycles. The absolute consumptions of the auxiliaries are rather similar between the drive cycles. The reason for this is that the auxiliaries are delivering a certain output that is rather independent of the driving cycle.

The comparison between variants of the auxiliaries shows that some considerable improvements have been done in the newer versions. In total, the improvements represent 1.32 and 1.69 percentage point lower consumption in the German and the Swedish drive cycle, respectively. In absolute figures this corresponds to reduced fuel consumption of 0.44 and 0.53 litre $/ 100 \mathrm{~km}$, respectively. The new version of the fan clutch with a lower idle speed gives roughly a halving and two-third reduction of the consumption of the cooling system in the German and Swedish drive cycle, respectively. The improved control of the air compressor is most 
effective on the hilly German drive cycle where it yield a $22 \%$ decrease in consumption. In the Swedish cycle, the effect of the improved control is considerable lower. The refined control strategy of the AC compressor results in a $59 \%$ reduction in consumption in both drive cycles, which is approximately equal to the assumed reduction of the duty cycle.

The performed survey gives an upper limit on what fuel saving that can be achieved if the auxiliary units are redesigned. Clearly one cannot expect to gain more than the total fuel consumption of the current systems. Rather the auxiliaries are there to provide a work that is needed for the operation of the vehicle. From this study it is not possible to directly estimate what the potential improvements are if the auxiliaries were electrically driven or redesigned in other ways. In order to asses the potential, the relation between the consumption and the useful output of the auxiliaries must be further investigated. However, the following discussion illustrates some aspects regarding the potential for further improvements.

The consumption of the generator is proportional to the inverse of the efficiency. In the simulations, the efficiency of the generator is assumed to be $65 \%$. Thus, $35 \%$ of the consumption represents losses that could be reduced with an improved design. Further, if the other auxiliaries should be driven electrically, the electrical consumption would increase substantially and the efficiency of the generator would be correspondingly more critical for the consumption of the auxiliaries.

The slip speed in the fan clutch produces losses. These losses would be removed if the fan speed could be controlled directly with an electrical drive. Further, if the speed of the water pump and cooling fan could be better controlled, the blending of the coolant flow and the air flow could be adjusted to obtain a better total efficiency of the cooling system. In the case study presented in chapter 4 , an electrically driven water pump and cooling fan are used to reduce the consumption.

In the air compressor, the power losses in the inactive state of constitutes roughly $40 \%$ of the total consumption. If the compressor was electrically driven, the consumption in the inactive state would be eliminated. The corresponding effect can be achieved with a mechanical clutch installed between the engine and the compressor.

The AC compressor is deactivated with a clutch mechanism. Therefore, the power consumption in the inactive state constitutes a few percents of the average consumption of the $\mathrm{AC}$ compressor. In comparison to the air compressor, the potential for further improvements is thus lower.

The current design of the oil pump is a compromise between the 


\begin{tabular}{|c|ccc|}
\hline Drive cycle & $\begin{array}{c}\bar{P}_{\text {aux }} \\
{[\mathrm{kW}]}\end{array}$ & $\begin{array}{c}\bar{f}_{\text {aux }} \\
{[1 / 100 \mathrm{~km}]}\end{array}$ & $\begin{array}{c}r_{\text {aux }} \\
{[\%]}\end{array}$ \\
\hline Koblenz-Trier & 8.79 & 1.59 & 4.66 \\
do. with old versions & 10.67 & 2.03 & 5.98 \\
Södertälje-Gothenburg & 8.36 & 1.79 & 5.62 \\
do. with old versions & 10.51 & 2.32 & 7.31 \\
\hline
\end{tabular}

Table 2.2: Summary of the simulation result obtained on the KoblenzTrier and the Södertälje-Gothenburg routes. The total consumption obtained with the most recent versions of the auxiliaries is compared with the total consumption obtained with the older versions. The columns contain the mean power consumption $\bar{P}_{\text {aux }}$ in $\mathrm{kW}$, the mean fuel consumption $\bar{f}_{\text {aux }}$ in litre $/ 100 \mathrm{~km}$, and the relative fuel consumption $r_{\text {aux }}$ in $\%$.

pressure required for lubrication and the flow required for cooling. The control freedom obtained with an electrical drive of the pump, or a hybrid between a mechanical and electrical drive, could be used to separate these requirements. If the oil pressure and the oil flow could be controlled tighter to the required limits, the consumption would be reduced.

The power steering deliver very little useful output during most parts of the high-speed driving but the consumption is not negligible. At higher speeds corresponding to highway driving, most of the hydraulic oil is bypassed internally in the pump. This results in energy losses. In a pure electrical or a electro-hydraulic power steering these losses, would be removed. 



\section{Chapter 3}

\section{Modelling of Energy Consumption}

In this chapter vehicle models that can be used to evaluate energy consumption of the auxiliary units are presented. With aid of the simulation models, the potential energy savings of new designs can be assessed. Here the ideas behind development and maintenance of a comprehensive model library are presented. The library is developed in Modelica, ([Mod02]). Modelica is an object oriented modelling language well suited to describe behaviour of complex systems containing parts from different engineering disciplines, e.g., mechanics and electronics. Figure 3.1 shows the composition of the model at the highest level. The development of the Modelica library is a continuous activity at Scania which branch off into some different directions. In the models of the library, the vehicle is set to drive a road with varying topology and speed limit that have been obtained from recordings of real roads. The vehicle is assumed to run on cruise control and with computer-controlled gear shifting (automated manual transmission). Algorithms from the production version of the control are incorporated in the simulation model. The principle for how the drive cycles are defined and how the control of the drive line is done is further described in sections 2.2 and 2.4. Prior to this work, the vehicle model has been validated with respect to the energy consumption of the combustion engine and losses such as rolling resistance and air drag, [San01]. Influences from the cooling system, and other auxiliary units, were only included as a lumped effect on the net fuel consumption. The work pre- 


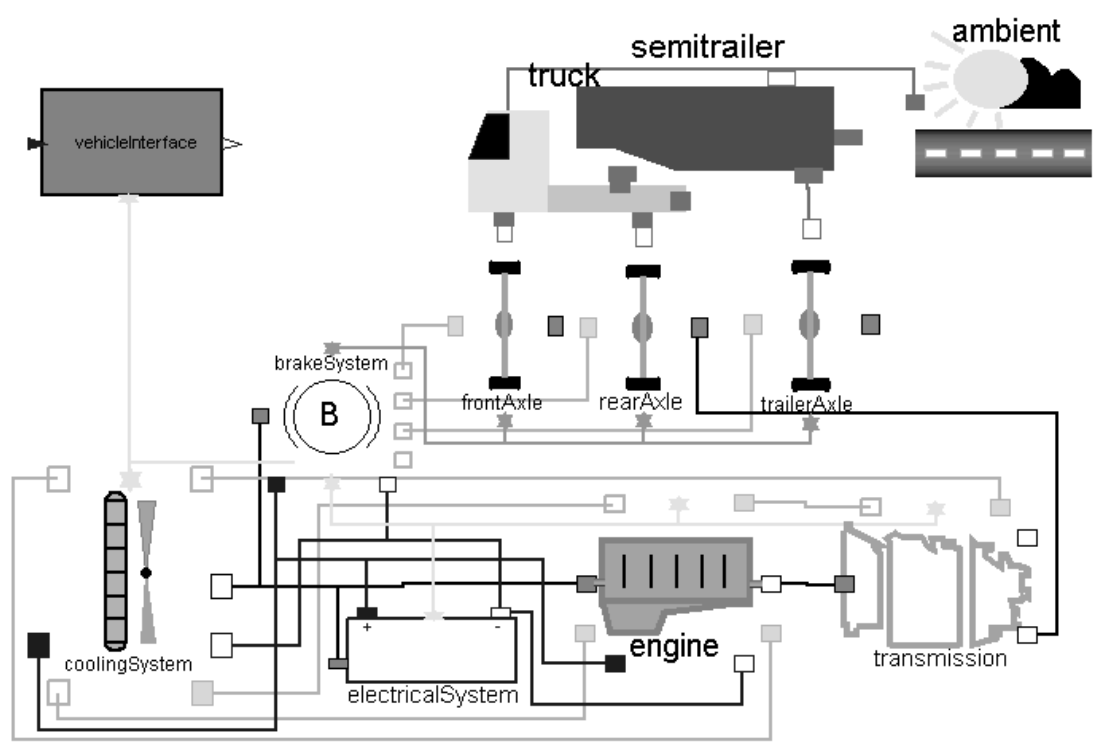

Figure 3.1: Modules of the simulation model.

sented in this chapter refines the description of the cooling system.

In section 3.1, the purpose of this type of simulation models is discussed. The organisation of the model library is described in section 3.2. Section 3.3 describes the modelling of the cooling system in some detail. Sub-models are built from physical principles, resulting in grey-box models with parameters identified from various tests in a laboratory environment. Assembling of the sub-models into a complete model is described in section 3.4. Measurements from tests in a wind tunnel are used to tune the performance of the total model. The model is validated against data recorded from a dynamic drive cycle in the wind tunnel.

\subsection{Selection of Model Structure}

The prime goal with the vehicle model is to serve as a tool for study effects on the fuel economy from alternative designs of sub-systems. The main quantity that is studied is flow of energy between various parts 


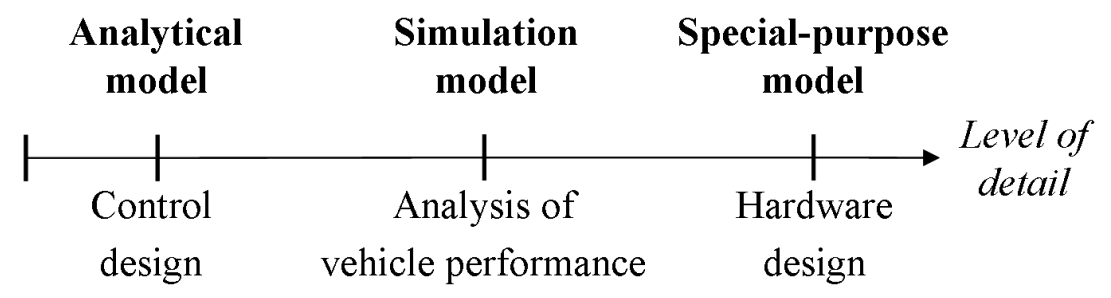

Figure 3.2: Classes of models used in the automotive industry.

of the vehicle. To give an accurate estimate of the energy balance, the model must cover the whole vehicle and describe processes involved in the energy conversion with a significant level of detail. Besides description of physical phenomena, it contains control software and various look-up tables. We refer to this type of model as simulation models since it (in contrast to simpler models of sub-systems or black-box models of complete processes) might not be possible to summarise the model in a set of mathematical equations. In the literature this type of models sometimes are referred to as software models (cf., [Lju99]). Although the model might be comprehensive, by necessity it must in many parts be a rough approximation in order to keep the complexity on a reasonable level; fast dynamics and other effects not relevant for the study may be neglected. Models used in vehicle development can be classified in three groups according to which level of detail they use to describe the physical world, see figure 3.2. The most detailed models, referred to as special-purpose models, are used for the design of hardware components. In this category one find models of the combustion process, 3 -D models of the suspension etc. On the other extreme we find models used for control design. Since their limited extent allows for an analytical description in closed form we denote these models analytical models. Often analytical models represent an abstract image of the physical world. Parts may be lumped into a single quantity, e.g., total inertia of bodies which are mechanically connected to each other, blurring out the interfaces between components in the system described in the model. Similarly to special-purpose models, analytical models typically cover a limited part of the vehicle. 


\section{Simulation models}

It is more and more important to perform simulations covering the complete behaviour of the vehicle as control functions become more integrated. Beside this, overall performance parameters as fuel consumption, electric energy balance and cooling capacity have become more critical in vehicle design. Models including relevant descriptions of major parts of the vehicle tend to grow in complexity. To assemble the total model in a closed form of equation might not be possible. Moreover, often not only the input-output behaviour of the model is of interest. Internal signals may be just as important to study. Therefore it is preferable to split up the model in components corresponding to physical objects and derive sub-models from first principles. Structured in this way, simulation models are intrinsically multi-domain. Although all the classes of models play a specific part, it is advantageous to organise the modelling effort in an integrated way. Clearly, there exists a possibility to utilise results gained working with one type of model also for other type of models. From the perspective of simulation models, an outline of interactions with analytical and special-purpose models might include:

1. When assembling simulation models, time will be saved if modelling paradigms and measurement data can be adopted from the teams working with special purpose models.

2. Analytical and simulation models can share databases for parameter values. In case either of them work with a lumped definition of parameters, it may be possible to implement translation rules for transferring one type of parameter set to the other.

3. Control algorithms developed based on an analytical model can be tested together with a simulation model containing more details before the control is applied in a real vehicle.

4. To gain insight in dependencies between parts of the model or to do sensitivity analysis of parameter influences when working with complex simulation models, one often have to resort to a simplified analytical model.

5. The work with simulation models can give useful input to the hardware design. Results from a complete vehicle simulation can be used to define operating profiles for the components such as average speed, time spent on a certain load, etc. 


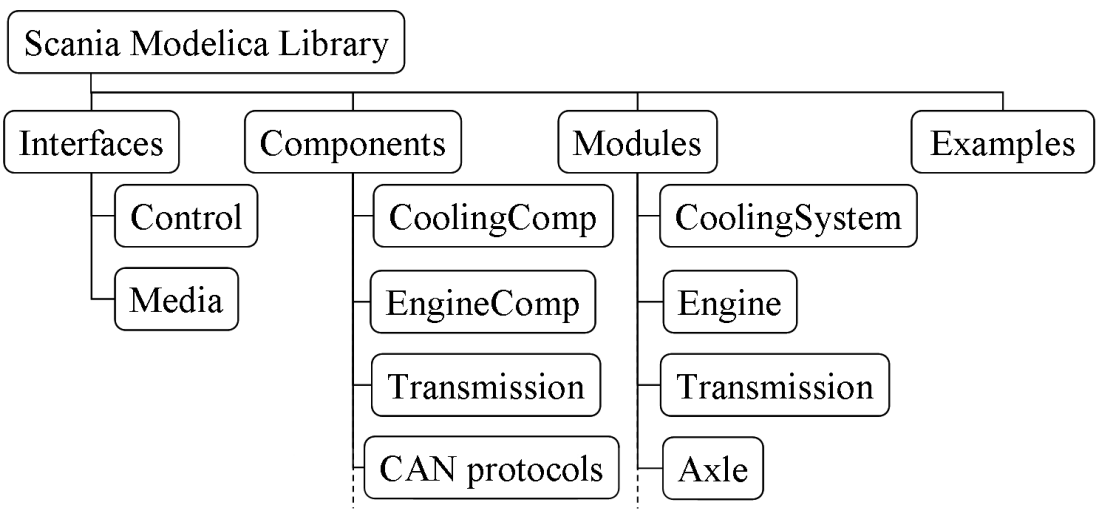

Figure 3.3: Structure of the Scania Modelica Library.

\subsection{Model Library}

A library of simulation models is developed according to the principles described in the previous section. In contrast to the Modelica Standard Library, the library is not organised in different engineering disciplines. Instead it is organised after the parts of the truck. The library consists of four main branches: Interfaces, Components, Modules and Examples. The principal structure of the library can be viewed in figure 3.3. The Interface branch contains classes describing connections between model components. Although the library relies heavily on connector classes defined in the Modelica Standard Library, some unique connectors are defined. One example is the CAN connector, used to mimic the information flow between control units in the truck. Further, under the Interfaces sub-library Media, base classes for thermodynamic and hydraulic models are found. These base classes are mainly used in models of components in the cooling system. In the thermodynamic and hydraulic base classes many of the modelling ideas used are adopted from the Modelica library ThermoFluid developed by Tummescheit et al. [TEW00]. However, here a somewhat simpler structure and less extensive description of media properties are used. In the Components branch models of all physical parts needed to build up the complete model of a truck are gathered. Modules, in the next branch, are a higher level of abstraction, and contain more compound models. The idea is to define a set of generic modules 


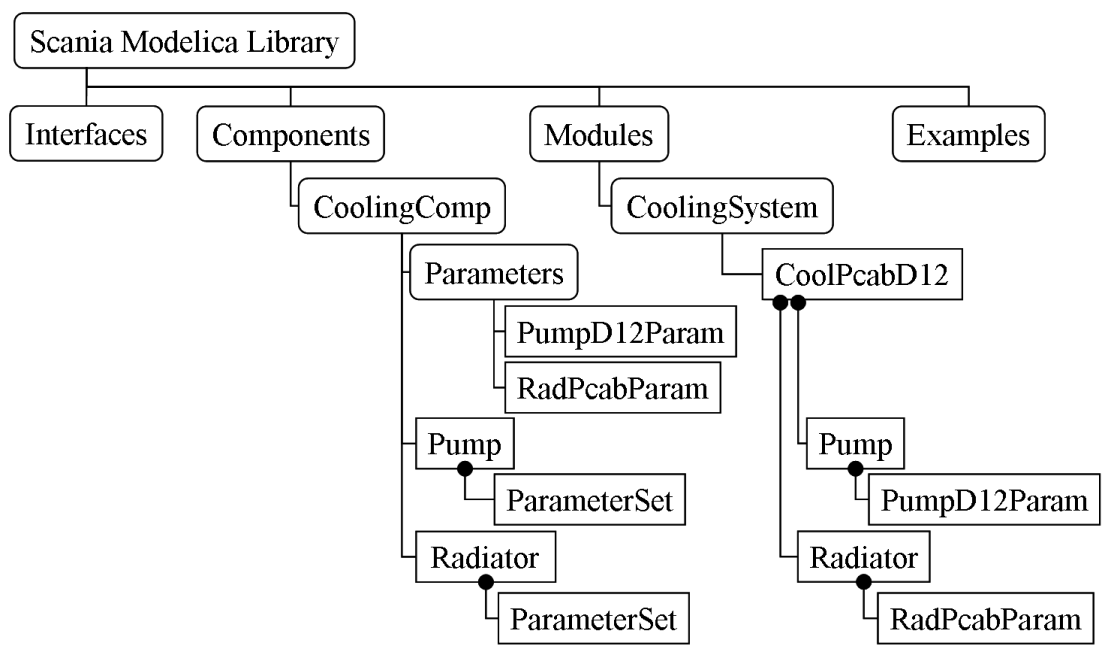

Figure 3.4: Parameterisation of the model exemplified with the cooling module.

with well-defined interfaces that can be used for simulations with various purposes. In the last branch a number of working examples is built that can be used directly for simulations. Figure 3.4 illustrates how the models are parameterised to obtain modules that correspond to physical modules. In the figure, the parameterisation of the cooling module is exemplified. Each component contains a placeholder for a set of parameters of a defined structure. Parameter sets with values describing various versions of the components are gathered in sub-libraries. When modules are put together, the generic placeholders are replaced with the parameter set of the current versions of components.

\subsection{Cooling System Module}

The cooling system is one of the modules of the vehicle library. Energy consumers in the cooling system are primarily the cooling fan and the water pump. In heavy vehicles, these units normally are mechanically driven. The model corresponds to the current design of a Scania truck where the water pump is directly driven from the crankshaft while the cooling fan is connected to the shaft via a viscous clutch enabling a passive 


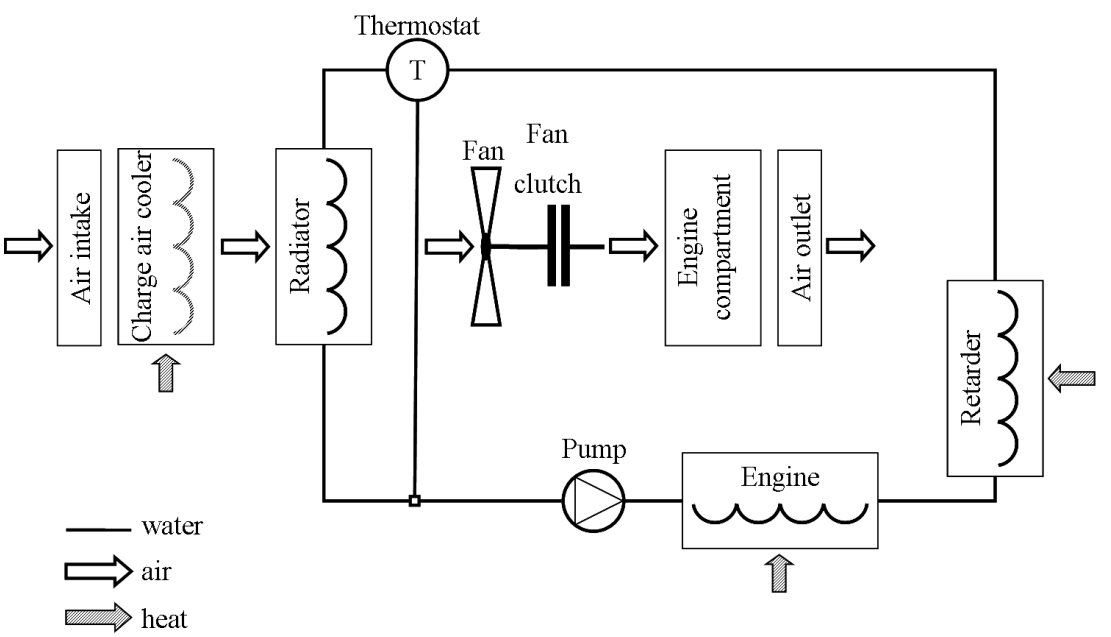

Figure 3.5: Components in the cooling system module.

speed control. However, the basic structure allows for changing the model to describe other ways of driving and controlling these auxiliaries.

\section{Cooling system components}

In figure 3.5 the structure of the cooling system is depicted. The model mainly consists of two adjoining flows of mass and energy: the flow of coolant fluid and the airflow. A more detailed description of the cooling system is given in section 2.5. The main parts of the cooling system are modelled using the thermodynamic and hydraulic base classes in the Media sub-library of the library. The modelling is rather standard and essentially follows the principles in ThermoFluid [TEW00]. The thermodynamic models are built up with alternating control volumes and flow models. Principally, mass and energy balances are defined in the control volumes, while relations between the pressure drop and the flow are determined in the flow models. Series of alternating control volumes and flow models can be aggregated to form a composite model. In figure 3.6 the principle of alternating control volumes and flow models is illustrated. 


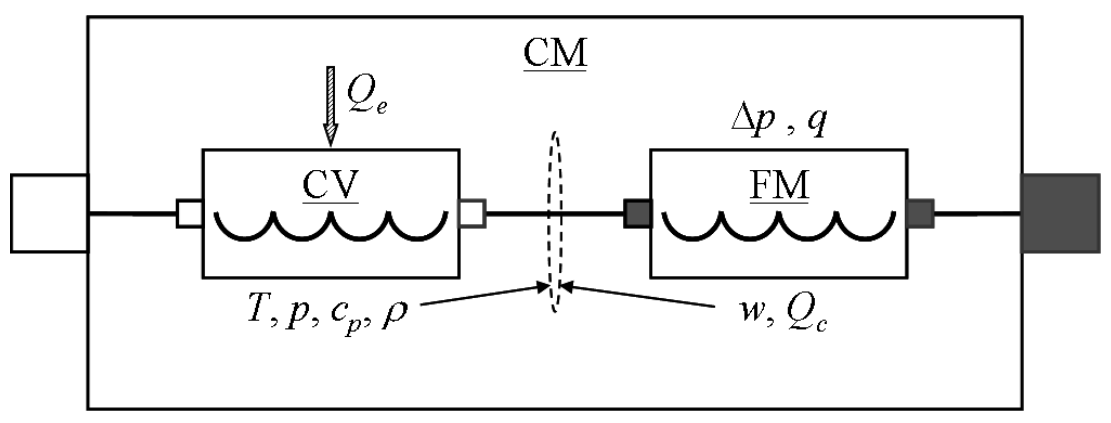

Figure 3.6: The thermo-hydraulic models of the cooling system are built up with alternating control volumes (CV) and flow models (FM). Series of control volumes and flow models are aggregated to composite models $(\mathrm{CM})$. The effort variables in the interface between the models, e.g. $T$, are determined in the control volume and flow variables are determined in the flow model.

The Modelica definition of the connector class defining the interface between the models is:

\section{connector BaseHose}

Slunits.Temp_K T "temperature";

Slunits.AbsolutePressure $\mathrm{p}$ "absolute pressure";

Slunits.SpecificHeatCapacity cp "heat capacity";

Slunits.Density rho "density";

flow Slunits. MassFlowRate mdot "mass flow";

flow Slunits.Power Qconv "convective heat flow";

end BaseHose;

In the subsequent description, the variables defined in the interface will be denoted with $T, p, c_{p}, \rho, w$ and $Q_{c}$, respectively. The effort variables in the interface, e.g. $T$ and $p$, are determined from equations in the control volumes while the flow variables, $w$ and $Q_{c}$, are determined in the flow models. To distinguish between the types of models, non-filled icons are used for connectors to control volumes while filled icons are used for connectors to flow models. Consequently, composite models have one nonfilled icon at the side neighbouring to the first control volume in the model and one filled icon neighbouring to the last flow model inside the model. 


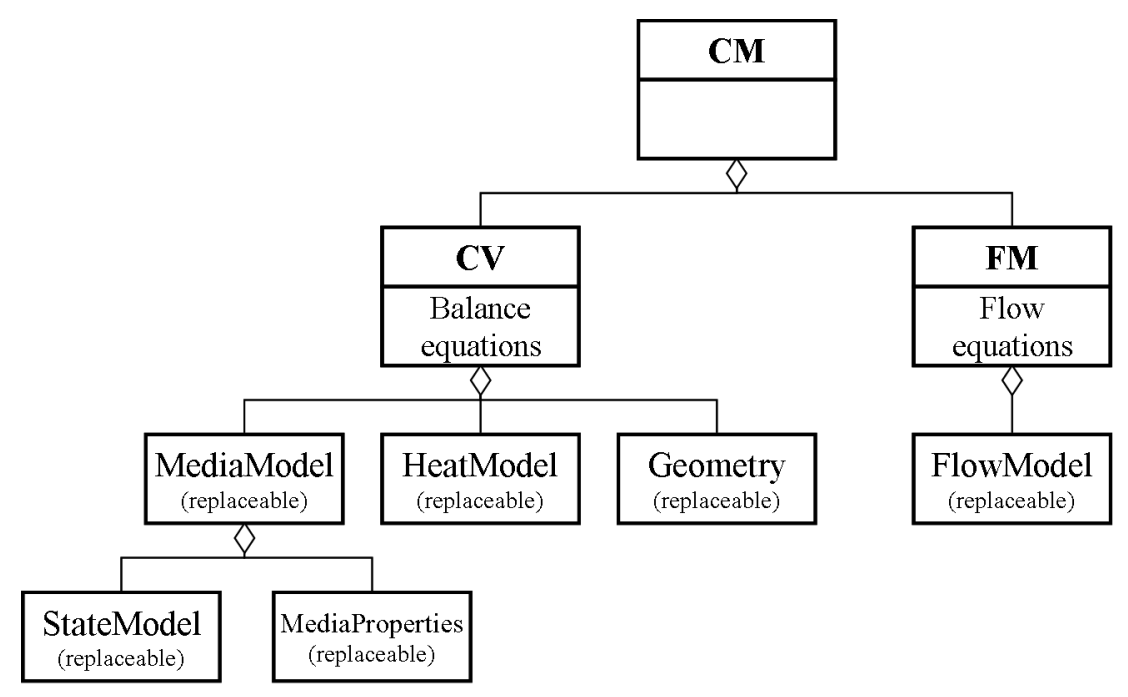

Figure 3.7: Class diagram of the base classes used in the cooling system. Composite models (CM) are an aggregate of control volumes (CV) and flow models (FM). Selectable properties of the control volume and the flow model are defined in replaceable sub-models.

A class diagram showing the decomposition of the thermodynamic base classes is shown in figure 3.7. The compound model is aggregated from control volumes and flow models. The control volume contains a standard set of balance equations present in all thermodynamic models which inherits from the base class. Relations specific for the actual type of component is placed in replaceable sub-models. Analogously, the base flow model contains the common flow equation while specific flow characteristic resides in a replaceable sub-model.

\section{Control volumes}

The balance equations included in all control volumes, as illustrated in figure 3.7, define the mass and energy balance. Let $\dot{m}$ and $\dot{U}$ denote the time derivative of mass and internal energy, respectively, and $V$ the volume of the control volume. For a volume with one upstream and one downstream 
connection to the surrounding components, the balance equations are

$$
\begin{aligned}
& \dot{m}=w^{u}-w^{d} \\
& \dot{U}=Q_{c}^{u}-Q_{c}^{d}+Q_{e}-p \dot{V}
\end{aligned}
$$

Here $w^{u}$ and $w^{d}$ denote the mass flow in the upstream and downstream connection, respectively. Analogously, $Q_{c}^{u}$ and $Q_{c}^{d}$ denote the convective heat flow in the connections. Heat transferred to the volume through for example conductance is denoted with $Q_{e}$.

The replaceable sub-model MediaModel showed in figure 3.7 make it possible to select the type of media and the state representation that should be used in the control volume. The state transformation from the primary mass and energy balances is defined in the replaceable model StateModel while the properties of the media contained in the control volume is defined in MediaProperties. Thus, combining instances of StateModel with instances of MediaProperties, a wide variety of medias with various state representation can be realised. In the sub-model, HeatModel, the heat exchange with the surrounding is defined, and the model Geometry defines the geometry of the volume. Models of heat exchange with surrounding component is described in the sequel section on heat conductance. The geometry in most of the components set to constant.

For the airflow, pressure, $p$, and temperature, $T$, are chosen as state variables. The dynamics which are implemented in the StateModel of the airflow then become

$$
\begin{aligned}
\dot{m} & =V \frac{\partial \rho}{\partial p} \dot{p}+V \frac{\partial \rho}{\partial T} \dot{T}+\rho \dot{V} \\
\dot{U} & =m \frac{\partial u}{\partial p} \dot{p}+m \frac{\partial u}{\partial T} \dot{T}
\end{aligned}
$$

The boundary conditions of the airflow in the front and the rear of the truck cab are described as a special variant of StateModel which do not contain dynamics. Instead the pressure and temperature is defined in relation to the ambient. The wind speed gives rise to a differential pressure at the air intake and outlet relative to the ambient pressure. The temperature is assumed to be equal over the boundary. In the model, the pressure difference depends on the wind speed, $v$, the air density, $\rho$, and the non-dimensional coefficient of air drag $C D$. 
The expressions for the pressure and the temperature at the air intake and outlet are

$$
\begin{aligned}
p & =p_{a} \pm C D \frac{\rho}{2} v^{2} \\
T & =T_{a}
\end{aligned}
$$

where $p_{a}$ and $T_{a}$ are the pressure and temperature of the ambient, respectively. The plus sign in the equation in (3.3) corresponds to the air intake while the minus sign corresponds to the air outlet. Thus, the differential pressure in the front and the rear of the cab cause the airflow to increase through the cooling system. The sub-model MediaProperties of the control volume contains expressions of the partial derivatives and the density $\rho$ in the state equations. Since the air is regarded as an ideal gas, the expressions in (3.2) become

$$
\begin{array}{lll}
\frac{\partial u}{\partial p}=0, & \frac{\partial \rho}{\partial p}=\frac{M}{T R}, & \rho=\frac{p M}{T R} \\
\frac{\partial u}{\partial T}=c_{v}, & \frac{\partial \rho}{\partial T}=\frac{p M}{T^{2} R}
\end{array}
$$

Here $M$ denotes the molar mass and $c_{v}$ the specific heat capacity at constant volume, respectively, while $R$ is the molar gas constant. The state equations and the media properties used for the coolant are similar to equation (3.2) and (3.4), although only the temperature is chosen as state variable. The pressure of the coolant is determined purely from static hydraulic relationships defined in the flow models.

\section{Flow models}

In the flow models, primarily relations between the pressure and the volume flow are described. The relations depend on the actual type of the model and are for that reason implemented in a replaceable submodel of the flow model. Equations that determine the volume flow rate and the convective heat flow are given in a base class which is common for all the flow models. For a flow model with one upstream and one downstream connection to the surrounding components, the volume flow rate is expressed as

$$
q^{d}= \begin{cases}\frac{w^{u}}{\rho^{u}} & \text { if } w^{u}>0 \\ \frac{w^{d}}{\rho^{d}} & \text { otherwise }\end{cases}
$$


while the convective heat flow is given by

$$
Q_{c}^{d}= \begin{cases}w^{u} c_{p}^{u} T^{u} & \text { if } w^{u}>0 \\ w^{d} c_{p}^{d} T^{d} & \text { otherwise }\end{cases}
$$

Here the super-scripts $u$ and $d$ refer to variables associated with the upstream and downstream connector, respectively.

The pressure-flow relation determines the pressure difference $\Delta p=$ $p^{u}-p^{d}$ between the connections of the component. Mainly two types of pressure-flow relations are implemented in the flow models: pressure losses and pressure increase in pumps and fans. For the airflow, pressure losses in the components along the flow path are modelled as an exponential friction loss

$$
\Delta p=c|q| w^{e}
$$

The frictional pressure losses in the coolant path is modelled with second order polynomials

$$
\Delta p=c_{2}|q| q+c_{1} q
$$

The pressure rise in the pump and the fan depend on the flow through the components and the angular velocity of the shaft. In the model the following equation describes the operation of the pump:

$$
\Delta p=R_{1} \rho|\omega| \omega+2 R_{2} \omega w-R_{3}|q| w
$$

The operation of the pump is modelled with

$$
\Delta p=R_{1}|\omega| \omega+2 R_{2} \omega q-R_{3}|q| q
$$

In equations (3.9) and (3.10), $\omega$ denotes the angular velocity in the mechanical interface of the pump or the fan. The torque in the interface is derived through the relation

$$
\Delta p q=\eta T_{s} \omega
$$

where $T_{s}$ denotes the torque on the shaft. For both the pump and the fan, the efficiency $\eta$ is modelled as

$$
\eta=\eta_{0}\left(c_{\eta_{2}} \frac{q^{2}}{\omega^{2}}+c_{\eta_{1}} \frac{|q|}{|\omega|}+c_{\eta_{0}}\right)
$$




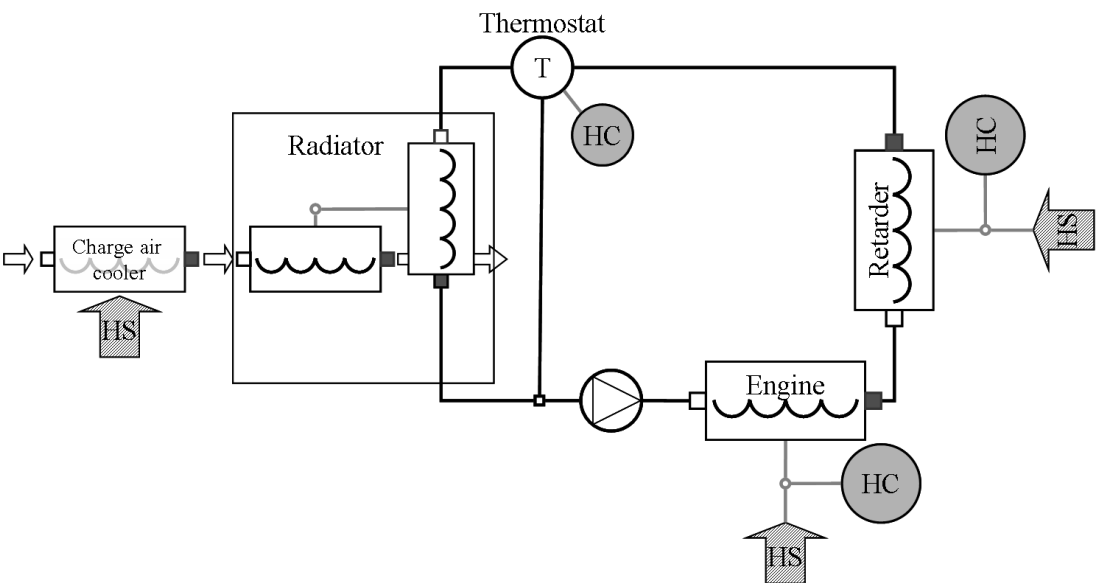

Figure 3.8: Illustration of heat conductance between parts of the model. Heat sources (HS) is connected to heat capacitances (HC) and the thermo-dynamic models in the engine and the retarder. In the thermostat, heat is transferred between the coolant flow and a heat capacitance representing the dynamics of the thermostat. In the radiator, heat is transferred between the coolant flow and the airflow.

\section{Heat conductance models}

In figure 3.8, the heat conductance included in the model is illustrated. Heat conductance is taking place between the airflow and the coolant flow in the radiator, and between the coolant flow and external heat capacitances in the engine, the retarder and the thermostat. The heat capacitances represent storing of heat in bodies not included in the thermodynamic models and are contributing to the overall dynamics of the cooling system. The dynamic state of the heat capacitances is the temperature, which is obtained through the following integration of the net heat flow

$$
c_{p b} m_{b} \dot{T}_{b}=\sum_{i} Q_{i}
$$

Here $c_{p b}$ and $m_{b}$ represent the specific heat capacitance and the mass of the body, respectively, and $Q_{i}$ is the heat transfer in the $i$ :th connection to the body. The heat capacitances in the engine and the retarder model are connected to the coolant flow and to heat sources representing the 
heat emitted from the combustion and the retarder braking, respectively. In the thermostat, heat is transferred between the coolant flow and a heat capacitance representing the wax body inside the thermostat. The heat sources shown in figure 3.8 are heat losses that need to be cooled away. The engine emits heat to the cooling system both directly into the engine block, which is heated up by the combustion, and through the cooling of the charge air. The amount of heat depends on the current torque and speed of the engine. In the model the heat flows are calculated from look-up tables. The tables are obtained from measurements done in test cells. The heat emitted to the cooling system from the retarder is directly proportional to the braking power.

The heat conductance in the engine, the retarder and the thermostat is assumed to depend linearly on the difference in temperature of the heat capacitances and the coolant in the corresponding components. Thus, the amount of external heat, $Q_{e}$, entering the balance equation (3.1) of the thermo-dynamic model is expressed as

$$
Q_{e}=\lambda\left(T_{b}-T\right)
$$

where $T_{b}$ and $T$ is the temperature of the heat capacitance and the control volume, respectively.

The model of the heat conductance in the radiator is more complex. It is based on the specific heat transfer per unit of temperature difference between the coolant flow and the air flow entering the radiator, respectively. The specific heat transfer, denoted $Q_{s}$, is assumed to be a function of the air mass flow and the coolant mass flow. Let the entry temperatures of the coolant flow and the air flow be denoted with $T_{c}$ and $T_{a}$, respectively. Then the net heat transfer in the radiator, $Q_{e}$, can be expressed as

$$
Q_{e}=\left(T_{c}-T_{a}\right) Q_{s}\left(w_{c}, w_{a}\right)
$$

where the $w_{c}$ and $w_{a}$ denotes the mass flow of coolant and air, respectively.

The specific heat transfer $Q_{s}\left(w_{c}, w_{a}\right)$ is calculated through interpolation of tabulated data. The specific heat transfer is measured as a standard procedure when the radiator is designed. However, the measurement data typically do not covers the complete range of mass flows that can be expected in the simulation. During design of the radiator, the main focus is on the maximum capacity and consequently data is normally available only for high mass flows while data for low mass flows are lacking. In order to compensate for this, a physical model of the heat exchanger is 


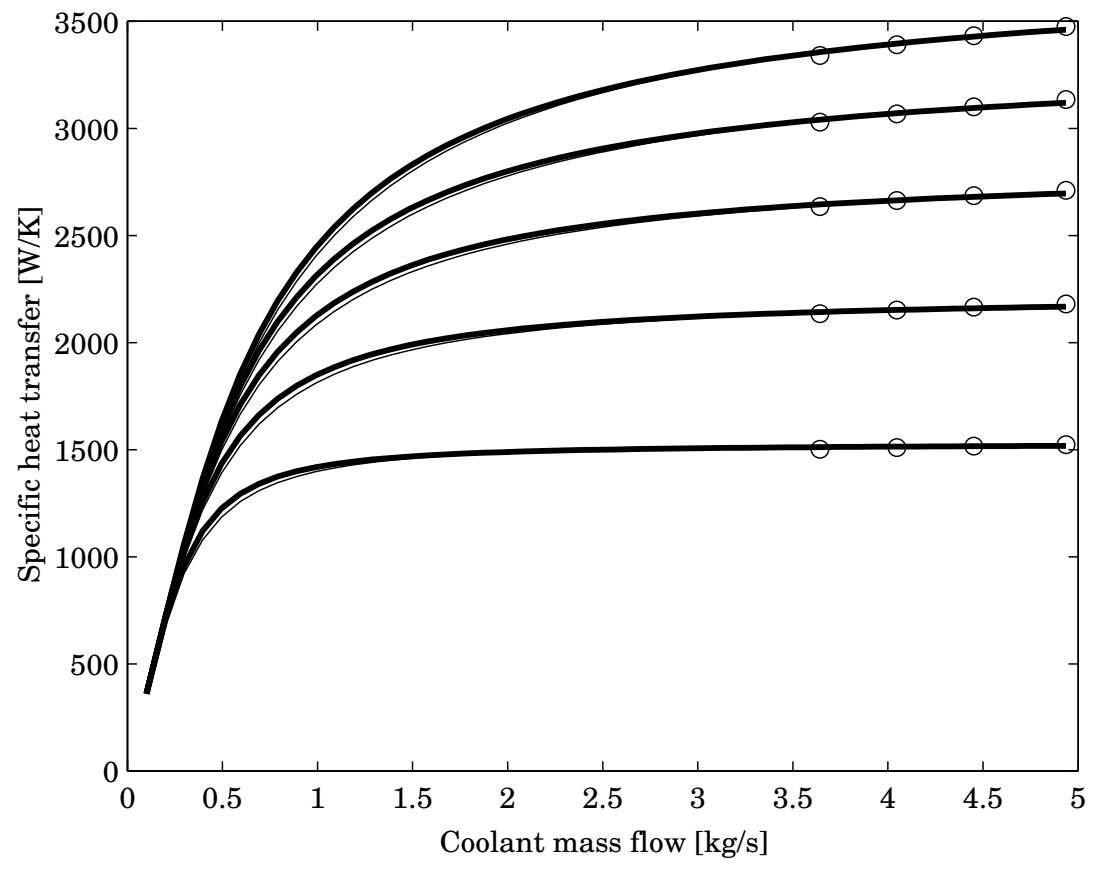

Figure 3.9: Measured specific heat transfer in the radiator (circles) compared with heat transfer predicted by the non-dimensional model (thick solid) and the PDE-model (thin solid).

used to generate values of $Q_{s}$ for the range where no data are available. This means that the model will be used outside the range where the parameters were estimated. This is generally doubtful since very little can be said about the agreement between the model output and the behaviour of the real system. In order to compensate for this deficiency, the outputs of two different physical models have been compared. The first physical model is based on a straight forward Euler discretisation of the spatial partial-differential equation (PDE) of the heat transfer in steady state. The PDE is obtained by considering the energy balance over the surface of the heat exchanger. The second model is a non-dimensional expression for cross directional heat exchangers with parallel paths found in for instance [VDI94]. The two physical models at hand are judged to 
be too complex to be used directly in the simulations. Instead the models are employed to generate tables which cover the complete range of expected mass flows. The main parameter to estimate in both models is the specific heat conductance per unit surface area, denoted $\lambda$. In order to take into account that the airflow is unequally distributed over the surface, a correction factor for the air flow is introduced. Employing the correction factor, $r_{a}$, the airflow used as input to the models is $w_{a}^{\prime}=r_{a} w_{a}$. Consequently, the parameters that are estimated from the available measurement data are $\lambda$ and $r_{a}$.

Figure 3.9 shows the measured specific heat transfer and the predicted output of the models. A good agreement is obtained in the region where measurement data is available and both physical models give similar predictions for mass flows where measurements are lacking. The non-dimensional model from [VDI94] is the one that is used to generate the data which is put into the simulations.

Equivalent expressions for the heat transfer in heat exchangers are used in for instance the simulation software Kuli [Ste04] which is a specialised tool for simulation of automotive cooling systems. An alternative modelling paradigm for the heat exchanger is the use of the log-mean temperature, see e.g. [PG84]. For cross directional heat exchangers the analytic derivation of the log-mean does not directly apply but the concept could be extended to this type of heat exchangers by introducing correction factors which typically is estimated from measurement data. However, in transient simulations, the expressions for the log-mean temperature might become singular. This is recognised in for instance the work by Tummescheit [Tum02] where some numerical methods to deal with this is proposed. However, since this is less straightforward and is not expected to yield a better accuracy, the use of expression (3.15) is judged to be a preferable solution.

\section{Parameter estimation}

Some of the parameters in the sub-models represent basic quantities such as mass or volume. No explicit estimation of these parameters is needed, since they could be found in the technical specification of the components. Other parameters, typically describing the behaviour of the flow models, have to be estimated from data. For that reason, experimental data is collected from tests on individual components in a laboratory environment. For each component, a small identification problem is solved trying to fit the predicted output from the models to the measurements. 


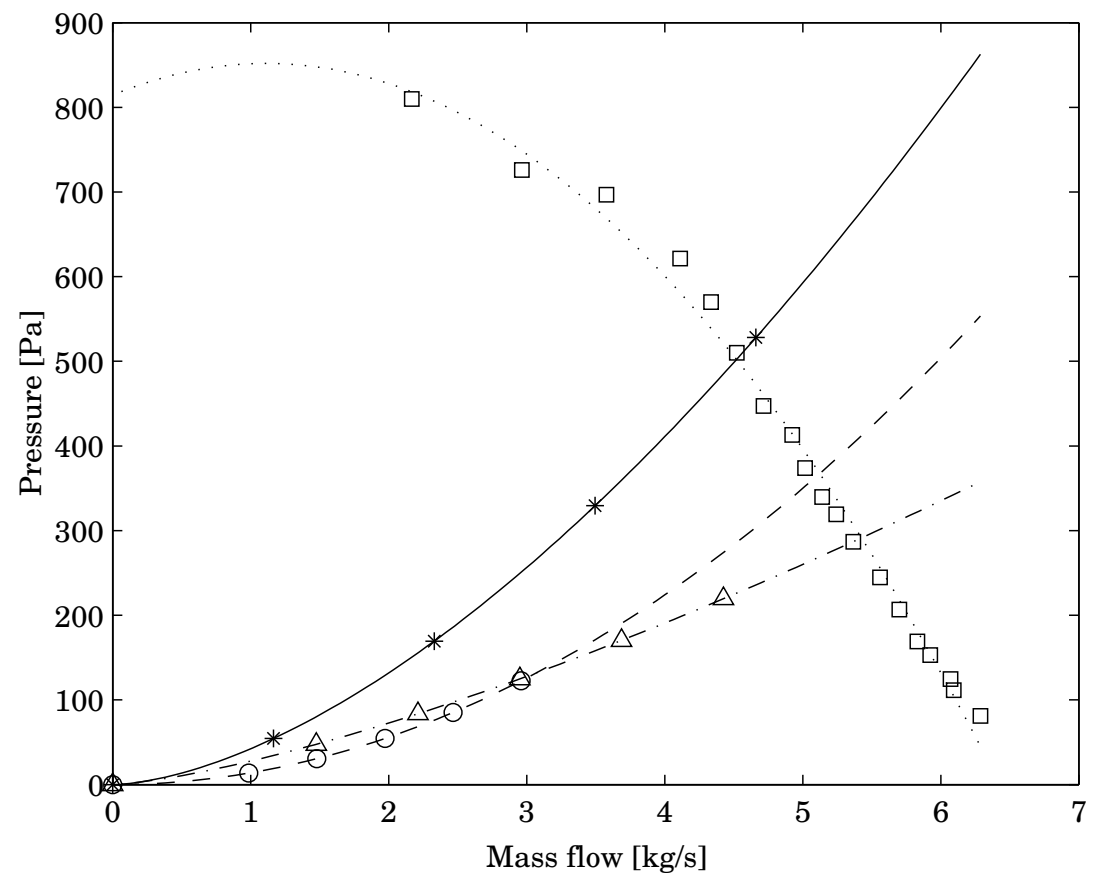

Figure 3.10: Pressure drop as a function of airflow for the charge air cooler model (solid) compared with measurements (stars). Corresponding drops for radiator (dashed and triangles), and engine compartment (dashdotted and circles). Pressure rise of the fan model (dotted) at $1400 \mathrm{rpm}$ compared with measurements (squares).

Essentially parameters of equation (3.7)-(3.3) and other characteristics are identified for each component depicted in the overview of the cooling module in figure 3.5. Table 3.1 summarises which parameters that are identified and what data that are used. The tests are performed in the laboratory under well-controlled conditions. As a result the obtained prediction errors are very small as can be seen in figure 3.10, showing the pressure drops in the airflow path and in fugure 3.11 showing the pressure in the coolant path. 


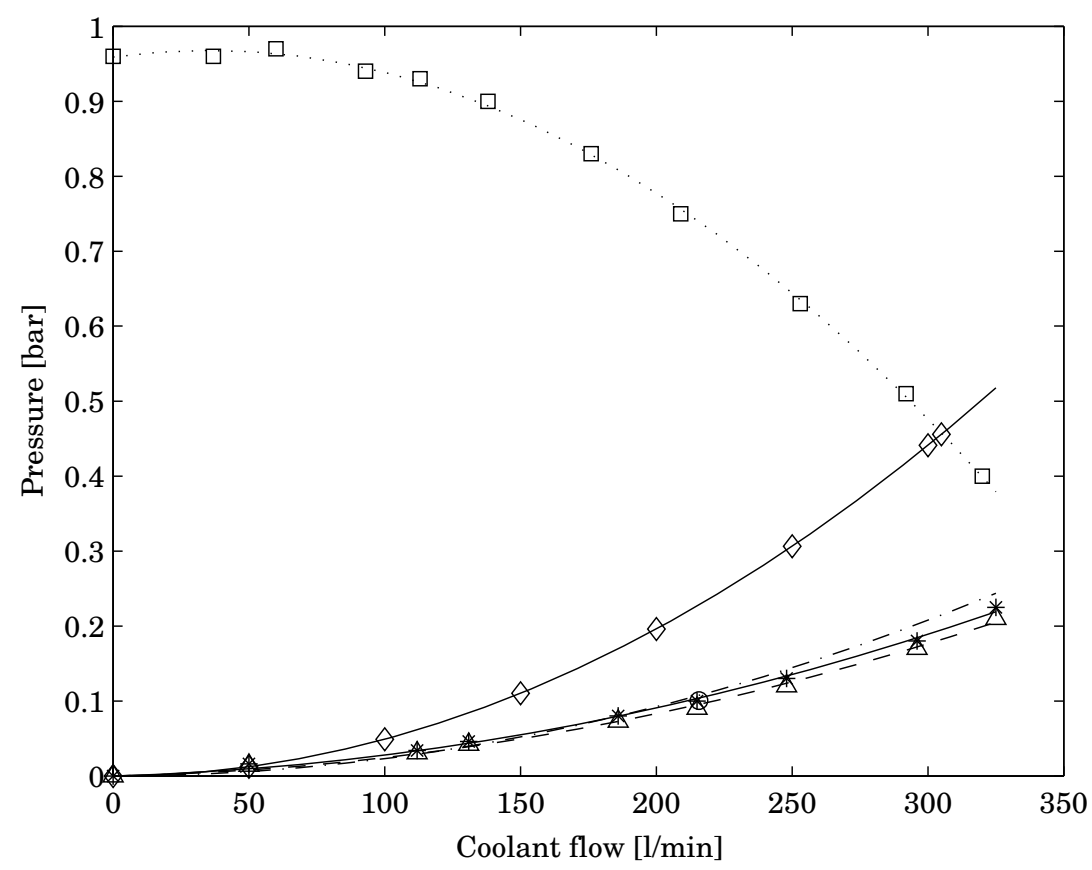

Figure 3.11: Pressure drop as a function of coolant flow for the engine model (solid) compared with measurements (stars). Corresponding drops for the retarder (dashed and triangles), the thermostat (dashdotted and circles) and the radiator (solid and diamonds). Pressure rise of the pump model (dotted) at $1400 \mathrm{rpm}$ engine speed compared with measurements (squares).

\subsection{Assembling the Total Model}

The modelling errors in the sub-models are very small. However, when they are assembled to a full model, effects that are not handled in the sub-models may play an important role. It may be effects from the interconnections between the components such as piping in the truck cab. Further, non-linearities may amplify small errors in the sub-models when these are connected and new feedback paths are closed. It can be shown, using a simplified model of the cooling system, that the change of tem- 


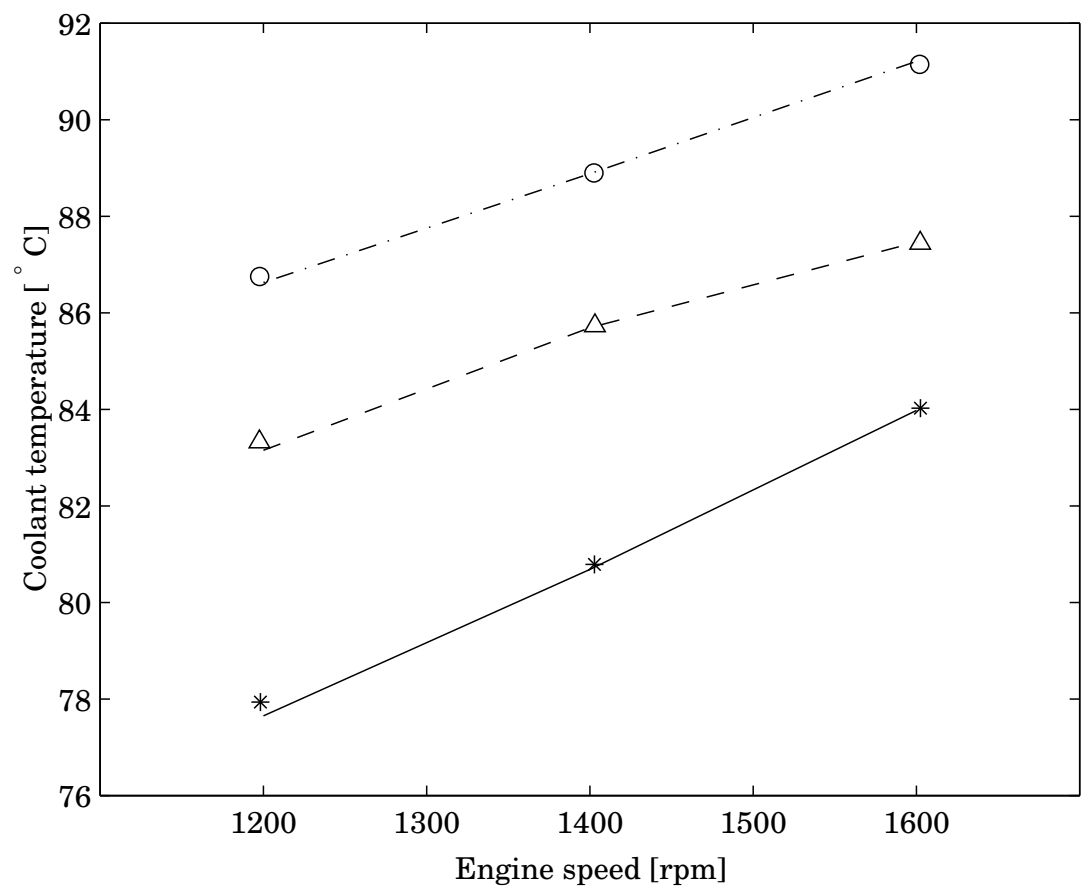

Figure 3.12: Simulated temperature of the coolant in steady state at $80 \mathrm{~km} / \mathrm{h}$ with full load and different speeds on the engine (solid) compared with measurements (stars). Corresponding at $60 \mathrm{~km} / \mathrm{h}$ (dashed and triangles) and at $40 \mathrm{~km} / \mathrm{h}$ (dash-dotted and circles).

perature of the coolant in steady state due to a small perturbation of the airflow is proportional to the squared inverse of the airflow. Thus, the simulated temperature will be very sensitive to modelling errors influencing the airflow. Further, for the pressure build-up due to the wind speed there exists no practicable experiment on a component level. Therefore, the result of the total model is verified through comparison with experimental data collected in a wind tunnel. In the wind tunnel, the vehicle is driven on a dynamometer with a defined load and speed of the engine and fans are used to simulate the wind speed. Results from nine steady-state tests and two step-response tests are used to tune the model parameters. A number of the parameters in the sub-models are assigned 


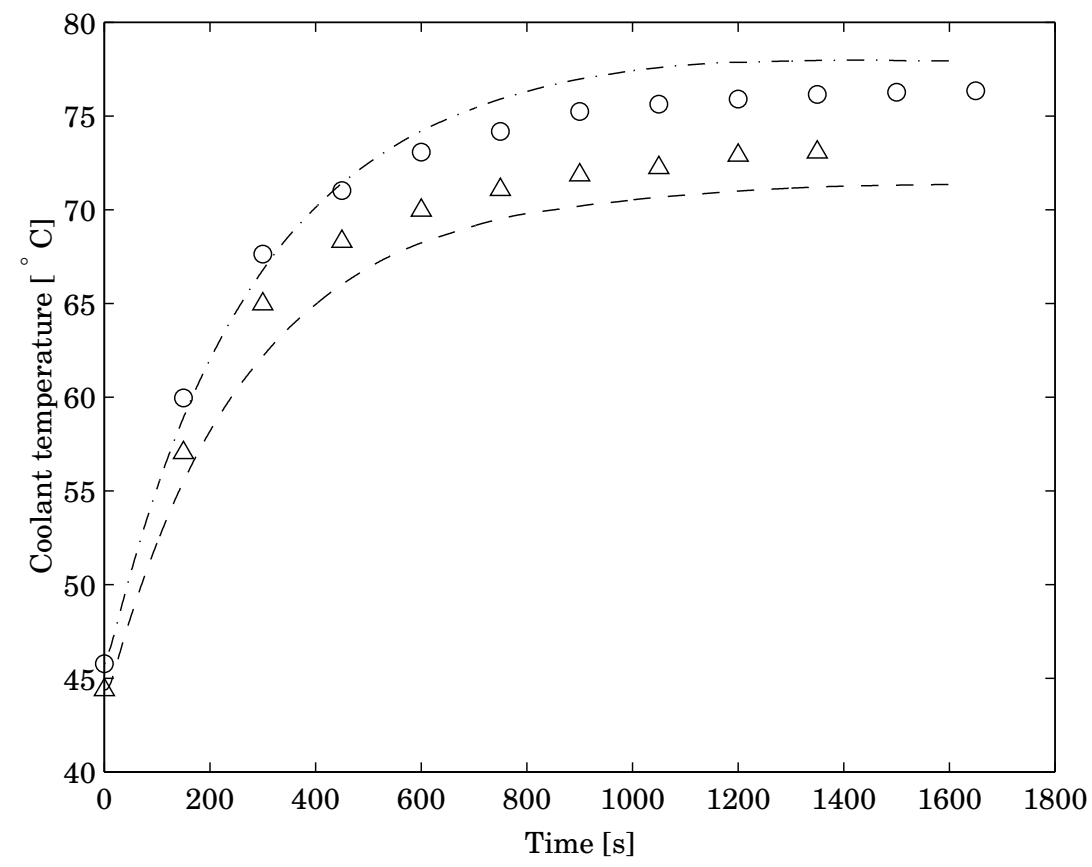

Figure 3.13: Simulated response of the coolant temperature on a step in the engine load at $60 \mathrm{~km} / \mathrm{h}$ with engine speed $1400 \mathrm{rpm}$ (dashed), compared with measurements (triangles). Corresponding at $40 \mathrm{~km} / \mathrm{h}$ (dashdotted and circles).

as slack parameters that are adjusted to fit the behaviour of the total model to the measurements. In table 3.1 the choice of slack parameters is indicated in the last column. The slack parameters are chosen based on engineering practice. Parameters that describe characteristic where it is plausible to expect other phenomena than what could be captured in a test of one component or where no measurement data for the single component is available are preferred as slack parameters. An example of this is selecting the parameter $C D$ in equation (3.3) as slack parameter since this parameter cannot be estimated from a single component test. Another rule is to only use one slack parameter to achieve a certain correction when there are several possible parameters that can be adjusted 


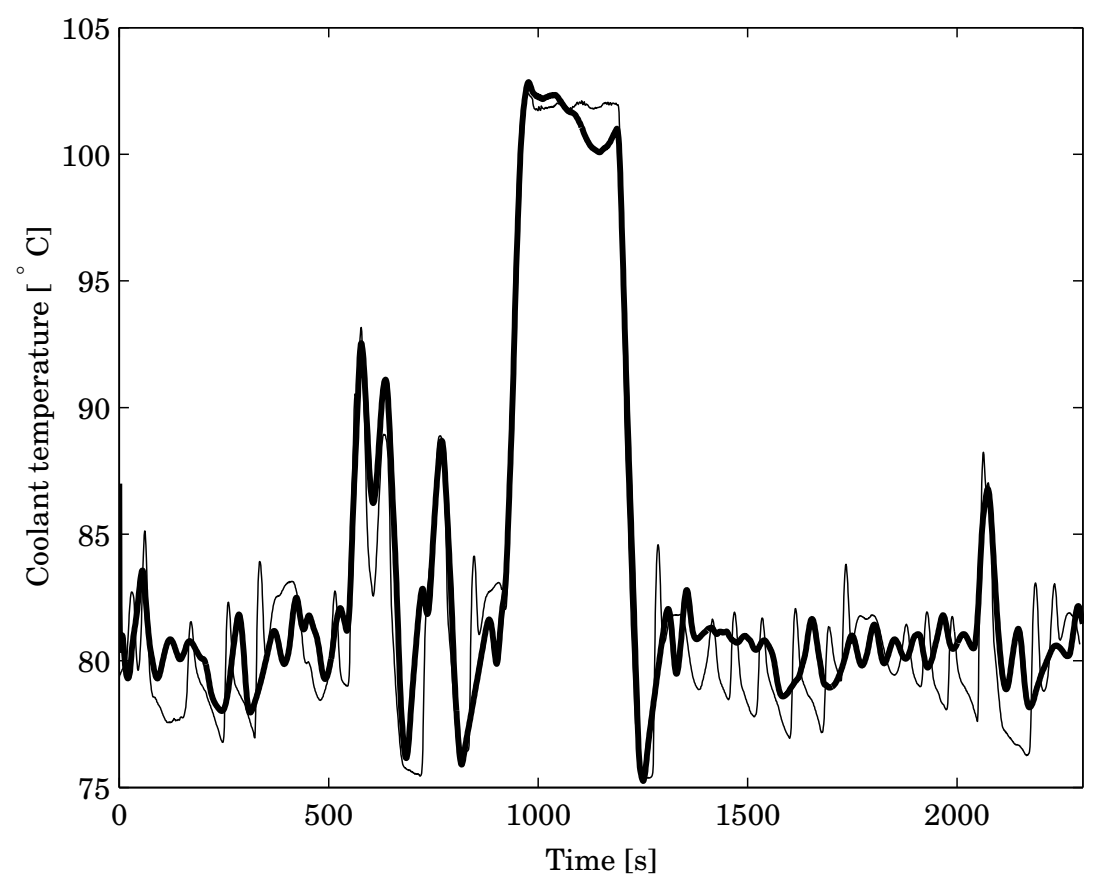

Figure 3.14: Simulated coolant temperature (thick) during a dynamic drive cycle compared with measurements (thin).

to achieve the same effect. An example of the application of this rule is that only the characteristic of the pump is adjusted in order to fit the coolant flow obtained in the simulation to the measured flow on the truck. In figures 3.12 and 3.13 the cooling temperature obtained with the tuned model are compared with measurements. The steady state temperatures obtained with the simulation model agree well with the measurement. The dynamic behaviour in the step-response show good agreement between the model and the measurement but the steady state temperatures differs. As a last step of the parameter identification, a validation of the total model is performed. Validation data is recorded during a dynamic drive cycle in the wind tunnel, where the load and speed of the dynamometer is programmed to follow a cycle corresponding to a specified road. In figure 3.14 the simulation result is compared with measurements 


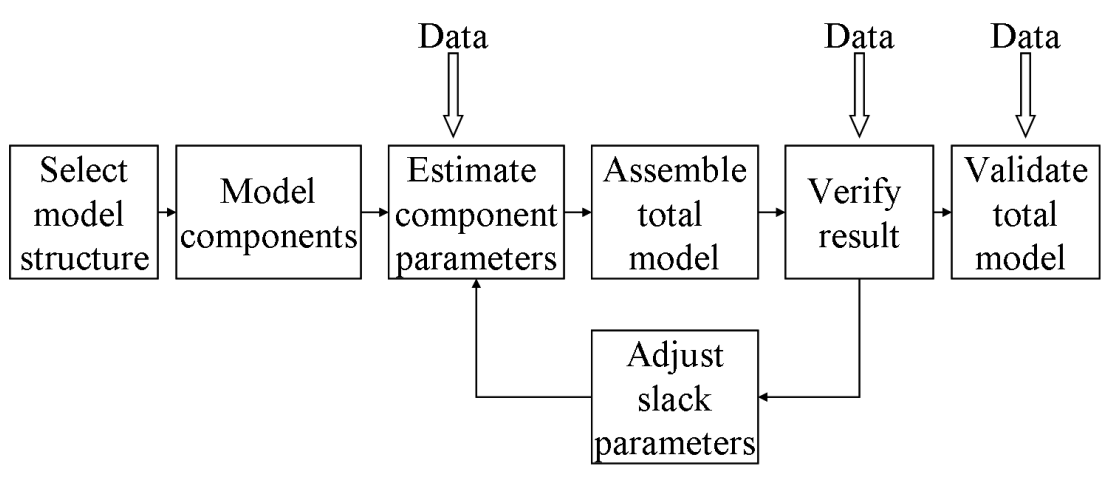

Figure 3.15: Procedure of sequential modelling and identification.

where the dynamometer follows the profile of a $57 \mathrm{~km}$ section of the road between the cities Koblenz and Trier in Germany. The validation shows that the model is capable to capture the main dynamics of the cooling system while it does not describe the small oscillations observed in the measurements. The oscillations around $80^{\circ} \mathrm{C}$ most likely have its origin in the hysteresis in the thermostat due to friction. The model of the thermostat is a rather rough approximation and do not give rise to the corresponding oscillations around the opening temperature. Despite the observed differences, the model should be sufficient to evaluate the energy consumption of the auxiliary units in the cooling system.

\subsection{Summary}

The procedure of building a model consisting of sub-models with physical interpretation and performing sequential identification of parameters may be summarised with the illustration in figure 3.15. Data collected from measurements on single components are used to estimate parameters of the sub-models. The experience from this study is that a good agreement between the simulated behaviour of the sub-models and the measurement do not guarantees accuracy of the total model. Effects not included in the sub-models or small errors in the parameters of the submodels may have a large influence on the model behaviour. Therefore the agreement between the actual system and the simulation output may be 
poor although the sub-model them self seems to be accurate. For that reason, it is necessary to compare output of the complete model with measurements. If a satisfactory result is not obtained, some of the parameters of the sub-models is assigned as slack variables and is adjusted to tune the behaviour of the complete vehicle. When acceptable agreement is obtained, the complete model is validated against new measurements in order to increase the confidence of the model.

The cascaded identification allows for keeping the physical structure of the model while the total behaviour of the model can be tuned to give a good fit to measurement data. As discussed in section 3.1, following the physical structure is advantageous when modelling complex systems in order to be able to include existing control logic, monitor internal variables etc. The drawback with the procedure is of course that a complete installation of the system has to be built in order to be able to collect data that is necessary. However, with suitable choices of interfaces in the model based on physicals principles, the full procedure in figure 3.15 do not necessarily have to apply to the complete model when a sub-model is changed. An important experience from modelling of the cooling system presented here is that the airflow is crucial for the complete model. Minor changes in the airflow result in a rather large change of the obtained cooling temperature. Moreover, the total airflow through the cooling system is practically impossible to measure. Thus, it is not possible to verify that the model gives a correct airflow. Instead the cooling temperature has to be used as an indirect indicator of the airflow. In order to verify the influence from the wind speed, the temperature has to be measured on a rolling vehicle or as shown here in a wind tunnel. The cooling flow on the other hand is straight forward to measure either in a rig or on a complete installation in the truck. These measurements can be done in the laboratory and the measurement setup is typically less complex and costly than the test done in the wind tunnel. If a redesign affecting the coolant flow should be studied with the model, only the sub-models involved in the coolant path have to be changed and validated against measurements. 


\begin{tabular}{|c|c|c|c|}
\hline Component & Characteristic & $\begin{array}{l}\text { Data } \\
\text { source }\end{array}$ & Slack \\
\hline \multirow[t]{2}{*}{ Pump } & Pressure rise & Rig test & $\mathrm{S}$ \\
\hline & Power consumption & Rig test & \\
\hline \multirow[t]{6}{*}{ Engine } & Flow resistance & Rig test & \\
\hline & Heat capacitance & Data sheet & $\mathrm{s}$ \\
\hline & to coolant & Rig test & \\
\hline & Heat conductance & Rig test & \\
\hline & Heat emission & & \\
\hline & from charge air & Rig test & \\
\hline \multirow[t]{4}{*}{ Retarder } & Flow resistance & Rig test & \\
\hline & Heat capacitance & Data sheet & $\mathrm{S}$ \\
\hline & Heat emission & None & \\
\hline & Heat conductance & Rig test & \\
\hline \multirow[t]{3}{*}{ Thermostat } & Opening characteristics & Rig test & \\
\hline & Flow resistance & Rig test & \\
\hline & Dynamic response & Rig test & \\
\hline \multirow[t]{4}{*}{ Radiator } & Flow resistance coolant & Rig test & \\
\hline & Flow resistance air & Rig test & \\
\hline & Operating & Rig test & \\
\hline & Heat capacitance & Data sheet & \\
\hline Air intake & Pressure build-up & None & $\mathrm{S}$ \\
\hline Charge air cooler & Flow resistance & Rig test & $\mathrm{s}$ \\
\hline \multirow[t]{2}{*}{ Fan } & Pressure rise & Rig test & \\
\hline & Power consumption & Rig test & \\
\hline Fan clutch & Slip characteristics & Rig test & \\
\hline Engine & Flow resistance & Rig test & \\
\hline Air outlet & Pressure build-up & None & $\mathrm{s}$ \\
\hline
\end{tabular}

Table 3.1: Summary of model components in the cooling module. 


\section{Chapter 4}

\section{Energy Optimal Control of the Cooling System}

This chapter presents a study on energy optimal control of the cooling system. The configuration of the analysed system is depicted in figure 4.1. The mechanical drives of the water pump and the cooling fan is replaced with electrical drive systems which enable a continuous control of the pump and fan speed. The electrical power required to run the drive systems and other electrical loads is produced with the generator, which is driven by the engine. Further, an electrical buffer enables storage of energy. The controlled quantities are the temperature in the cooling system and the charge level of the electrical energy storage. Control inputs are the speed of the water pump and the cooling fan, and the electrical power of the generator. The objective is to minimise the fuel used to drive the input actuators. This is argued to be comparable with minimising the electrical power of the generator at times when fuel is injected in the engine.

Control strategies like this with the objective to minimise input power could significantly benefits from knowing something about the future external quantities that affect system. For vehicle applications, a possibility to accurately predict the future could be realised with an onboard GPS receiver together with digitalized maps including information of the altitude and speed limits on the travelled road. In the context of this study, the heat emitted to the cooling system, the vehicle speed and the electrical loads are external influences that are not controlled but may be 


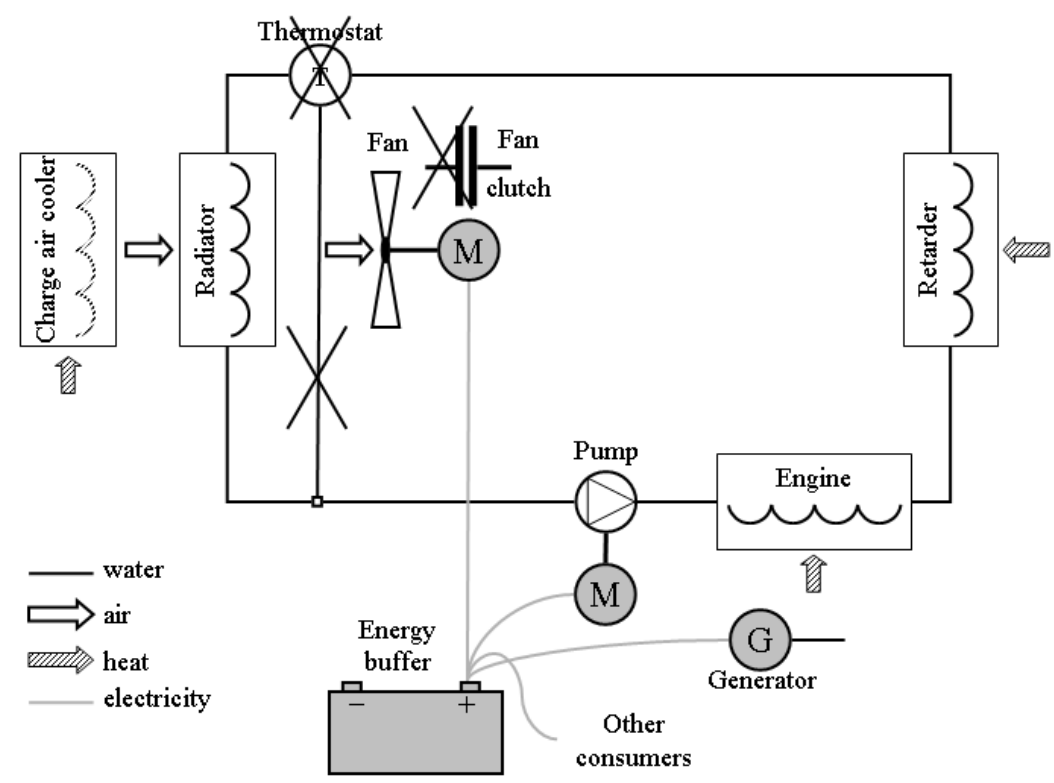

Figure 4.1: Cooling system layout. Components in a traditional truck, but excluded in this study, are $\mathrm{x}$-marked. Novel components added to enable the improved control are grey.

predicted. With a vehicle model, they can be simulated from the slope of the road and the assumed velocity.

The minimisation is posed as a constrained optimal control problem in continuous time where prediction of external variables are utilised. Optimal control theory allows for a wide range of cost criteria and system descriptions. A disadvantage is that solutions are often complicated to find even if the complexity of the system is low. Here a simple, but yet relevant, model is used to describe the physics. The numerical computations for finding the solution of the optimal control problem are reasonable. Our problem formulation fits into the framework of model predictive control, e.g., [Mac02], but the existing schemes seem not to be directly applicable. The solution here is instead based on the particular structures of the dynamics and the cost criterion. The derived optimal control gives a measure of the achievable performance. This is not intended for direct 
implementation but can be used to evaluate different physical layouts.

The studied configuration of the cooling and electrical systems, and the model used for the control design are defined in section 4.1. In section 4.2 the optimal control is derived. Simulation results are presented in section 4.3. Finally in section 4.4, conclusions from the study are drawn.

\subsection{System Configuration}

The principal layout of the cooling system in a truck is described in section 2.5 and is illustrated in figure 2.6. In the current design of a Scania truck, the water pump is directly driven from the crankshaft while the fan is connected to the shaft via a viscous clutch, enabling a passive speed control. The design changes assumed in this study is depicted in figure 4.1. The mechanical drives of the pump and the fan are replaced with speed controllable electrical motors, enabling continuous control of the coolant flow and the airflow. The electricity is produced with a generator driven by the engine. The generator is assumed to be controlled with a power electronic converter. Its overall efficiency is supposed to be substantially higher than in the type of generators used in cars and trucks today. Electric energy can be stored in a buffer, which could be an electro-chemical battery that endures frequent cycling of the charge level and high de-charge and charge peaks. Alternatively, it could be a module of double-layer capacitors (so called super caps or ultra caps), which are inherently well suited for this kind of use. Besides the energy consumption in the pump and the fan, other electrical consumption is regarded. This represents use of electricity for lights, comfort equipment, etc. The thermostat and the by-pass pipe are removed. It is assumed that it is possible to find a minimal coolant flow, which is sufficiently large to prevent the engine from running too hot at the worst spot, while small enough so that the entry temperature to the engine does not fall below the lowest acceptable level. In practice, this might not be true for the whole range of operating conditions. However, the studied concept can with small modification be changed to include an on/off valve acting as a thermostat at low ambient temperatures.

\section{Mathematical model}

In order to apply optimal control theory a mathematical model of the physical system is needed. The Modelica model of the cooling system 
described in chapter 3 contains a fairly detailed description but is too complex to be used for control design. However, it is a valuable starting point for finding a simplified model. The separate volumes and heated masses in the simulation model can be aggregated into one single body representing the total heat capacitance of the cooling system. The single state of the cooling system model is thus the temperature of the aggregated heat capacitance. Let the state, $x_{1}$, represent the temperature in the cooling system minus the ambient temperature. The model is derived from the energy balance in the cooling system: that is, $\dot{x}_{1}$ is proportional to the net power flow into the system. The power flows into the system are the heat transferred to the coolant from the engine and from the retarder. Let $v_{1}$ represent the sum of the heat flows into the system. i.e., this is an external variable that cannot be manipulated. Further, the power flow out of the cooling system is the heat transfer in the radiator. In the simulation model in chapter 3 , the heat transfer in the radiator is factorised into the entry temperature difference and the specific heat transfer, see equation (3.15). Introduce the notation $\Delta \tau$ for the entry temperature difference and $\phi_{s}$ for the specific heat transfer. Then, the model of the cooling temperature can be expressed as

$$
\dot{x}_{1}=-c_{2} \Delta \tau \phi_{s}+c_{1} v_{1}
$$

where $c_{1}$ and $c_{2}$ are scaling constants. The specific heat transfer in the radiator, $\phi_{s}$, describes the nonlinear behaviour of the radiator depicted in figure 3.9. It is a function of the mass flows of coolant and air through the radiator. The coolant mass flow is assumed be proportional to the pump speed, while the air flow is assumed to be proportional to a linear combination of the fan speed and the vehicle speed. The pump speed and the fan speed is represented by the control variables $u_{1}$ and $u_{2}$, respectively, while the vehicle speed is represented by the external variable $v_{3}$. The following low order rational function approximates the behaviour of the radiator depicted in figure 3.9

$$
\phi_{s}=\frac{u_{1}\left(u_{2}+c_{3} v_{3}\right)}{c_{4} u_{1}+u_{1}\left(u_{2}+c_{3} v_{3}\right)+c_{5}\left(u_{2}+c_{3} v_{3}\right)}
$$

Here $c_{3}-c_{5}$ are constant parameters fitted to the behaviour of the cooling system. The entry temperature difference, $\Delta \tau$, equals the temperature of the coolant minus the temperature of the air entering the radiatior. Before entering the radiator, the air is heated up with the heat released in the charge air cooler, see figure 4.1. Since $x_{1}$ represent the coolant temperature above the ambient temperature, the entry temperature difference 


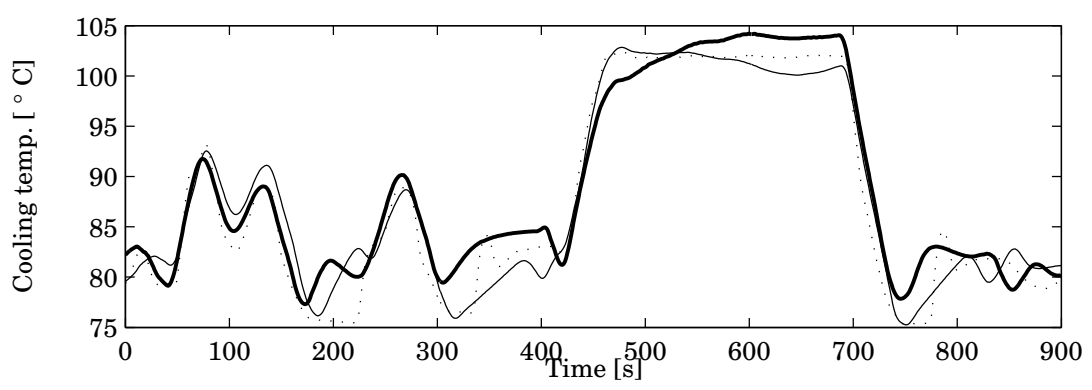

Figure 4.2: Coolant temperature obtained with the model used for control design (thick solid) compared with temperature obtained with the Modelica model (thin solid) and with measurements (dotted).

becomes $x_{1}$ minus the temperature increase in the charge air cooler. The temperature increase is proportional to the heat release divided by the air mass flow. Let the external variable $v_{2}$ represent the heat emission in the charge air cooler. Moreover, according to previous assumptions, the air mass flow is proportional to the linear combination $u_{2}+c_{3} v_{3}$. Thus, the entry temperature difference, $\Delta \tau$, can be expressed as

$$
\Delta \tau=x_{1}-\frac{c_{6} v_{2}}{u_{2}+c_{3} v_{3}}
$$

where $c_{6}$ is a scaling constant. Now equations (4.1)-(4.3) give the following first order model for the cooling system

$$
\begin{aligned}
\dot{x}_{1} & =-c_{2}\left(x_{1}-\frac{c_{6} v_{2}}{u_{2}+c_{3} v_{3}}\right) \frac{u_{1}\left(u_{2}+c_{3} v_{3}\right)}{c_{4} u_{1}+u_{1}\left(u_{2}+c_{3} v_{3}\right)+c_{5}\left(u_{2}+c_{3} v_{3}\right)}+c_{1} v_{1} \\
& \triangleq f_{1}\left(x_{1}, u_{1}, u_{2}, v_{1}, v_{2}, v_{3}\right)
\end{aligned}
$$

A comparison with measurements indicates that this model is able to capture the main dynamics of the cooling system. In figure 4.2 the model in equation (4.4) is compared with the more detailed Modelica model and with the measurement recorded in the wind tunnel.

The energy in the electrical storage is modelled as an integration of the net power into the buffer minus the loss in the buffer. The power loss is assumed to be proportional to the square of the net power. In general, the power loss will be a function of the power flow and the state 


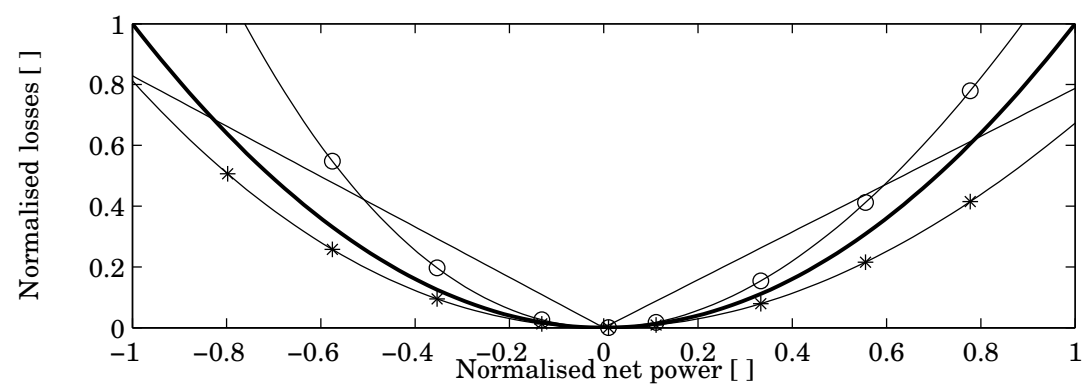

Figure 4.3: Power losses obtained with different models of the energy buffer. The model used for the control design (thick solid) is compared with a constant efficiency model (thin solid), and a circuit model at minimum (solid-circles) and maximum (solid-stars) state of charge.

of charge. When studying the energy level in the storage, a simple model suffices. Related models can be derived from either assuming an efficiency factor of the storage or to model it as an equivalent electrical circuit. In figure 4.3 these different models are compared. The circuit model includes a dependency of the state of charge while the others do not. At low state of charge and a large negative net power, the simpler models tend to underestimate the losses compared to the circuit model. For this study, the quadratic loss model represented by the thick line in figure 4.3 still seams to be sufficient.

Let the state $x_{2}$ represents the state of charge above the lowest allowed level. Further, introduce the notation $\psi_{n}$ for the net power in the terminals of the electrical buffer. Then the model of the electrical system can be expressed as

$$
\dot{x}_{2}=\psi_{n}-c_{7} \psi_{n}^{2}
$$

where $c_{7}$ is a constant parameter describing the efficiency of the buffer. The net power, $\psi_{n}$, is the generated power minus power consumed by the pump, the fan and other electrical equipment. The power produced by the generator is represented by the control variable $u_{3}$. Introduce the notation $\psi_{c}$ for the power consumed by the pump and the fan, and let other electrical consumption be represented with the external variable $v_{4}$. Thus, the net power into the buffer can be expressed as

$$
\psi_{n}=c_{8} u_{3}-c_{9} v_{4}-\psi_{c}
$$


where $c_{8}$ and $c_{9}$ are scaling constants. The power consumption of the pump and the fan, $\psi_{c}$, is described as a cubic function of the pump speed $u_{1}$ and the fan speed $u_{2}$ :

$$
\psi_{c}=c_{10} u_{1}^{3}+c_{11} u_{2}^{3}
$$

Here $c_{10}$ and $c_{11}$ are constant parameters. Now equations (4.5)-(4.7) give the following first order model for the electrical system

$$
\begin{aligned}
\dot{x}_{2} & =c_{8} u_{3}-c_{9} v_{4}-c_{10} u_{1}^{3}-c_{11} u_{2}^{3}-c_{7}\left(c_{8} u_{3}-c_{9} v_{4}-c_{10} u_{1}^{3}-c_{11} u_{2}^{3}\right)^{2} \\
& \triangleq f_{2}\left(u_{1}, u_{2}, u_{3}, v_{4}\right)
\end{aligned}
$$

\subsection{Problem Formulation}

The control objective is to minimise fuel used to drive the generator, while keeping the temperature in the cooling system and the charge level in the energy buffer within specified limits. It is assumed that the time trajectories of $v_{1}, v_{2}, v_{3}$ and $v_{4}$ are known or can be predicted for some time ahead. In practice this prediction horizon is limited by the ability to accurately estimate the external variables from input data. Based on the model defined in the previous section, and knowledge of the future external variables, the optimal input trajectory is calculated. The control objective of minimising the fuel consumption is comparable to minimising the power produced in the generator integrated over times when fuel is injected in the engine, based on the assumption that the both the combustion engine and the generator have a nearly constant efficiency. This is reasonable since the efficiency of the diesel engine in heavy vehicles varies with a few percentage points in the rpm range utilised in highway driving. The generator could be designed with fairly constant efficiency in the corresponding speed interval. The regarded prediction horizon spans from the present, $t_{i}$, up to a fixed time $t_{f}=t_{i}+t_{p}$, where $t_{p}$ is the prediction horizon. Let $\delta=\delta(t)$ be a binary weighting factor that equals one when fuel is needed to drive the vehicle forward, while it is zero when no fuel is injected in the engine. Then, the objective can be formulated as

$$
\min _{u_{1}, u_{2}, u_{3} \in U} J=\int_{t_{i}}^{t_{f}} \delta(t) u_{3}(t) d t
$$

subject to the dynamics (4.4) and (4.8), and the state constraints

$$
0 \leq x_{i_{\min }} \leq x_{i} \leq x_{i_{\max }}=1, i=1,2
$$




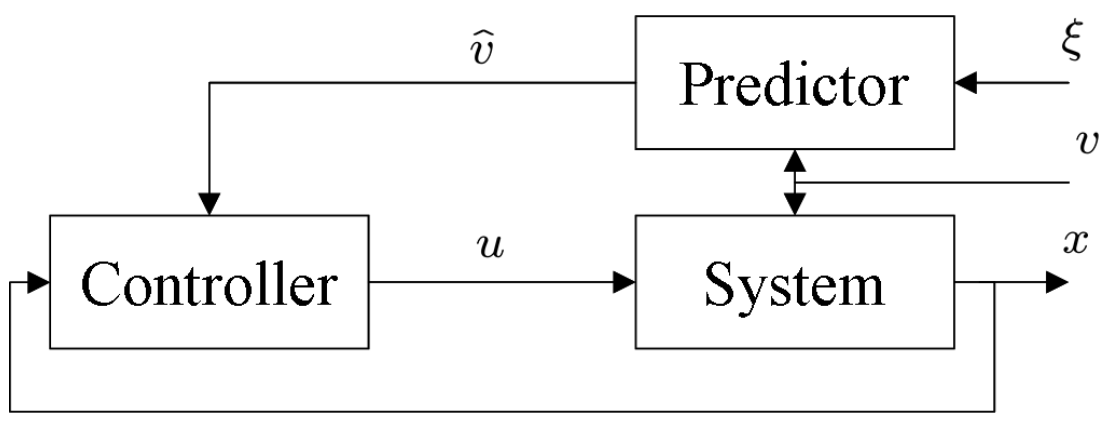

Figure 4.4: Structure of the control system. Feedback of the current state, $x\left(t_{i}\right)$ and feedforward from predictions of the future external influences, $\hat{v}(\tau), \tau \in\left[t_{i}, t_{f}\right]$, are used to derive the optimal control $u(\tau), \tau \in\left[t_{i}, t_{c}\right]$. Input variables to the prediction is the current position of the truck, $\xi\left(t_{i}\right)$, and the current external influences, $v\left(t_{i}\right)$.

The set of admissible controls is equal to

$$
U=\left\{u: 0 \leq u_{i_{\min }} \leq u_{i} \leq 1, i=1,2,3\right\}
$$

The optimisation is performed in a receding horizon scheme where only an initial part of the calculated input is applied. The length of the input trajectory that is applied in each control update is called the control horizon and spans from $t_{i}$ to $t_{i}+t_{c}$, with $t_{c}<t_{p}$. When time $t_{i}+t_{c}$, is reached, the initial and final time is set to $t_{i}:=t_{i}+t_{c}$, and $t_{f}:=t_{f}+t_{c}$, and a new optimal control is derived. In order to force the control not to utilize the buffers in the end of the optimisation interval, constraints on the final states are introduced:

$$
x_{i}\left(t_{f}\right)=\frac{x_{i_{\min }}+x_{i_{\max }}}{2}, i=1,2
$$

The structure of the resulting control system is illustrated in figure 4.4. Feedback of the current state, $x\left(t_{i}\right)$ and feedforward from predictions of the future external influences, $\hat{v}(\tau), \tau \in\left[t_{i}, t_{f}\right]$, are used to derive the control $u(\tau), \tau \in\left[t_{i}, t_{c}\right]$. The prediction of the future influences is based on assumptions of the topology of the road ahead and the velocity. Input variables to the prediction is the current position of the truck, $\xi\left(t_{i}\right)$, and the current external influences, $v\left(t_{i}\right)$. 
The optimal control for the case when state constraints are inactive, is derived from the Hamiltonian [BH75]

$$
H=\delta u_{3}+\lambda_{1} f_{1}\left(x_{1}, u_{1}, u_{2}, v\right)+\lambda_{2} f_{2}(u, v)
$$

where $u$ and $v$ denote the vectors of control and external variables, respectively. The adjoint variables should satisfy the differential equation

$$
\begin{aligned}
& \dot{\lambda}_{1}=-\frac{\partial H}{\partial x_{1}}=-\lambda_{1} \frac{\partial f_{1}}{\partial x_{1}} \\
& \dot{\lambda}_{2}=-\frac{\partial H}{\partial x_{2}}=0
\end{aligned}
$$

Hence $\lambda_{2}$ is constant. In the above setting, the initial and final states are known, while the boundary conditions for the adjoint variables are not. Due to the constraints on the final states, the final values of the adjoint variable, $\lambda\left(t_{f}\right)$, is arbitrary.

\section{Sequential solution}

Suppose first that the state constraints are inactive. The problem above can be numerically solved with a shooting method, [Bry99]. However, although the model involves only two dynamic states, the solution is hard to obtain. The complexity of the problem can be reduced by solving for the control of one state at a time, as discussed next.

Let us first minimise (4.9) with respect to $u_{3}$. For intervals when $\delta(t)=$ 0 , the optimal $u_{3}$, is dependent only on the sign of $\lambda_{2}$. It is straightforward to see that $\lambda_{2}$ should be negative. This gives the control

$$
u_{3}= \begin{cases}\operatorname{sat}^{+}\left(\operatorname{sgn}\left(-\lambda_{2}\right)\right), & \delta=0 \\ \operatorname{sat}^{+}\left(\frac{1+c_{8} \lambda_{2}}{2 c_{7} c_{8}^{2} \lambda_{2}}+\frac{c_{9} v_{4}+\psi_{c}\left(u_{1}, u_{2}\right)}{c_{8}}\right), & \delta=1\end{cases}
$$

where $\operatorname{sat}^{+}(\alpha)=1$, if $\alpha>1,0$ if $\alpha<0$, and $\alpha$ otherwise. Note that when $\delta=1$, not only the sign of $\lambda_{2}$ is of importance. Here $\lambda_{2}$ should be chosen such that the constraints on $x_{2}$ are satisfied. 
If $u_{3}$ does not saturate when $\delta=1$, the Hamiltonian becomes

$$
H=\left\{\begin{array}{cc}
\lambda_{1} f_{1}\left(x_{1}, u_{1}, u_{2}, v\right)+\lambda_{2}\left[c_{8}-c_{9} v_{4}-\psi_{c}\left(u_{1}, u_{2}\right)\right. & \\
\left.-c_{7}\left(c_{8}-c_{9} v_{4}-\psi_{c}\left(u_{1}, u_{2}\right)\right)^{2}\right], & \delta=0 \\
\psi_{c}\left(u_{1}, u_{2}\right) / c_{8}+\lambda_{1} f_{1}\left(x_{1}, u_{1}, u_{2}, v\right)+h\left(\lambda_{2}, v_{4}\right), & \delta=1
\end{array}\right.
$$

where $h\left(\lambda_{2}, v_{4}\right)$ is a known function. If $\delta(t)=1, t_{i} \leq t \leq t_{f}$, the controls $u_{1}$ and $u_{2}$ that minimise $H$ are obviously independent of $u_{3}, \lambda_{2}$ and $x_{2}$. When $\delta(t)=0, t_{i} \leq t \leq t_{f}$, the minimising controls $u_{1}$ and $u_{2}$ are independent of $u_{3}$, and $x_{2}$, but depends on the sign of $\lambda_{2}$ (which is known to be negative). This suggests separation of the control of $x_{1}$ from the control of $x_{2}$ : first $u_{1}, u_{2}$ is derived from (4.16), then $\psi_{c}\left(u_{1}, u_{2}\right)$ is plugged into equation (4.15), giving $u_{3}$. However, if $\delta$ change in the prediction interval, $\lambda_{2}$ must be known when deriving the control of $x_{1}$. The optimal control problem for the cooling system corresponding to the Hamiltonian in (4.16) can thus be formulated as

$$
\begin{aligned}
\min _{u_{1}, u_{2} \in U} J_{c}=\int_{t_{i}}^{t_{f}} & \left\{\delta(t) \psi_{c}\left(u_{1}, u_{2}\right)-(1-\delta) \lambda_{2} c_{8}\left[\psi_{c}\left(u_{1}, u_{2}\right)\right.\right. \\
& \left.\left.+c_{7}\left(c_{8}-c_{9} v_{4}-\psi_{c}\left(u_{1}, u_{2}\right)\right)^{2}\right]\right\} d t
\end{aligned}
$$

subject to the state equations (4.4) and (4.8), and constraints in equation (4.10) and (4.12). Due to the dependence of $\lambda_{2}$ in (4.17) and $u_{1}$, $u_{2}$ in (4.15), the control of $x_{1}$ and $x_{2}$ cannot be separated from each other. However, here the approach is to solve for the control of $x_{1}$ and $x_{2}$ in sequence, and iteratively update the parameters linking the controls together. The simplification this yields is considerable since only onedimensional problems are solved at each step. The iteration scheme and convergence properties of the iteration are discussed in the next section.

\section{Iteration of the sequential solution}

The cooling optimisation problem in equation (4.17) is solved with an initial guess of the constant $\lambda_{2}$. If this value coincides with the solution to equation (4.15), or if $\delta(t) u_{3}(t)=0, t_{i} \leq t \leq t_{f}$, an optimum of the original optimal control problem (4.9) is found. If it does not hold, the sequential solution can be iterated until the optimality condition is satisfied. 
This approach gives the following algorithm:

\section{Algorithm 4.1}

1. Set $\lambda_{2}$ to an initial guess (e.g., $\left.-1 / c_{8}\right)$.

2. Solve the cooling optimisation problem in (4.17) with the current value of $\lambda_{2}$ to obtain $u_{1}$ and $u_{2}$.

3. Find a new $\lambda_{2}$ such that the constraints on $x_{2}$ are satisfied and apply equation (4.15) to obtain $u_{3}$.

4. Terminate if the current $\lambda_{2}$ is close enough to $\lambda_{2}$ used in 1 or if $\delta(t) u_{3}(t)=0, t_{i} \leq t \leq t_{f}$, otherwise jump to 1 .

There are two interesting special values of $\lambda_{2}$ that should be considered when selecting an initial guess. These correspond to a zero cost policy $\left(u_{3}=0\right.$ whenever $\left.\delta=1\right)$ and a constant charge policy $\left(\lambda_{2}=-1 / c_{8}\right.$ giving $\left.f_{2}=0\right)$. With the zero cost policy, the buffer is charged when $\delta=0$, and the stored energy is utilised when $\delta=1$. If it is possible to satisfy the constraints with this policy it will indeed be optimal. With the constant charge policy, the produced power exactly compensate for the consumption. This policy will be optimal whenever $\delta(t)=1, t_{i} \leq t \leq t_{f}$. Solving for the control of a single state implies finding the initial value of the corresponding adjoint variable while the control of the other state is given from the previous iteration. As a measure of the deviation from the correct initial values, one residual is defined for the control of $x_{1}$ and one for the control $x_{2}$. The residuals are evaluated in the end of the time interval where the shooting method is applied and should equal zero when proper initial values are found. The residuals are derived from the conditions at the final time $t_{f}$, or at times when the states entering or leaving the constraints. The optimal control is a function of $x, \lambda$ and $t$. Further, for a given time interval the initial condition of $x$ is fixed. Therefore, the residuals will be functions of the initial condition of $\lambda$ and the end point of the considered time interval. If $t_{s}$ and $t_{e}$ denote start and end point of the time interval, respectively, and the notation $\lambda_{i s} \triangleq \lambda_{i}\left(t_{s}\right), i=1,2$ is introduced, the residuals can be expressed as $r_{i}\left(t_{e}, \lambda_{1 s}, \lambda_{2 s}\right), i=1,2$. Let $r_{1}$ represent condition on the control of $x_{1}$, while $r_{2}$ represent condition on the control of $x_{2}$. In each iteration, $k$, the initial values of $\lambda_{1}$ and $\lambda_{2}$ are updated so that $\lambda_{1 s}^{k+1}$ is the solution of $r_{1}\left(t_{e}, \lambda_{1 s}, \lambda_{2 s}^{k}\right)=0$, while $\lambda_{2 s}^{k+1}$ is the solution of $r_{2}\left(t_{e}, \lambda_{1 s}^{k+1}, \lambda_{2 s}^{k}\right)=0$. Local convergence of 


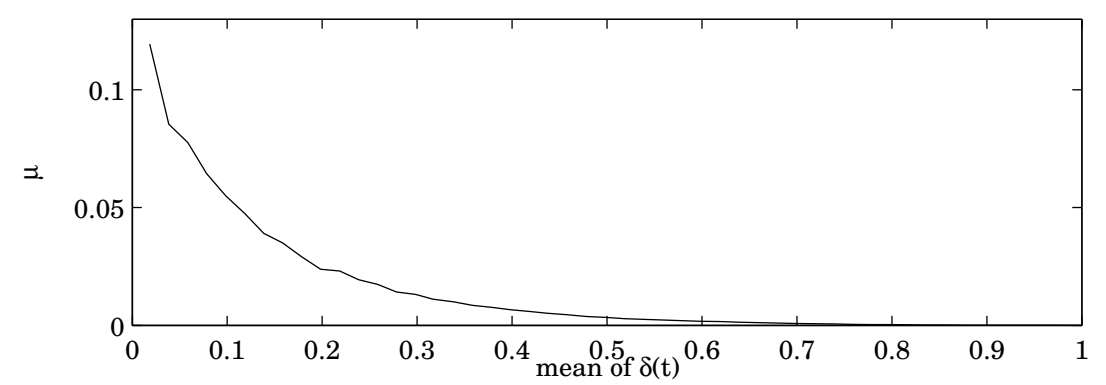

Figure 4.5: Relation (4.18) as a function of the mean of $\delta(t)$

the algorithm 4.1 is obtained if

$$
\mu \triangleq\left|\frac{\partial r_{1}}{\partial \lambda_{2 s}} \frac{\partial r_{2}}{\partial \lambda_{1 s}} /\left(\frac{\partial r_{1}}{\partial \lambda_{1 s}} \frac{\partial r_{2}}{\partial \lambda_{2 s}}\right)\right|<1
$$

holds in a neighbourhood of the optimum. Thus, local convergence is obtained if $\lambda_{1 s}$ mostly influences the control of $x_{1}$, and $\lambda_{2 s}$ mostly influences the control of $x_{2}$. An analytic proof has not been obtained for that relation (4.18) holds when the control does not saturate. Nevertheless, numerical analysis indicates that this is the case. To illustrate this, $\mu$ is evaluated as a function of the mean of $\delta(t)$. The result is shown in figure 4.5. Here the external variables are held constant and constraints are not considered. It exists cases when (4.18) does not holds, for instance when the control inputs saturate over large parts of the prediction horizon. These situations are detected and handled in a special manner.

\section{Handling of state constraints}

The solution to the cooling optimal control problem described in equation (4.17) is straightforward if the state constraints are not encountered (as described above). However, if the state constraints are active the solution is somewhat more complicated, as discussed next. The adjoint variable can be discontinuous at time instances where the state constraints go from being active to non-active, or vice versa. Therefore the optimal trajectory $x_{1}^{*}$ must be divided into constrained and unconstrained arcs. Consider the case when $\delta=1$, and assume that the optimal trajectory consist of the three parts illustrated in figure 4.6: an unconstrained arc, 
$x_{1}^{*}(t), t_{i} \leq t \leq t_{1}$ ending on a constraint, say $x_{1_{\max }}$, a constrained arc, $x_{1}^{*}(t)=x_{1_{\max }}, t_{1} \leq t \leq t_{2}$, ending at $t_{2}$ when the state leaves the constraint, and an unconstrained arc, $x_{1}^{*}(t), t_{2} \leq t \leq t_{f}$ ending at the final state value $x_{1}^{*}\left(t_{f}\right)=\left(x_{1_{\min }}+x_{1_{\max }}\right) / 2$. The optimal control that keeps $x_{1}^{*}(t)=x_{1_{\max }}$ is

$$
\begin{aligned}
{\left[\begin{array}{l}
u_{1} \\
u_{2}
\end{array}\right] } & =\arg \min \left\{\psi_{c}\left(u_{1}, u_{2}\right): u \in U, f_{1}\left(x_{1_{\max }}, u_{1}, u_{2}, v\right)=0\right\} \\
& \triangleq g\left(x_{1_{\max }}, v\right)
\end{aligned}
$$

In this case the cost function can be re-written as

$$
\begin{aligned}
J_{c}= & \int_{t_{i}}^{t_{f}} \psi_{c}\left(u_{1}, u_{2}\right) d t \\
= & \int_{t_{i}}^{t_{1}} \psi_{c}\left(u_{1}, u_{2}\right) d t+\int_{t_{1}}^{t_{2}} \psi_{c}\left(g\left(x_{1_{\max }}\right), v\right) d t+\int_{t_{2}}^{t_{f}} \psi_{c}\left(u_{1}, u_{2}\right) d t \\
= & \underbrace{\int_{t_{i}}^{t_{1}} \psi_{c}\left(u_{1}, u_{2}\right) d t-\int_{t_{i}}^{t_{f}} \psi_{c}\left(g\left(x_{1_{\max }}\right), v\right) d t}_{J_{c_{1}}} \\
& +\underbrace{\int_{t_{2}}^{t_{f}} \psi_{c}\left(u_{1}, u_{2}\right) d t-\int_{t_{2}}^{t_{f}} \psi_{c}\left(g\left(x_{1_{\max }}\right), v\right) d t}_{J_{c_{2}}} \\
& +\underbrace{\int_{t_{i}}^{t_{f}} \psi_{c}\left(g\left(x_{1_{\max }}, v\right)\right) d t}_{J_{c_{0}}}
\end{aligned}
$$

Since $J_{c_{0}}$ is independent of $u, t_{1}$ and $t_{2}$, the problem can be divided into two separate optimal control problems over unconstrained arcs. The times $t_{1}$ and $t_{2}$ are free parameters. Hence, the optimisation should be performed over open time intervals. The condition on the final state (4.12) is now replaced by the condition that the corresponding Hamiltonian should equal zero when the state is entering or leaving the constraint, [BH75]. The discussion above can easily be extended to a general case when the state trajectory enters and leaves the constraint several times. The cost function then becomes

$$
J_{c}=J_{c_{1}}+J_{c_{1}}+\sum_{k=3,5,7, \ldots, N} J_{c_{k}}\left(t_{k}, t_{k+1}\right)+J_{c_{0}}
$$




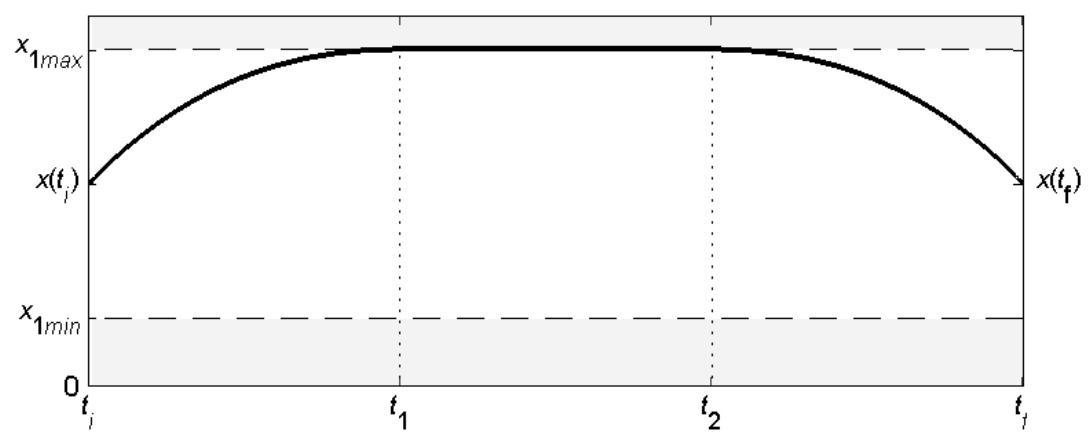

Figure 4.6: The the optimal trajectory $x_{1}^{*}$ is divided into constrained and unconstrained arcs.

Consequently, the optimisation can always be separated into independent problems over unconstrained arcs. In the numerical solution this is done iteratively, searching over the prediction horizon for trajectories that satisfy the condition that the Hamiltonian is equal to zero at the entry and exit to the constraints.

\subsection{Simulation Results}

The optimal control strategy is simulated with input data collected from the experiment in the wind tunnel. The external variables $v_{1}, v_{2}$ and $v_{3}$ are measured on the truck when the load and speed of the dynamometer are programmed to follow trajectories corresponding to a specified road. Simulations are performed over two road sections with altitudes depicted in figure 4.7. The first simulation runs over part of the road between Koblenz and Trier in Germany. The second simulation runs over part of the road between Södertälje and Norrköping in Sweden. These are two $57 \mathrm{~km}$ long sections of the routes used in chapter 2 where the consumption of all the auxiliary units is evaluated. The German route contains rather steep downhill slopes where a lot of heat is emitted to the cooling system from the retarder. The section of the road in Sweden contains long flat sections with some moderate uphill slopes where the engine produces heat that have to be cooled away. The prediction horizon is set to $t_{p}=600$ seconds and the control horizon to $t_{c}=100$ seconds. It is 


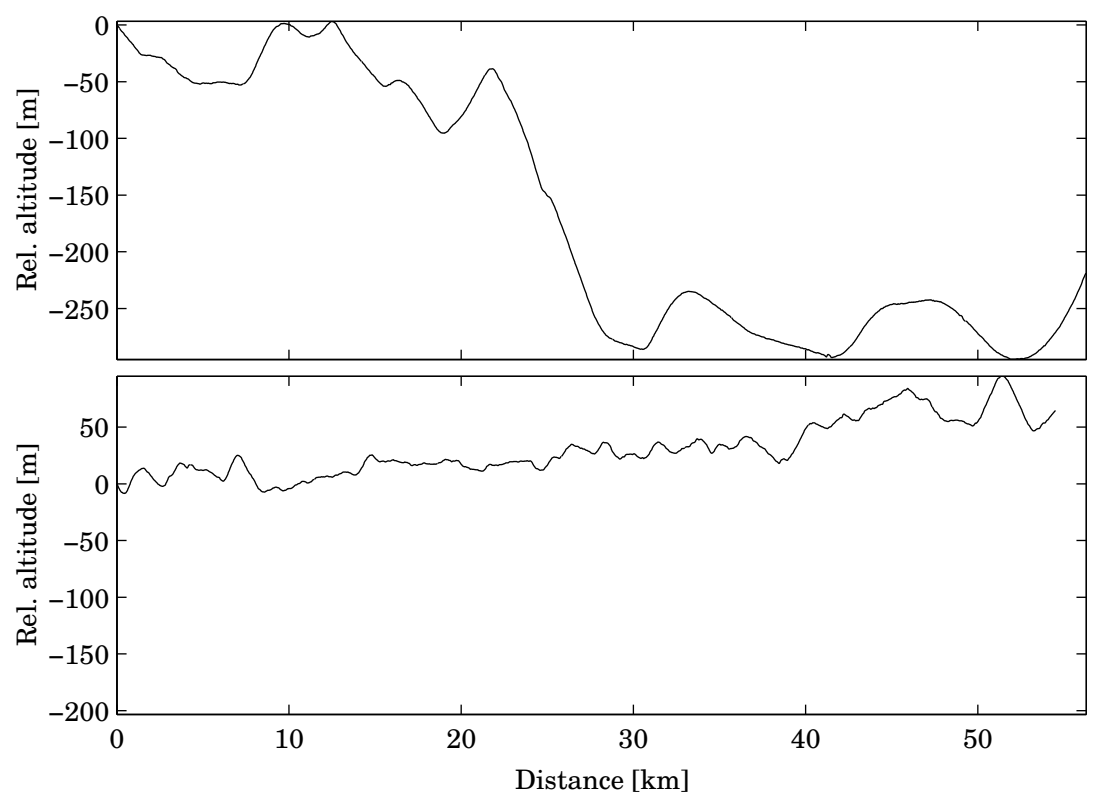

Figure 4.7: Road altitudes used to collect external data. The used part of the Koblenz-Trier road is shown in the upper plot and the used part of the Södertälje-Norrköping road in the lower.

assumed that the controller has exact knowledge of the external variables over the prediction horizon. The parameters of the electrical components are chosen to realistic values. In figures 4.8 and 4.9 , the simulation results are shown. Simulations where optimal control is applied (thick lines) is compared with measurements on a traditional truck (thin lines). In the upper plot, the temperature obtained with optimal control (thick) and measured temperature (thin) is shown. The second plot shows optimal control of the pump (thick solid) and the fan (thick solid-stars) compared with the speed of the pump (thin solid) and the fan (thin solid-stars) in the traditional truck. The third plot shows simulated state of charge in the energy buffer. The forth plot shows optimal control of the generator and indicates the intervals where $\delta(t)=1$ as a bar on the time axis. The lowest plot shows the energy taken from the engine (when $\delta=1$ ), with optimal control (thick) and with traditional control (thin). For the 
optimal control, the plotted energy consumption equals the cost function in (4.9) divided by the efficiency factor of the generator. The energy consumption in the traditional truck is calculated as the sum of the energy consumed in the pump, the fan including the clutch, and the generator. The generator is assumed to have the same efficiency as in the novel system where the optimal control is applied although the existing generator is less efficient. Note that with the optimal control, the energy saving is significant in both figure 4.8 and 4.9. The optimal control utilizes the admissible range of coolant temperature and state of charge. Therefore most of the electricity can be produced when $\delta(t)=0$, i.e., at times $t$ when no fuel is injected in the engine and auxiliary loads can be added without any cost. This can be seen in the forth plot of the figures where the bar on the time axis indicates when $\delta(t)=1$. The variables $\delta$ and $u_{3}$ are simultaneously non-zero only in the interval 800 to $900 \mathrm{~s}$ in figure 4.8 and in the interval 350 to $800 \mathrm{~s}$ in figure 4.9. As a result, the accumulated cost to drive the auxiliary systems, shown in the lowest plot of the figures, increases only in these intervals. All other times, the auxiliaries are run without cost.

Note that neither of the optimal or the traditional control is able to keep the temperature within the required limits in the first simulation (figure 4.8). With the optimal control, the electrical buffer is empty and the generator is saturated when that happens at about $1000 \mathrm{~s}$, and thus, the temperature constraint cannot be satisfied. However it is notable that the optimal control solution prepares for this situation and lowers the temperature as much as possible before this occurs (the temperature is equal to $x_{1_{\min }}=70^{\circ} \mathrm{C}$ at $t=900 \mathrm{~s}$ ). This can be done since the controller know about future external influences.

It should be noticed that the fan clutch installed on the truck in the measurements is of the older type controlled with a bimetal and with relatively high idle speed $(\approx 700 \mathrm{rpm})$. The other type of fan clutch available in the current design of Scania vehicles is electronically controlled and have lower idle speed $(\approx 250 \mathrm{rpm}$ instead of $\approx 700 \mathrm{rpm})$. The two versions of existing fan control is further described in section 2.5. In order to compare the optimal control with a system with the electronically controlled fan, the simulation principle described in chapter 2 is applied. The part of the fuel consumption, $r_{a u x}$, the mean power consumption, $\bar{P}_{a u x}$, and the mean fuel consumption, $\bar{f}_{\text {aux }}$, of the cooling and the electrical system are calculated according to the principles described in chapter 2 . The calculations are performed for the measured operation of the system with the fan controlled by a bimetal, and simulated operation with the 

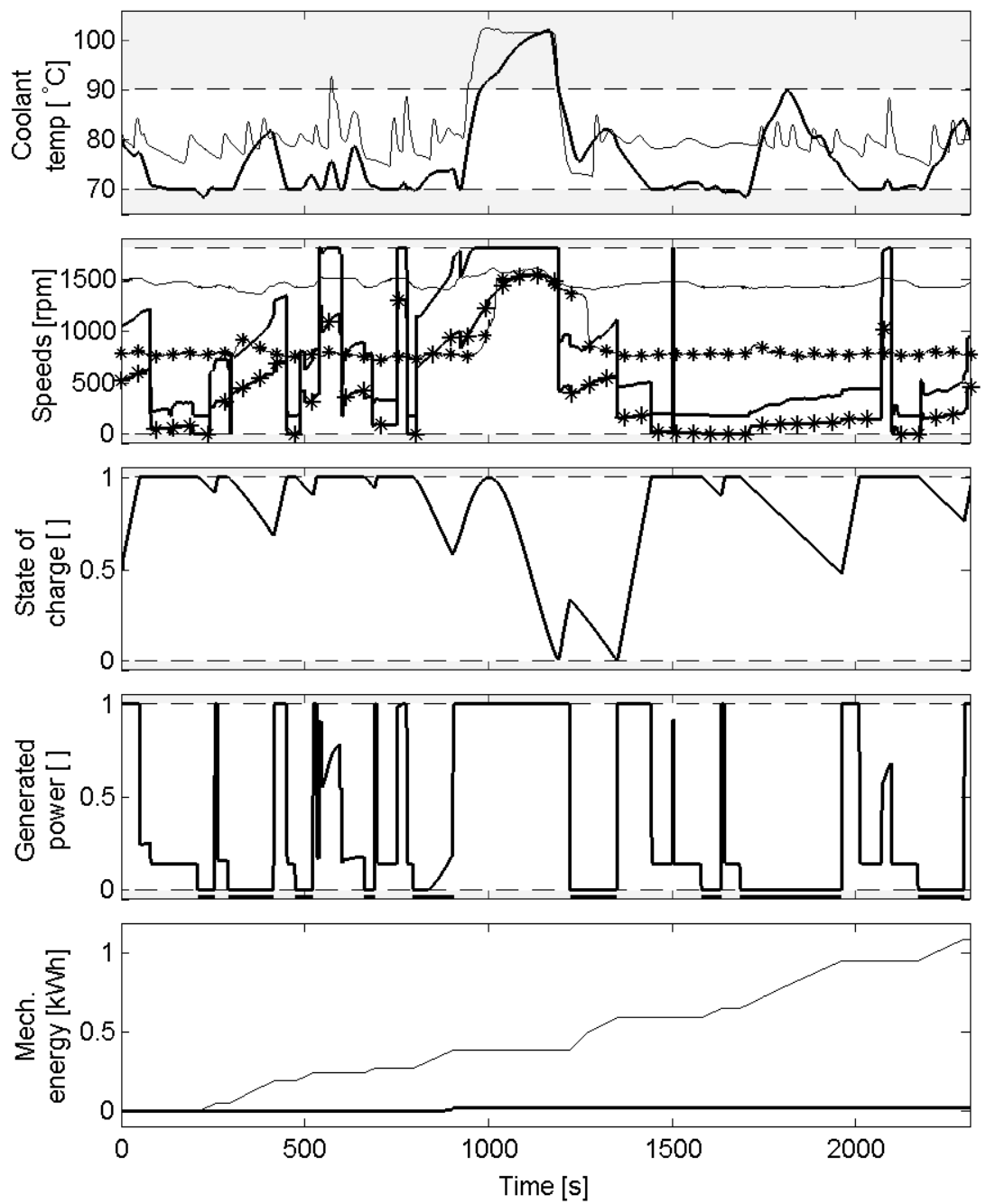

Figure 4.8: Simulation results on the Koblenz-Trier road. The variables are explained in the text. 

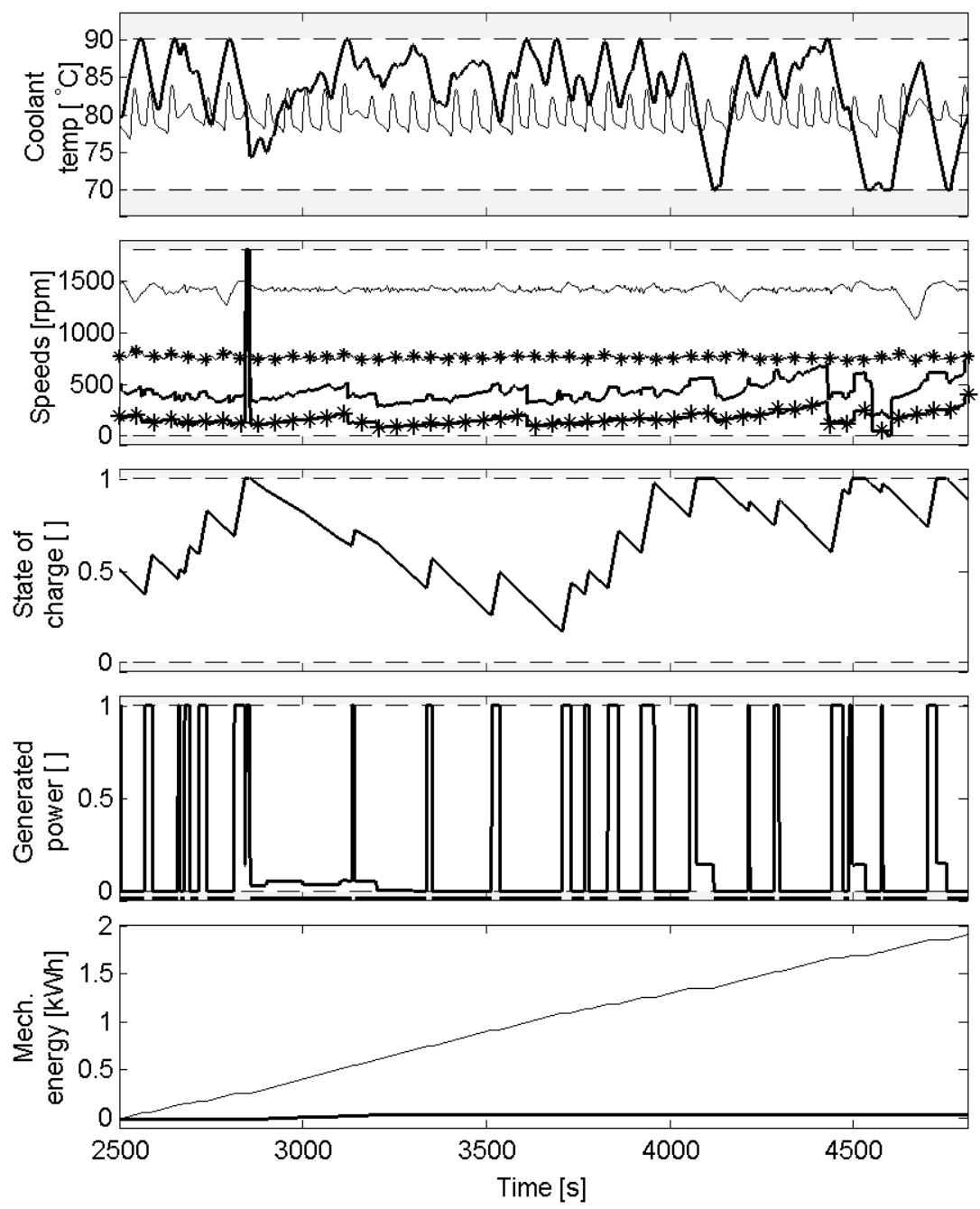

Figure 4.9: Simulation results on the Södertälje-Norrköping road. The variables are explained in the text. 


\begin{tabular}{|c|c|c|c|}
\hline \multicolumn{4}{|c|}{ Koblenz-Trier } \\
\hline Auxiliary & $\begin{array}{l}\bar{P}_{\text {aux }} \\
{[\mathrm{kW}]}\end{array}$ & $\begin{array}{c}\bar{f} \text { aux } \\
{[1 / 100 \mathrm{~km}]}\end{array}$ & $\begin{array}{c}r_{\mathrm{aux}} \\
{[\%]}\end{array}$ \\
\hline Bimetal control of fan clutch & 4.62 & 0.43 & 2.45 \\
\hline Electronic control of fan clutch & 2.85 & 0.20 & 1.13 \\
\hline Optimal control of complete system & 3.40 & 0.01 & 0.07 \\
\hline
\end{tabular}

Table 4.1: Comparison of the consumption for the cooling and electrical system obtained on the Koblenz-Trier route with different control strategies. The columns contain the mean power consumption $\bar{P}_{\text {aux }}$ in $\mathrm{kW}$, the mean fuel consumption $\bar{f}_{\text {aux }}$ in litre $/ 100 \mathrm{~km}$, and the relative fuel consumption $r_{\text {aux }}$ in $\%$.

\begin{tabular}{|c|c|c|c|}
\hline \multicolumn{4}{|c|}{ Södertälje - Norrköping } \\
\hline Auxiliary & $\begin{array}{l}\bar{P}_{\text {aux }} \\
{[\mathrm{kW}]}\end{array}$ & $\begin{array}{c}\bar{f} \text { aux } \\
{[1 / 100 \mathrm{~km}]}\end{array}$ & $\begin{array}{c}r_{\mathrm{aux}} \\
{[\%]} \\
\end{array}$ \\
\hline Bimetal control of fan clutch & 4.62 & 0.43 & 2.45 \\
\hline Electronic control of fan clutch & 2.85 & 0.20 & 1.13 \\
\hline Optimal control of complete system & 3.40 & 0.01 & 0.07 \\
\hline
\end{tabular}

Table 4.2: Comparison of the consumption for the cooling and electrical system obtained on the Södertälje-Norrköping route with different control strategies. The columns contain the mean power consumption $\bar{P}_{\text {aux }}$ in $\mathrm{kW}$, the mean fuel consumption $\bar{f}_{\text {aux }}$ in litre $/ 100 \mathrm{~km}$, and the relative fuel consumption $r_{\text {aux }}$ in $\%$.

electronically controlled fan and the optimal control derived here. The efficiency of the generator is assumed to be the same in all three cases and the trajectories of the vehicle speed, engine torque etc. are taken from the measurement in the wind tunnel. The result is summarised in table 4.1 and 4.2. The calculated mean fuel consumption further illustrate that the optimal control utilise the buffering capacity in the system. Energy is taken from the engine mostly when it does not influence the fuel consumption, i.e., when $\delta=0$. In comparison with the cooling system with electronically controlled fan, the energy saving with the optimal control is lower but still considerable. 


\subsection{Conclusions}

Optimal control of electrically driven auxiliaries in the cooling system of heavy vehicles is derived. The result shows that controllable components in conjunction with prediction of external influences offer a potential to save fuel in this type of application. The saving represents a fraction (1-2\%) of the total fuel consumption, but improvements of this order is certainly worth considering since fuel economy is one of the most important performance factors of long haulage trucks. 


\section{Chapter 5}

\section{Summary and Future Work}

\section{$5.1 \quad$ Summary}

In the thesis the energy consumption of the auxiliary units in heavy vehicles were analysed. Studied auxiliary units were

- Electrical generator.

- Cooling fan.

- Water pump.

- Air compressor.

- Air condition compressor.

- Oil pump.

- Power steering pump.

The potential benefits of driving the auxiliaries electrically were investigated. With electrical drives the output of the auxiliaries can continuously be adjusted to the desired level and losses present in today's mechanical drives can be removed.

The thesis started with an introductory survey over the auxiliary units as they are designed today and how they influence the overall fuel economy of the vehicle. The simulation indicated that the total part of the fuel consumption that can be derived from the considered auxiliary units is in the range of $4.7 \%$ to $7.3 \%$. The performed survey gives an upper 
limit on what fuel saving that can be achieved if the auxiliary units are redesigned. Clearly one cannot expect to gain more than part of the total fuel consumption of the current systems. The study illustrates the relation between the energy consumption of the auxiliary systems.

A model library, which can be used to evaluate novel drive systems and control principles for the auxiliary units, was developed in the modelling language Modelica. The library contains a mixture of models developed from physical principles and models fitted to collected data. Modelling of the cooling system was described in some detail. Simulation results are compared with measurement data from tests in a wind tunnel.

A case study on energy optimal control of the cooling and the electrical system was presented. Optimal control theory was employed to derive the control of the electrical generator, and the water pump and the cooling fan, which both were supposed to be electrically driven. The optimal controller gave a significant energy saving. The assumptions on the electrical components are preliminary, in order to give more precise estimates of the achievable energy saving, refined models need to be derived in future studies.

\subsection{Future Work}

In this section some proposals for future work are discussed. The discussion is organised according to the chapters in the thesis.

\section{Evaluating energy consumption}

The simulations performed in chapter 2 gives estimate on the fuel consumption caused by the auxiliary units. In order to increase the accuracy of the estimation, some of assumptions that define the operation of the auxiliaries need to be further verified with measurements. For instance, more statistics on the operation of the auxiliaries could be gathered from trucks running in daily operation. Further, the definition of the drive cycles of the vehicle should be extended to include engine idling. Statistics collected in long-haulage trucks in field operation indicates that the idling time constitutes a non-negligible part of the total operating time, up to five to ten percents. An adequate amount of idling time should be included in the drive cycles used in future investigations in order to obtain results that better reflect the average consumption of the auxiliaries in real-life operation. If idle time is included, the relative consumption of the generator and the air conditioning compressor are likely to increase. 


\section{Modelling energy consumption}

The model library presented in 3 should be extended to include modelling of all of the auxiliary systems. The work with the simplified models in chapter 2 can serve as a good starting point when developing models in Modelica for the library. Simultaneously, there is a need to incorporate less a complex model of the cooling system in the Modelica library. The presented model in is computational expensive in relation to the rest of the vehicle model. The level of detail included in the model may not be needed in simulations where only the total effect of the on the fuel consumption is of interest. For this purposes, a model like the cooling model used in chapter 2 is preferable. On the other hand, for specific studies of the cooling system, the model in chapter 3 is not detailed enough. For instance, effects from exhaust gas recirculation (EGR) are not explicitly handled. Hence, it is desired that the library could be extended with models with various level of detail.

\section{Deriving energy optimal control}

The optimal control derived in chapter 4 gives an indication on achievable performance, but is not intended for direct implementation. Further work on controller design and prediction of the future influences are needed in order to implement a similar scheme in the vehicle.

To be able to implement an energy optimal control of the auxiliary units it is important to consider the constraints imposed by the available hardware in vehicles. It is not foreseen in a near future that a true optimal control strategy can be implemented in the vehicle. Therefore suboptimal strategies have to be considered. One possible strategy includes simplifying the system dynamics with a time discrete and piecewise affine system, see e.g. [BABH00]. In this setting it is possible to derive an explicit solution. However, the explicit solution is not practicable with a prediction horizon of any considerable length. To solve the mixed integer problem resulting from this problem formulation online is probable not possible either. Another possible way to proceed could be to apply dynamic programming, see [FB04]. Unfortunately, discrete dynamic programming does not seem to scale very well to larger systems with several states. Perhaps controllers of traditional structure can be tuned to achieve performance close to the optimum. An issue to address in future work is then how predictions of the future can be incorporated in such a structure.

Prediction of external influences used in the control scheme can on 
principle be realised as outlined in chapter 4: GPS receivers are becoming common in vehicles as they are utilised in navigation and anti-theft systems. With the navigation systems follows digitalised maps covering large parts of the road net. Prediction of the external influences can in be obtained if a vehicle model is simulated on the road ahead. However, how to get an acceptable accuracy of the predictions might not be straightforward. Future work is needed to analyse accuracy and implementation issues of prediction algorithms. 


\section{Bibliography}

[BABH00] F. Borrelli, M. Fodor A. Bemporad, and D. Hrovat. A hybrid approach to traction control. In Lecture Notes in Computer Science, chapter Hybrid Systems: Computation and Control. Springer Verlag, 2000.

[Ben04] P. Bengtsson. Structuring of models intended for complete vehicle simulation. Master's thesis, Uppsala University, 2004.

[BH75] A. E. Bryson and Y. C. Ho. Applied optimal control. Hemisphere Publishing Company, Washington D.C., 1975.

$\left[\mathrm{BHH}^{+} 02\right]$ A. Brooker, K. Haraldsson, T. Hendricks, V. Johnson, K. Kelly, B. Kramer, T. Markel, M. O'Keefe, S. Sprik, K. Wipke, and M. Zolot. Advisor documentation. Technical report, NREL, 2002.

[BMDP02] A. Bemporad, M. Morari, V. Dua, and E.N. Pistikopoulos. The explicit linear quadratic regulator for constrained systems. Automatica, 38(1):3-20, 2002.

[Bry99] A. E. Bryson. Dynamic Optimization. Addison Wesley Longman Inc., Menlo Park, CA., 1999.

[BTK04] M. Back, S. Terwen, and V. Krebs. Predictive powertrain control for hybrid electrical vehicles. In Proceedings of $1 s t$ IFAC Symposium on Advances in Automotive Control, 2004.

[DOE00] U.S. Department Of Energy. Technology roadmap for the 21st century truck program. Technical report, DOE, 2000.

[FB04] E. Finkeldei and M. Back. Implementing a MPC algorithm in a vehicle with a hybrid powertrain using telematics as sensor 
for powertrain control. In Proceedings of 1st IFAC Symposium on Advances in Automotive Control, 2004.

[HLBG00] W. Hnatczuk, M. P. Lasecki, J. Bishop, and J. Goodell. Parasitic loss reduction for 21st century trucks. SAE Paper 200001-3423, 2000.

[HO02] T. Hendricks and M. O'Keefe. Heavy vehicle auxiliary load electrification for the essential power system program: Benefits, tradeoffs, and remaining challenges. SAE Paper 200201-3125, 2002.

[LA03] L. Laine and J. Andreasson. Modelling of generic hybrid electric vehicles. In Proceedings of the 3rd International Modelica Conference, 2003.

[LC03] M. P. Lasecki and J. M. Cousineau. Controllable electric oil pumps in heavy duty diesel engines. SAE Paper 2003-01-3421, 2003.

[Lju99] Lennart Ljung. System Identification - Theory For the User, 2nd ed. PTR Prentice Hall, Upper Saddle River, N.J, 1999.

[Mac02] J.M Maciejowski. Predictive control with constraints. Pearson Education Limited, Essex, 2002.

[Mod02] Modelica Association. Modelica - A Unified Object-Oriented Language for Physical Systems Modeling. Language Specification Ver. 2.0. Modelica, 2002. http://www.modelica.org.

[OHLB02] M. O'Keefe, T. Hendricks, J. Lustbader, and A. Brooker. Enhancements to NREL system analysis tools to improve auxiliary load modeling and air conditioner modeling for heavy vehicles. Technical report, NREL, 2002.

[Pet02] R. Pettersson. Evaluation of energy savings in the truck engine achieved with control of the cooling flow, Utvärdering av effektbesparing i lastbilsmotorn vid införande av ett styrbart kylvattenflöde. Master's thesis, Chalmers, 2002. (In Swedish).

[PG84] R. H. Perry and D. Green. Perry's Chemical Engineers' Handbook, sixth edition. McGraw-Hill Book Company, New York, 1984. 
[San01] T. Sandberg. Heavy Truck Modeling for Fuel Consumption Simulation and Measurements. Licentiate thesis, Linköpings Universitet, 2001.

[SBG04] A. Sciarretta, M. Back, and L. Guzzella. Optimal control of parallel hybrid electric vehicles. IEEE Transaction on control systems technology, 12(3):352-363, May 2004.

[Ste04] Magna Steyr. Simulation of cooling systems, Kuli 4.0. Magna Steyr, 2004. http://wwww.kuli.at.

[TBD03] M. Tiller, P. Bowles, and M. Dempsey. Development of a vehicle modeling architecture in modelica. In Proceedings of the 3rd International Modelica Conference, 2003.

[TEW00] H. Tummescheit, J. Eborn, and F. J. Wagner. Development of a modelica base library for modeling of thermohydraulic systems. In Proceedings of Modelica Workshop 2000, 2000.

[Tum02] H. Tummescheit. Design and Implementation of ObjectOriented Model Libraries using Modelica. PhD thesis, Lund Institute of Technology, 2002.

[VDI94] VDI. VDI-Wärmeatlas: Berechnungsblätter für den Wärmeübergang, 7.Auflage. VDI-Verlag, Düsseldorf, 1994.

[WCB99] K.B. Wipke, M.R. Cuddy, and S.D. Burch. Advisor 2.1: A user-friendly advanced powertrain simulation using a combined backward/forward approach. Technical report, NREL, 1999. 